\title{
Silica-Supported Sodium with Ionic Liquid: A Neutral Catalyst System for Michael Reactions of Indoles in Water
}

\author{
Yanlong Gu, Chikako Ogawa, and Shū Kobayashi* \\ Graduate School of Pharmaceutical Sciences, The University of Tokyo, The HFRE \\ Division, ERATO, Japan Science and Technology Agency (JST), Hongo, Bunkyo-ku, \\ Tokyo 113-0033, Japan
}

\section{Supporting Information}

\section{Experimental}

General. $\quad{ }^{1} \mathrm{H}$ and ${ }^{13} \mathrm{C}$ NMR spectra were recorded on a JEOL JNM-LA300, JNM-LA400, or ECX-400 spectrometer in $\mathrm{CDCl}_{3}$, and tetramethylsilane (TMS) was used as internal standard. IR spectra were measured on a JASCO FT/IR-610 spectrometer. Commercially available chemicals and dry solvents (ethyl acetate, hexane, toluene, THF, and ethanol), purchased from Aldrich, Kanto Chemical, Tokyo Chemical Industry, and Wako Pure Chemical Industry, were purified according to standard procedures before use. Column chromatography was conducted on Silica gel 60 (Merck) and preparative thin-layer chromatography (PTLC) was carried out using Wakogel B-5F. ICP analysis was performed using Shimadzu ICPS-7510. The structures of known compounds were confirmed by comparison with data shown in literature.

Preparation of silica gel-supported sodium. Preparation of silica-supported sodium catalysts was performed according to Scheme S-1. In this work, silica gel with large pore from Strem Chemicals Company was used. As shown in Scheme S-1, arylsulfonic acid-functionalized silica gel (Silica- $\mathrm{PhSO}_{3} \mathrm{H}$ ) was prepared according to a modified synthetic procedure using (chlorosulfonylphenyl) ethyltriethoxysilane (CSPTMS) as a precursor. ${ }^{1-3}$ The detailed procedure is as follows: silica gel was activated before use over $2 \mathrm{~N} \mathrm{HCl}$ at $80{ }^{\circ} \mathrm{C}$ for $8 \mathrm{~h}$. The obtained activated silica was filtrated off and washed with deionized water until the $\mathrm{pH}$ value became 7 . After drying at air and reduced pressure, the activated silica gel $(6.0 \mathrm{~g})$ was mixed with a 
dichloromethane solution of CSPTMS (5 g, $50 \mathrm{wt} \%$ in $\mathrm{CH}_{2} \mathrm{Cl}_{2}$ ) in a $100 \mathrm{~mL}$ round-bottom flask under Ar. Dried toluene was then added, and the mixture was refluxed at $130{ }^{\circ} \mathrm{C}$ for $24 \mathrm{~h}$. After the reaction was complete, the reaction mixture was cooled to room temperature. The solid was filtrated off, and washed with dried toluene $(20 \mathrm{~mL} \times 5$ times $)$. The obtained silica gel material was then dried at $80{ }^{\circ} \mathrm{C}$ for $6 \mathrm{~h}$ under reduced pressure $(1 \mathrm{mmHg})$. This silica gel was then treated with $2 \mathrm{~N} \mathrm{H}_{2} \mathrm{SO}_{4}(50 \mathrm{~mL})$ for $12 \mathrm{~h}$ at $80{ }^{\circ} \mathrm{C}$. After filtration, the obtained solid material was carefully washed with pure water to adjust the $\mathrm{pH}$ value of the solution to 6-7. After drying, ion exchange capacity was determined by a known method. ${ }^{1}$
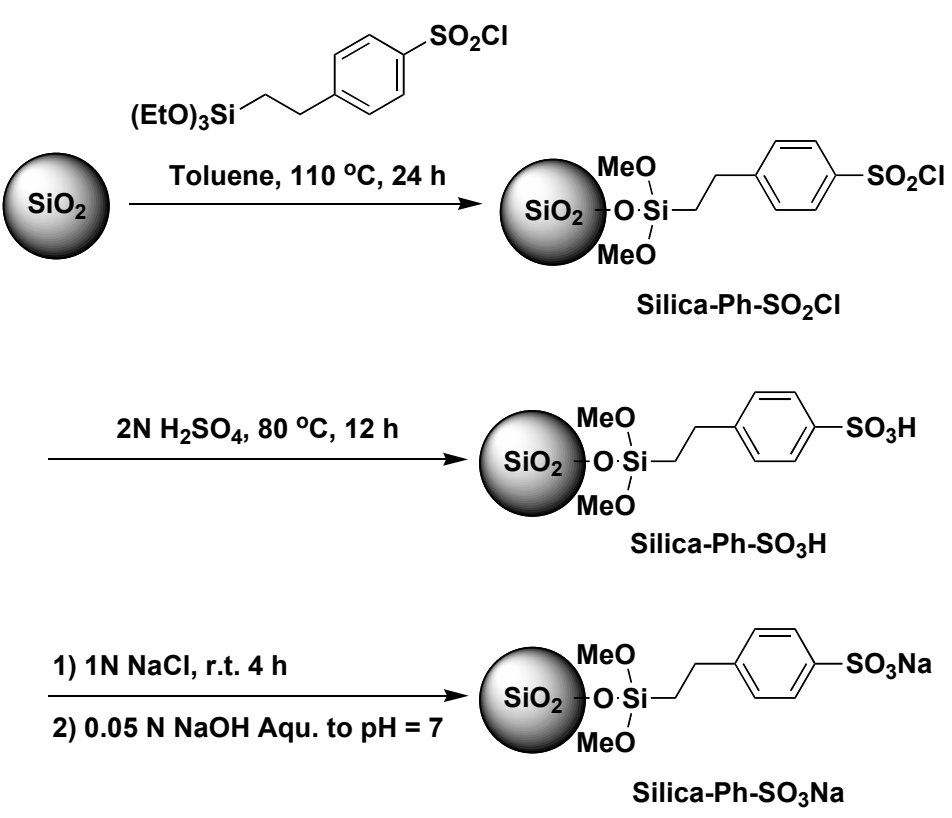

Scheme S-1. Preparation of Silica-Na materials

As a typical procedure, the Silica- $\mathrm{PhSO}_{3} \mathrm{H}$ material $(15.0 \mathrm{mg})$ was mixed with a $\mathrm{NaCl}$ aqueous solution $(20 \mathrm{~mL}, 0.1 \mathrm{~N})$ in a $30 \mathrm{~mL}$ flask, and the mixture was stirred for $24 \mathrm{~h}$ at room temperature. The solution was then titrated by a $\mathrm{NaOH}$ aqueous solution $(0.01 \mathrm{~N})$ to adjust the $\mathrm{PH}$ value to 7 . From the consumption difference of the $\mathrm{NaOH}$ aqueous solution between the activated silica gel and the Silica- $\mathrm{PhSO}_{3} \mathrm{H}$ material, the ion exchange capacity of Silica- $\mathrm{PhSO}_{3} \mathrm{H}$ can be calculated. The obtained Silica- $\mathrm{PhSO}_{3} \mathrm{H}$ material has $0.70 \mathrm{mmolH}^{+} / \mathrm{g}$ of ion exchange capacity. Elemental analysis revealed that the sulfur content is $2.4 \mathrm{wt} \%$ in Silica- $\mathrm{PhSO}_{3} \mathrm{H}$. This is good agreement with the ion exchange capacity. Silica- $\mathrm{PhSO}_{3} \mathrm{H}$ was then converted to the corresponding sodium salt using an aqueous solution of $\mathrm{NaCl}(1.0 \mathrm{~N})$. For complete exchange of Silica- $\mathrm{PhSO}_{3} \mathrm{H}$ to the corresponding sodium salt, an aqueous solution of $\mathrm{NaOH}(0.05 \mathrm{~N})$ was used to neutralize the 
produced $\mathrm{H}^{+}$during the ion exchange procedure. ${ }^{1}$ For decreases of the Na loading, decreased amounts of CSPTMS ( $3 \mathrm{~g}$ and $1 \mathrm{~g}$ ) were used under the same conditions; thus, two Silica-Na catalysts with 0.57 and $0.35 \mathrm{mmol} / \mathrm{g}$ of $\mathrm{Na}$ loadings were obtained.

A typical procedure for Michael reaction of 1-methylindole. All reactions were conducted in a $10 \mathrm{~mL}$ V-type flask equipped with magnetic stirring. In a typical reaction, Silica-Na 3 (Na loading $0.70 \mathrm{mmol} / \mathrm{g}, 50 \mathrm{mg}, 14 \mathrm{~mol} \% \mathrm{Na}$ ) was mixed with $0.25 \mathrm{~mL}$ of the ethyl acetate solution of ionic liquid $[\mathrm{DBIm}] \mathrm{SbF}_{6}\left(12.5 \mathrm{mg}\right.$ of $[\mathrm{DBIm}] \mathrm{SbF}_{6}$ was contained, $25 \mathrm{wt} \%$ with regard to Silica-Na). After a few minuets stirring, ethyl acetate was evaporated and the residue solid material was dried under reduced pressure $(1.0 \mathrm{mmHg})$ for 10 minutes at room temperature. Water (0.25 mL), 1-methylindole (32.8 mg, $0.25 \mathrm{mmol}$ ), and methyl vinyl ketone (MVK, $17.5 \mathrm{mg} 0.25$ mmol) were then introduced successively. The mixture was stirred for $12 \mathrm{~h}$ at $30{ }^{\circ} \mathrm{C}$. After the reaction, the mixture was extracted with a mixture of ethyl acetate and hexane $(1: 1 \mathrm{v} / \mathrm{v}, 6 \mathrm{~mL} \times 4)$. After concentration of the organic phase, the crude compounds were purified by PTLC to give 4-(1-methyl-3-indolyl)-2-butanone (47.8 mg, 95\%). The residue solid was treated under reduced pressure to remove all the volatile components, and then reused in the next run.

In the case of a solid substrate, the silica material, ionic liquid, the solid substrate, and stirring bar were premixed in ethyl acetate before the reaction. After a few minutes stirring, ethyl acetate was evaporated, and the residue solid material was dried under reduced pressure $(1 \mathrm{mmHg})$ at room temperature. Finally, water and a liquid substrate were introduced successively. The following procedure is analogous to that of liquid substrates.

4-(1-Methyl-3-indolyl)-2-butanone (1): ${ }^{4}$<smiles>CC(=O)CCc1cn(C)c2ccccc12</smiles>

(oil, Hexane/EtOAc = 4:1, 87\%); ${ }^{1} \mathrm{H}$ NMR $\delta 2.05,(s, 3 \mathrm{H}), 2.74(t, 2 \mathrm{H}, J=7.3 \mathrm{~Hz}), 2.95(t, 2 \mathrm{H}, J=$ $7.3 \mathrm{~Hz}), 3.63(s, 3 \mathrm{H}), 6.75(s, 1 \mathrm{H}), 7.03(t, 1 \mathrm{H}, J=11.5 \mathrm{~Hz}), 7.12-7.21(m, 2 \mathrm{H}), 7.49,(d, 1 \mathrm{H}, J=$ $7.8 \mathrm{~Hz}) ;{ }^{13} \mathrm{C}$ NMR $\delta 19.2,30.0,32.5,44.3,109.2,113.6,118.6,118.7,121.5,126.3,127.5,136.9$, 208.7 . 


\section{4-(5-Bromo-3-indolyl)-2-butanone (2): ${ }^{5}$}<smiles>CC(=O)CCc1c[nH]c2ccc(Br)cc12</smiles>

(white solid, Hexane/EtOAc $=4: 1$, yield 92\%); ${ }^{1} \mathrm{H}$ NMR $\delta 2.17(s, 3 \mathrm{H}), 2.84(t, 2 \mathrm{H}, J=7.3 \mathrm{~Hz}$ ), $3.01(t, 2 \mathrm{H}, J=7.3 \mathrm{~Hz}), 7.00(d, 1 \mathrm{H}, J=2.3 \mathrm{~Hz}), 7.22-7.30(\mathrm{~m}, 2 \mathrm{H}), 7.72(d, 1 \mathrm{H}, J=1.4 \mathrm{~Hz}), 8.11$ $(b r, 1 \mathrm{H}) ;{ }^{13} \mathrm{C}$ NMR $\delta 19.0,30.1,43.9,112.5,112.6,114.9,121.3,122.8,124.8,128.9,134.9$, 208.5.

4-(5-Methyl-3-indolyl)-2-butanone (3):<smiles>CC(=O)CCc1c[nH]c2ccc(C)cc12</smiles>

(White solid, Hexane/EtOAc = 3:1, 94\%); melting point: $94-95{ }^{\circ} \mathrm{C} .{ }^{1} \mathrm{H}$ NMR $\delta 2.17(s, 3 \mathrm{H}), 2.49$ (s, 3H), $2.86(\mathrm{t}, \mathrm{J}=7.8 \mathrm{~Hz}, 2 \mathrm{H}), 3.05(\mathrm{t}, \mathrm{J}=7.4 \mathrm{~Hz}, 2 \mathrm{H}), 6.95(\mathrm{~d}, \mathrm{~J}=0.9 \mathrm{~Hz}, 1 \mathrm{H}), 7.05(\mathrm{~d}, \mathrm{~J}=8.2$ $\mathrm{Hz}, 1 \mathrm{H}), 7.25(\mathrm{~d}, \mathrm{~J}=8.2 \mathrm{~Hz}, 1 \mathrm{H}), 7.39(\mathrm{~s}, 1 \mathrm{H}), 7.93(b r, 1 \mathrm{H}) ;{ }^{13} \mathrm{C}$ NMR $\delta$ 19.4, 21.5, 30.0, 44.1, 110.8, 114.6, 118.2, 121.6, 123.6, 127.3, 128.4, 134.6, 208.9. IR (KBr) 3326, 1705, 1560, 1442, 1317, 1159, 804, $788 \mathrm{~cm}^{-1}$. Anal. Calcd for $\mathrm{C}_{13} \mathrm{H}_{15} \mathrm{NO}$ : C, 77.58; H, 7.51; N, 6.96. Found: C, $77.55 ; \mathrm{H}, 7.64, \mathrm{~N}, 6.77$.

4-(4-Methyl-3-indolyl)-2-butanone (4): ${ }^{6}$<smiles>COc1cccc2[nH]cc(CCC(C)=O)c12</smiles>

(White solid, Hexane/EtOAc $=3: 1,83 \%$ ); melting point: $116-117{ }^{\circ} \mathrm{C} ;{ }^{1} \mathrm{H}$ NMR $\delta 2.12(\mathrm{~s}, 3 \mathrm{H})$, $2.84(\mathrm{t}, \mathrm{J}=7.3 \mathrm{~Hz}, 2 \mathrm{H}), 3.14(\mathrm{t}, \mathrm{J}=7.6 \mathrm{~Hz}, 2 \mathrm{H}), 3.91(\mathrm{~s}, 3 \mathrm{H}), 6.48(\mathrm{~d}, \mathrm{~J}=7.4 \mathrm{~Hz}, 1 \mathrm{H}), 6.81(\mathrm{~s}$, 1h), $6.93(\mathrm{~d}, \mathrm{~J}=8.2 \mathrm{~Hz}, 1 \mathrm{H}), 7.07$ (t, J = 8.0 Hz, 1H), $8.02(\mathrm{br}, 1 \mathrm{H}) ;{ }^{13} \mathrm{C} \mathrm{NMR} \delta 21.4,29.9,45.7$, $55.1,99.3,104.5,115.5,117.1,120.4,122.7,138.1,154.6,209.7$.

4-(1-1H-3-Indolyl)-2-butanone (5): ${ }^{4}$<smiles>CC(=O)CCc1c[nH]c2ccccc12</smiles> 
(white solid, Hexane/EtOAc $=3: 1,96 \%) ;{ }^{1} \mathrm{H}$ NMR $\delta 2.18(s, 3 \mathrm{H}), 2.88(t, 2 \mathrm{H}, J=7.3 \mathrm{~Hz}), 3.10(t$, $2 \mathrm{H}, \mathrm{J}=7.3 \mathrm{~Hz}), 6.99(s, 1 \mathrm{H}), 7.15-7.28(m, 2 \mathrm{H}), 7.37(d, 1 \mathrm{H}, J=8.2 \mathrm{~Hz}), 7.63(d, 1 \mathrm{H}, J=7.8 \mathrm{~Hz})$, 8.09 (br, 1H); ${ }^{13} \mathrm{C}$ NMR $\delta 19.28,29.99,44.00,111.13,114.97,118.56,119.18,121.46,121.93$, 127.08, 136.23, 208.91.

4-(5-Methoxy-3-indolyl)-2-butanone (6): ${ }^{5}$<smiles>COc1ccc2[nH]cc(CCC(C)=O)c2c1</smiles>

(White solid, Hexane/EtOAc $=3: 1$, yield 90\%); ${ }^{1} \mathrm{H}$ NMR $\delta 2.17(s, 3 \mathrm{H}), 2.86(t, 2 \mathrm{H}, J=7.3 \mathrm{~Hz})$, $3.03(t, 2 \mathrm{H}, J=7.3 \mathrm{~Hz}), 3.89(s, 3 \mathrm{H}), 6.87(d, 1 \mathrm{H}, J=8.7 \mathrm{~Hz}), 6.97(s, 1 \mathrm{H}), 7.04(s, 1 \mathrm{H}), 7.25(d$, $1 \mathrm{H}, J=8.7 \mathrm{~Hz}), 7.98(b r, 1 \mathrm{H}) ;{ }^{13} \mathrm{C}$ NMR $\delta 19.27,30.03,43.88,55.92,100.50,111.85,112.13$, $114.80,122.20,127.50,131.39,153.87,208.85$.

4-(5-Chloro-3-indolyl)-2-butanone (7):<smiles>CC(=O)CCc1c[nH]c2ccc(Cl)cc12</smiles>

(White solid, Hexane/EtOAc $=3: 1,97 \%$ ); melting point: 82-83 ${ }^{\circ} \mathrm{C} ;{ }^{1} \mathrm{H}$ NMR $\delta 2.15$ (s, 3H), 2.83 (t, J = 7.3 Hz, 2H), $3.00(\mathrm{t}, \mathrm{J}=7.3 \mathrm{~Hz}, 2 \mathrm{H}), 6.99(\mathrm{~s}, 1 \mathrm{H}), 7.14\left(\mathrm{dd}, \mathrm{J}_{\mathrm{a}}=8.7 \mathrm{~Hz}, \mathrm{~J}_{\mathrm{b}}=1.8 \mathrm{~Hz}, 1 \mathrm{H}\right)$, $7.25(\mathrm{~d}, \mathrm{~J}=8.2 \mathrm{~Hz}, 1 \mathrm{H}), 7.55(\mathrm{~d}, \mathrm{~J}=1.8 \mathrm{~Hz}, 1 \mathrm{H}), 8.18(\mathrm{br}, 1 \mathrm{H}) ;{ }^{13} \mathrm{C}$ NMR $\delta 19.1,30.0,43.8$, 112.2, 114.8, 118.1, 122.2, 123.0, 124.9, 128.2, 134.6, 208.6; IR (KBr) 3352, 1700, 1457, 1358, 1166, $1058 \mathrm{~cm}^{-1}$. Anal. Calcd for $\mathrm{C}_{12} \mathrm{H}_{12} \mathrm{ClNO}$ C, 65.02; H, 5.46; N, 6.32. Found: C, 64.73; H, 5.37, N, 6.24 .

3-(1-1H-3-Indolyl)-cyclopentanone (8): ${ }^{7}$<smiles>O=C1CCC(c2c[nH]c3ccccc23)C1</smiles>

(oil, Hexane/EtOAc $=3: 1,70 \%) ;{ }^{1} \mathrm{H}$ NMR $\delta$ 2.10-2.20 $(m, 1 \mathrm{H}), 2.29-2.56(m, 4 \mathrm{H}), 2.75-2.81(m$, 1H), $3.74(p, 1 \mathrm{H}, J=7.8 \mathrm{~Hz}), 6.99(s, 1 \mathrm{H}), 7.16(t, 1 \mathrm{H}, J=7.1 \mathrm{~Hz}), 7.24(t, 1 \mathrm{H}, J=7.6 \mathrm{~Hz}), 7.39$ $(d, 1 \mathrm{H}, J=8.3 \mathrm{~Hz}), 7.65(d, 1 \mathrm{H}, J=7.8 \mathrm{~Hz}), 8.07(b r, 1 \mathrm{H}) ;{ }^{13} \mathrm{C} \mathrm{NMR} \delta 29.87,33.71,38.15,45.28$, $111.34,118.59,119.07,119.46,119.91,122.33,126.60,136.68,219.40$. 
1-(1H-Indol-3-yl)-pentan-3-one (9): ${ }^{8}$<smiles>CCC(=O)CCc1c[nH]c2ccccc12</smiles>

(White solid, Hexane/EtOAc $=3: 1,89 \%) ;{ }^{1} \mathrm{H} \operatorname{NMR} \delta 0.96(t, 3 \mathrm{H}, J=7.3 \mathrm{~Hz}), 2.33\left(d d, 2 \mathrm{H}, J_{a}=\right.$ $\left.7.4 \mathrm{~Hz}, J_{b}=7.3 \mathrm{~Hz}\right), 2.74(t, 2 \mathrm{H}, J=7.3 \mathrm{~Hz}), 2.98(t, 2 \mathrm{H}, J=7.3 \mathrm{~Hz}), 6.88(d, 1 \mathrm{H}, J=2.3 \mathrm{~Hz})$ 7.02-7.16 $(m, 2 \mathrm{H}), 7.26(d, 1 \mathrm{H}, J=7.8 \mathrm{~Hz}), 7.52(d, 1 \mathrm{H}, J=7.8 \mathrm{~Hz}), 7.93(b r, 1 \mathrm{H}) ;{ }^{13} \mathrm{C}$ NMR $\delta$ $7.74,19.40,36.05,42.75,111.12,115.25,118.64,119.22,121.46,121.96,127.14,136.27,211.49$. 3-(1 H-3-Indolyl)-butyrophenone (10): ${ }^{9}$<smiles>CC(CC(=O)c1ccccc1)c1c[nH]c2ccccc12</smiles>

(oil, Hexane/EtOAc = 3:1, 88\%); ${ }^{1} \mathrm{H}$ NMR $\delta 1.51(d, J=6.9 \mathrm{~Hz}, 3 \mathrm{H}), 3.30(d d, J=7.3 \mathrm{~Hz}, J=$ $9.2 \mathrm{~Hz}, 1 \mathrm{H}), 3.53(d d, J=5.0 \mathrm{~Hz}, J=11.5 \mathrm{~Hz}, 1 \mathrm{H}), 3.84-3.93(m, 1 \mathrm{H}), 7.03(d, J=2.3 \mathrm{~Hz}, 1 \mathrm{H})$, 7.14-7.29 (m, 2H), $7.39(d, J=8.2 \mathrm{~Hz}, 1 \mathrm{H}), 7.44-7.50(m, 2 \mathrm{H}), 7.54-7.60(m, 1 \mathrm{H}), 7.73(d, J=7.8$ $\mathrm{Hz}, 1 \mathrm{H}), 7.99-8.01(m, 2 \mathrm{H}) ; 8.08(b r, 1 \mathrm{H}) ;{ }^{13} \mathrm{C}$ NMR $\delta 20.97,27.12,46.42,111.26,119.16$, $119.18,120.18,121.42,121.95,126.28,128.06,128.50,132.88,136.51,137.26,199.78$.

3-(1 H-3-Indolyl)-valerophenone (11): ${ }^{9}$<smiles>CCC(CC(=O)c1ccccc1)c1c[nH]c2ccccc12</smiles>

$($ Colorless oil, Hexane/EtOAc $=3: 1,43 \%) ;{ }^{1} \mathrm{H}$ NMR $\delta 0.80(\mathrm{t}, \mathrm{J}=7.3 \mathrm{~Hz}, 3 \mathrm{H}), 1.78(\mathrm{~m}, 2 \mathrm{H}), 3.31$ (m, 2H), $3.52(\mathrm{~m}, 1 \mathrm{H}), 6.92(\mathrm{~d}, \mathrm{~J}=2.3 \mathrm{~Hz}, 1 \mathrm{H}), 7.00-7.17(\mathrm{~m}, 2 \mathrm{H}), 7.25(\mathrm{~d}, \mathrm{~J}=8.2 \mathrm{~Hz}, 1 \mathrm{H})$, 7.30-7.35 (m, 2H), 7.41-7.45 (m, 1H), 7.61 (d, J = 7.8 Hz, 1H), 7.83 (d, J = 6.9 Hz, 2H), 7.89 (br, $1 \mathrm{H}) ;{ }^{13} \mathrm{C}$ NMR $\delta 12.2,28.3,34.5,44.8,111.2,119.1,119.4,121.3,121.8,126.7,128.1,128.4$, $132.8,136.6,137.3,200.0$.

Methyl 3-(3-oxobutyl)indole-5-carboxylate (12) 
(1)

(White solid, Hexane/EtOAc $=1: 1,80 \%$ ); melting point: $106-108{ }^{\circ} \mathrm{C} ;{ }^{1} \mathrm{H}$ NMR $\delta 2.12(\mathrm{~s}, 3 \mathrm{H})$, $2.82(\mathrm{t}, \mathrm{J}=7.3 \mathrm{~Hz}, 2 \mathrm{H}), 3.05(\mathrm{t}, \mathrm{J}=7.6 \mathrm{~Hz}, 2 \mathrm{H}), 3.92(\mathrm{~s}, 3 \mathrm{H}), 7.02(\mathrm{~s}, 1 \mathrm{H}), 7.32(\mathrm{~d}, \mathrm{~J}=8.7 \mathrm{~Hz}$, 1H), $7.87\left(\mathrm{dd}, \mathrm{J}_{\mathrm{a}}=8.2 \mathrm{~Hz}, \mathrm{~J}_{\mathrm{b}}=1.4 \mathrm{~Hz}, 1 \mathrm{H}\right), 8.33(\mathrm{~s}, 1 \mathrm{H}), 8.37(\mathrm{br}, 1 \mathrm{H}) ;{ }^{13} \mathrm{C}$ NMR $\delta$ 19.1, 30.0, 43.9, 51.9, 110.8, 116.6, 121.3, 121.7, 122.9, 123.4, 126.8, 138.9, 168.2, 208.5; IR (KBr) 3317, $1712,1618,1350,1282,1217,1110 \mathrm{~cm}^{-1}$. Anal. Calcd for $\mathrm{C}_{14} \mathrm{H}_{15} \mathrm{NO}_{3}$ : C, 68.56; H, 6.16; N, 5.71. Found: C, 68.35; H, 6.08, N, 5.60.

3-(1-Phenyl-2-nitroethyl)-1-methyl-1H-indole (13): ${ }^{10}$<smiles>Cn1cc(C(C[N+](=O)[O-])c2ccccc2)c2ccccc21</smiles>

(oil, Hexane/EtOAc $=3: 1,68 \%) ;{ }^{1} \mathrm{H}$ NMR $\delta 3.62(\mathrm{~s}, 3 \mathrm{H}), 4.80-4.86(\mathrm{~m}, 1 \mathrm{H}), 4.92-4.97(\mathrm{~m}, 1 \mathrm{H})$, $5.08(\mathrm{t}, \mathrm{J}=8.3 \mathrm{~Hz}, 1 \mathrm{H}), 6.76,(\mathrm{~s}, 1 \mathrm{H}), 6.96-7.00(\mathrm{~m}, 1 \mathrm{H}), 7.11-7.26(\mathrm{~m}, 7 \mathrm{H}), 7.36(\mathrm{~d}, \mathrm{~J}=8.2 \mathrm{~Hz}$, $1 \mathrm{H}) ;{ }^{13} \mathrm{C}$ NMR $\delta 32.8,41.5,79.5,109.5,112.7,118.9,119.4,122.2,126.3,126.5,127.4,127.7$, $128.8,137.2,139.3$

3-[1-(4-Methoyphenyl)-2-nitroethyl]-1-methyl-1H-indole (14): ${ }^{10}$<smiles>COc1ccc(C(C[N+](=O)[O-])c2cn(C)c3ccccc23)cc1</smiles>

(oil, Hexane/EtOAc = 2:1, 75\%); ${ }^{1} \mathrm{H}$ NMR $\delta 3.73(\mathrm{~s}, 3 \mathrm{H}), 3.76(\mathrm{~s}, 3 \mathrm{H}), 4.88\left(\mathrm{dd}, \mathrm{J}_{\mathrm{a}}=12.4 \mathrm{~Hz}, \mathrm{~J}_{\mathrm{b}}=\right.$ $8.7 \mathrm{~Hz}, 1 \mathrm{H}), 5.02\left(\mathrm{dd}, \mathrm{J}_{\mathrm{a}}=12.4 \mathrm{~Hz}, \mathrm{~J}_{\mathrm{b}}=7.8 \mathrm{~Hz}, 1 \mathrm{H}\right), 5.13(\mathrm{t}, \mathrm{J}=7.8 \mathrm{~Hz}, 1 \mathrm{H}), 6.85,(\mathrm{~d}, \mathrm{~J}=8.7 \mathrm{~Hz}$, 3H), $7.07(\mathrm{t}, \mathrm{J}=7.4 \mathrm{~Hz}, 1 \mathrm{H}), 7.20-7.30(\mathrm{~m}, 4 \mathrm{H}), 7.44(\mathrm{~d}, \mathrm{~J}=7.8 \mathrm{~Hz}, 1 \mathrm{H}) ;{ }^{13} \mathrm{C}$ NMR $\delta 32.8,40.8$, $55.2,79.7,109.4,113.1,114.2,119.0,119.4,122.1,126.2,126.5,128.7,131.3,137.3,158.8$.

3-(1-Furan-2-yl-2-nitroethyl)-1-methyl-1 $H$-indole (15): ${ }^{10}$ 
<smiles>Cn1cc(C(C[N+](=O)[O-])c2ccco2)c2ccccc21</smiles>

(oil, Hexane/EtOAc = 2:1, 81\%); ${ }^{1} \mathrm{H}$ NMR $\delta 3.73(\mathrm{~s}, 3 \mathrm{H}), 4.89\left(\mathrm{dd}, \mathrm{J}_{\mathrm{a}}=12.4 \mathrm{~Hz}, \mathrm{~J}_{\mathrm{b}}=7.4 \mathrm{~Hz}, 1 \mathrm{H}\right)$, $5.03\left(\mathrm{dd}, \mathrm{J}_{\mathrm{a}}=12.4 \mathrm{~Hz}, \mathrm{~J}_{\mathrm{b}}=8.3 \mathrm{~Hz}, 1 \mathrm{H}\right), 5.23(\mathrm{t}, \mathrm{J}=7.8 \mathrm{~Hz}, 1 \mathrm{H}), 6.16,(\mathrm{~d}, \mathrm{~J}=3.2 \mathrm{~Hz}, 1 \mathrm{H}), 6.30$ $\left(\mathrm{dd}, \mathrm{J}_{\mathrm{a}}=3.2 \mathrm{~Hz}, \mathrm{~J}_{\mathrm{b}}=1.8 \mathrm{~Hz}, 1 \mathrm{H}\right), 6.97(\mathrm{~s}, 1 \mathrm{H}), 7.12\left(\mathrm{td}, \mathrm{J}_{\mathrm{a}}=6.9 \mathrm{~Hz}, \mathrm{~J}_{\mathrm{b}}=0.9 \mathrm{~Hz}, 1 \mathrm{H}\right), 7.22-7.31$ $(\mathrm{m}, 2 \mathrm{H}), 7.37(\mathrm{~d}, \mathrm{~J}=1.4 \mathrm{~Hz}, 1 \mathrm{H}), 7.54(\mathrm{~d}, \mathrm{~J}=8.2 \mathrm{~Hz}, 1 \mathrm{H}) ;{ }^{13} \mathrm{C}$ NMR $\delta 32.8,35.6,77.9,107.2$, 109.6, 109.9, 110.4, 118.7, 119.5, 122.1, 126.1, 127.3, 137.1, 142.2, 152.3.

\section{1-Methyl-3-(2-nitro-1-thiophen-2-ylethyl)-1 $H$-indole (16): ${ }^{10}$}<smiles>Cn1cc(C(C[N+](=O)[O-])c2cccs2)c2ccccc21</smiles>

(oil, Hexane/EtOAc = 2:1, 80\%); ${ }^{1} \mathrm{H}$ NMR $\delta 3.74(\mathrm{~s}, 3 \mathrm{H}), 4.99\left(\mathrm{ddd}, \mathrm{J}_{\mathrm{a}}=20.1 \mathrm{~Hz}, \mathrm{~J}_{\mathrm{b}}=12.8 \mathrm{~Hz}, \mathrm{~J}_{\mathrm{c}}\right.$ $=7.8 \mathrm{~Hz}, 2 \mathrm{H}), 5.44(\mathrm{t}, \mathrm{J}=7.8 \mathrm{~Hz}, 1 \mathrm{H}), 6.92-6.98,(\mathrm{~m}, 3 \mathrm{H}), 7.10\left(\mathrm{td}, \mathrm{J}_{\mathrm{a}}=6.9 \mathrm{~Hz}, \mathrm{~J}_{\mathrm{b}}=0.9 \mathrm{~Hz}, 1 \mathrm{H}\right)$, 7.18-7.31 (m, 3H), $7.50(\mathrm{~d}, \mathrm{~J}=7.8 \mathrm{~Hz}, 1 \mathrm{H}) ;{ }^{13} \mathrm{C}$ NMR $\delta 32.9,36.9,80.1,109.6,112.4,118.9$, $119.6,122.3,124.8,125.1,126.2,126.6,126.9,137.2,143.1$.

3-(2-Methyindol-3-yl)cyclohexanone (17): ${ }^{11}$<smiles>Cc1[nH]c2ccccc2c1C1CCCC(=O)C1</smiles>

(oil, Hexane/EtOAc $=3: 1,70 \%) ;{ }^{1} \mathrm{H}$ NMR $\delta$ 1.71-1.82 $(m, 1 \mathrm{H}), 1.96-2.02(m, 1 \mathrm{H}), 2.15-2.33(m$, 2H), $2.34(\mathrm{~s}, 3 \mathrm{H}), 2.35-2.50(\mathrm{~m}, 3 \mathrm{H}), 2.93-2.99(\mathrm{~m}, 1 \mathrm{H}), 3.14-3.21(\mathrm{~m}, 1 \mathrm{H})$, 7.01-7.10 (m, $2 \mathrm{H})$, 7.22-7.26 (m, 1H), 7.62 (d, J = 7.8 Hz, 1H), $7.82($ brs, $1 \mathrm{H}) ;{ }^{13} \mathrm{C}$ NMR $\delta 12.0,26.1,31.5,37.3,41.4$, $48.1,110.6,113.8,118.8,119.1,120.9,126.8,130.1,135.4,211.8$.

\section{Tert-butyl 3-(3-oxobutyl)indole-5-carbamate (18)}


<smiles>CC(=O)CCc1c[nH]c2ccc(NC(=O)OC(C)(C)C)cc12</smiles>

(White solid, Hexane/EtOAc $=1: 1,94 \%$ ); melting point: 97-99 ${ }^{\circ} \mathrm{C} .{ }^{1} \mathrm{H}$ NMR $\delta 1.58(\mathrm{~s}, 9 \mathrm{H}), 2.14$ (s, 3H), $2.82(\mathrm{t}, \mathrm{J}=7.3 \mathrm{~Hz}, 2 \mathrm{H}), 3.01(\mathrm{t}, \mathrm{J}=7.4 \mathrm{~Hz}, 2 \mathrm{H}), 6.61(\mathrm{~s}, 1 \mathrm{H}), 6.93(\mathrm{~s}, 1 \mathrm{H}), 7.10(\mathrm{~d}, \mathrm{~J}=8.3$ $\mathrm{Hz}, 1 \mathrm{H}), 7.23(\mathrm{~d}, \mathrm{~J}=8.7 \mathrm{~Hz}, 1 \mathrm{H}), 7.66(\mathrm{~s}, 1 \mathrm{H}), 8.22$ (brs, 1H); ${ }^{13} \mathrm{C}$ NMR $\delta$ 19.2, 28.4, 29.9, 43.9, 79.9, 109.4, 111.3, 114.9, 115.7, 122.5, 127.3, 130.3, 133.2, 153.6, 209.0. IR (KBr) 3399, 3321, 2980, 1708, 1541, 1241, 1167, $668 \mathrm{~cm}^{-1}$; HRMS Calculated for $\mathrm{C}_{17} \mathrm{H}_{22} \mathrm{~N}_{2} \mathrm{O}_{3}\left[\mathrm{M}+\mathrm{Na}{ }^{+}\right]: 325.1523$ Found: 325.1518 .

4-(5-(4,4,5,5-Tetramethyl-1,3,2-dioxaborolan-2-yl)-3-indolyl)-2-butanone (19)<smiles>CC(=O)CCc1c[nH]c2ccc(B3OC(C)(C)C(C)(C)O3)cc12</smiles>

(White solid, Hexane/EtOAc $=3: 1,85 \%$ ); melting point: $136-137{ }^{\circ} \mathrm{C} .{ }^{1} \mathrm{H}$ NMR $\delta 1.32(\mathrm{~s}, 12 \mathrm{H})$, $2.06(\mathrm{~s}, 3 \mathrm{H}), 2.76(\mathrm{t}, \mathrm{J}=7.3 \mathrm{~Hz}, 2 \mathrm{H}), 2.99$ (t, J = $7.3 \mathrm{~Hz}, 2 \mathrm{H}), 6.87(\mathrm{~s}, 1 \mathrm{H}), 7.26(\mathrm{~d}, \mathrm{~J}=8.2 \mathrm{~Hz}$, 1H), 7.59 (d, J = 8.2 Hz, 1H), 8.06 (s, 1H), 8.20 (brs, $1 \mathrm{H}) ;{ }^{13} \mathrm{C}$ NMR $\delta$ 19.2, 24.8, 29.9, 44.1, 83.4, 110.6, 115.6, 121.6, 126.3, 126.9, 128.2, 138.4, 208.9. IR (KBr) 3337, 3271, 2982, 1696, 1514, $1438,1374,1353,1137,1091,962,689 \mathrm{~cm}^{-1}$; HRMS Calculated for $\mathrm{C}_{18} \mathrm{H}_{24} \mathrm{BNO}_{3}\left[\mathrm{M}+\mathrm{H}^{+}\right]$: 314.1922 Found: 314.1931.

4-[1-(2-Hydroxyethyl)-3-indolyl]-2-butanone (20):<smiles>CC(=O)CCc1cn(CCO)c2ccccc12</smiles>

(Oil, Hexane/EtOAc = 1:2, 85\%); ${ }^{1} \mathrm{H}$ NMR $\delta 2.06$ (brs, 1H), 2.16 (s, 3H), 2.85 (t, J = $7.8 \mathrm{~Hz}, 2 \mathrm{H}$ ), $3.05(\mathrm{t}, \mathrm{J}=7.8 \mathrm{~Hz}, 2 \mathrm{H}), 3.90(\mathrm{t}, \mathrm{J}=5.0 \mathrm{~Hz}, 2 \mathrm{H}), 4.20(\mathrm{t}, \mathrm{J}=5.5 \mathrm{~Hz}, 2 \mathrm{H}), 6.96(\mathrm{~s}, 1 \mathrm{H}), 7.14(\mathrm{t}, \mathrm{J}=$ $8.2 \mathrm{~Hz}, 1 \mathrm{H}), 7.24(\mathrm{t}, \mathrm{J}=6.9 \mathrm{~Hz}, 1 \mathrm{H}), 7.35(\mathrm{~d}, \mathrm{~J}=8.2 \mathrm{~Hz}, 1 \mathrm{H}), 7.61(\mathrm{~d}, \mathrm{~J}=7.8 \mathrm{~Hz}, 1 \mathrm{H}) ;{ }^{13} \mathrm{C} \mathrm{NMR}$ $\delta 19.1,29.9,44.0,48.4,61.8,109.3,114.0,118.8,118.9,121.7,125.6,127.8,136.4,208.9$. IR (KBr) $3447,2928,1712,1469,1361,1168,1057,774 \mathrm{~cm}^{-1}$; HRMS Calculated for $\mathrm{C}_{14} \mathrm{H}_{17} \mathrm{NO}_{2}$ 
$\left[\mathrm{M}+\mathrm{Na}^{+}\right]: 232.1332$ Found: 232.1339.

4-(5-Benzyloxy-3-indolyl)-2-butanone (21)<smiles>CC(=O)CCc1c[nH]c2ccc(OCc3ccccc3)cc12</smiles>

(White solid, Hexane/EtOAc $=2: 1,91 \%$ ); melting point: $119-120{ }^{\circ} \mathrm{C},{ }^{1} \mathrm{H}$ NMR $\delta 2.12(\mathrm{~s}, 3 \mathrm{H})$, $2.79(\mathrm{t}, \mathrm{J}=7.8 \mathrm{~Hz}, 2 \mathrm{H}), 2.98(\mathrm{t}, \mathrm{J}=7.8 \mathrm{~Hz}, 2 \mathrm{H}), 5.10(\mathrm{~s}, 2 \mathrm{H}), 6.91-6.94(\mathrm{~m}, 2 \mathrm{H}), 7.09(\mathrm{~s}, 1 \mathrm{H})$, 7.22-7.24 (m, 2H), 7.27-7.32 (m, 1H), 7.35-7.39 (m, 2H), 7.47 (d, J = 7.8 Hz, 2H), 7.81 (brs, 1H); ${ }^{13} \mathrm{C}$ NMR $\delta 19.3,30.0,43.9,71.0,102.3,111.8,112.8,114.8,122.3,127.5,127.6,127.7,128.5$, 131.6, 137.6, 153.0, 208.9. IR (KBr) 3386, 2916, 1709, 1482, 1186, 1005, $714 \mathrm{~cm}^{-1}$; Anal. Calcd for $\mathrm{C}_{19} \mathrm{H}_{19} \mathrm{NO}_{2}$ : C, 77.79; H, 6.53; N, 4.77. Found: C, 77.99; H, 6.73, N, 4.70.

4-(5-Dibenzylamino-1 H-indol-3-yl)-butan-2-one (22): ${ }^{6}$<smiles>CC(=O)CCc1c[nH]c2ccc(N(Cc3ccccc3)Cc3ccccc3)cc12</smiles>

(White solid, Hexane/EtOAc = 2:1, 74\%); ${ }^{1} \mathrm{H}$ NMR $\delta 2.03(\mathrm{~s}, 3 \mathrm{H}), 2.63(\mathrm{t}, \mathrm{J}=7.8 \mathrm{~Hz}, 2 \mathrm{H}), 2.87$ (t, $\mathrm{J}=6.8 \mathrm{~Hz}), 4.60(\mathrm{~s}, 4 \mathrm{H}), 7.81-7.86(\mathrm{~m}, 3 \mathrm{H}), 7.14-7.30(\mathrm{~m}, 11 \mathrm{H}), 7.68(\mathrm{brs}, 1 \mathrm{H}) ;{ }^{13} \mathrm{C}$ NMR $\delta 19.4$, $29.9,43.7,55.9,102.9,111.6,112.3,114.3,121.9,126.7,127.2,127.8,128.4,130.4,139.4,143.6$, 208.9.

3-(3-Oxocyclopentyl)-1 H-indole-5-carbonitrile (23): ${ }^{12}$<smiles>N#Cc1ccc2[nH]cc(C3CCC(=O)C3)c2c1</smiles>

(Oil, Hexane/EtOAc $=2: 1,85 \%) ;{ }^{1} \mathrm{H}$ NMR $\delta$ 2.03-2.12 $(\mathrm{m}, 1 \mathrm{H}), 2.28-2.46(\mathrm{~m}, 3 \mathrm{H}), 2.51-2.57(\mathrm{~m}$, $1 \mathrm{H}), 2.75\left(\mathrm{dd}, \mathrm{J}_{a}=18.3, \mathrm{~J}_{b}=7.8 \mathrm{~Hz}, 1 \mathrm{H}\right), 3.68(\mathrm{~m}, 1 \mathrm{H}), 7.11(\mathrm{~d}, \mathrm{~J}=2.3 \mathrm{~Hz}, 1 \mathrm{H}), 7.39-7.45(\mathrm{~m}$, 2H), 7.95 (s, 1H), 8.87 (brs, 1H); ${ }^{13} \mathrm{C}$ NMR $\delta 29.9,33.3,38.0,45.0,102.2,112.3,119.3,120.7$, $122.3,124.7,125.1,126.5,138.4,218.7$. 


\section{References}

1. Jones, C. W.; Tsuji, K; Davis, M. E. Nature 1998, 393, 52;

2. Li, P.-H.; Wang, L. Adv. Synth. Catal. 2006, 348, 681.

3. Sow, B.; Hamoudi, S.; Zahedi-Niaki, H.; Kaliaguine, S. Miropor. Mesopor. Mater. 2005, 79, 129.

4. Komoto, I.; Kobayashi. S. Org. Lett. 2002, 4 (7), 1115.

5. Bartoli, G.; Bartolacci, M.; Bosco, M.; Foglia, G.; Giuliani, A.; Marcantoni, E.; Sambri, L. and Torregiani, E. J. Org. Chem. 2003, 68 (11),6594.

6. Trost, B. M.; Quancard, J. J. Am. Chem. Soc. 2006, 128, 6314.

7. Rajabi, F.; Saidi. M. R. J. Sulfur Chem. 2005, 26, 251.

8. Ferreira, E. M. and Stoltz, B. M. J. Am. Chem. Soc. 2003, 125, 9578.

9. Wabnitz, T. C.; Yu, J. Q.; Spencer, J. B. Chem. Eur. J. 2004, 10, 484.

10. Wei, Z.; Rita, G. H.; Jorgensen, K. A. Org. Biomol. Chem. 2005, 3, 2566.

11. Kantam, M. L.; Laha, S.; Yadav, J.; Choudary, B. M.; Screedhar, B. Adv. Syn. Catal. 2006, $348,867$.

12. King, D.; Deskus, J. A.; Macor, J. E.; Mattson, R. J.; Meng, Z. Sloan, C. P. WO 2004026236, 2004. 


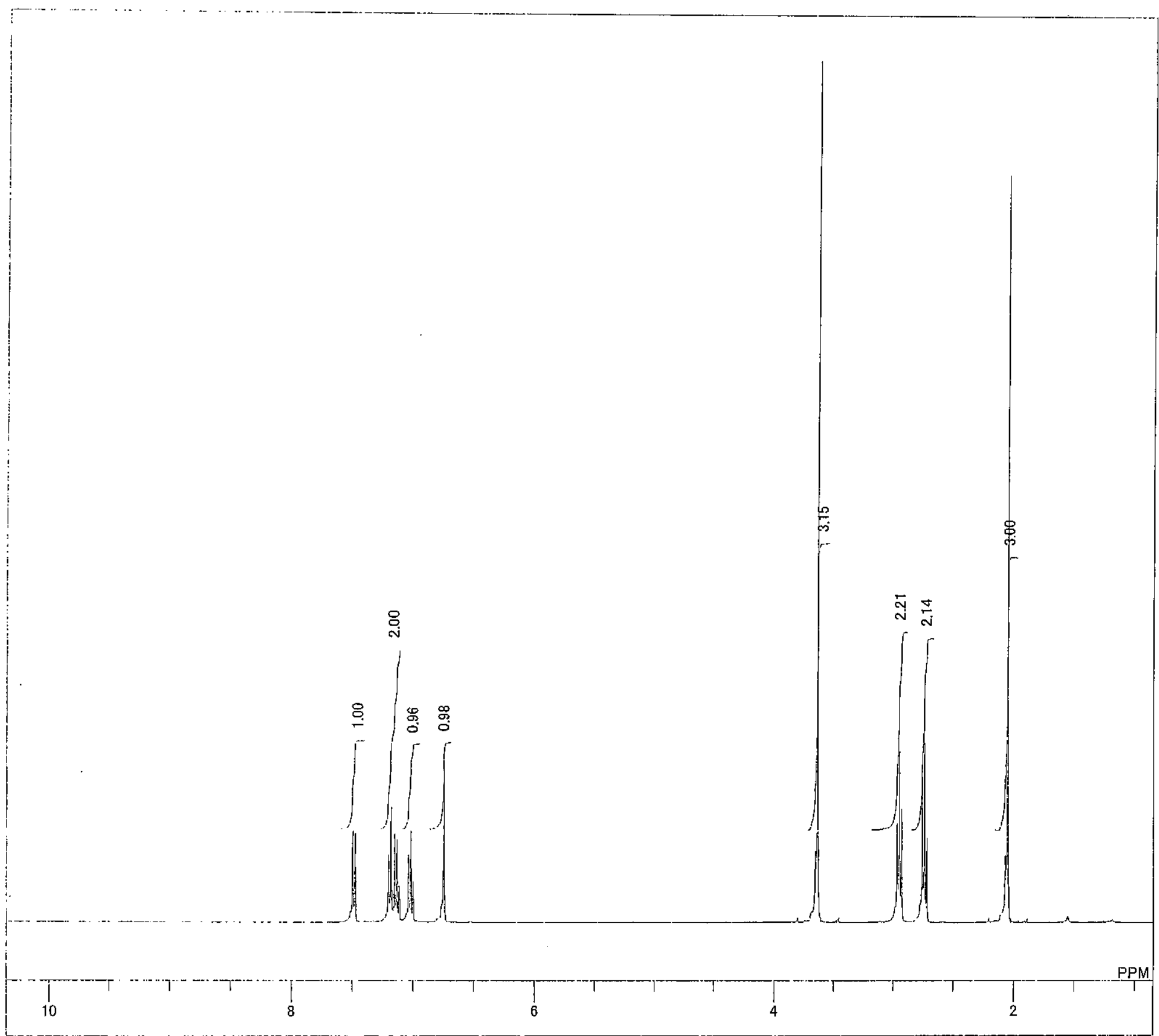

OBNUC

EXMOD

OBFRQ

OBFIN

POINT

FREQU

SCANS

ACQTM

PD
PW1

IRNUC

CTEMP

STEMP

EXREF

RGAIN

*Eca $¥$ data $¥ G u ¥ 333$.

02-03-2006 15:20:41

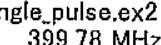
$399.78 \mathrm{MHz}$
$4.19 \mathrm{KHz}$ $7.29 \mathrm{~Hz}$ 20480 $9378.89 \mathrm{~Hz}$

$2.1837 \mathrm{sec}$

$2.0000 \mathrm{sec}$
$5.50 \mathrm{usec}$

tH

$\operatorname{CDCL} 3.3$

$0.00 \mathrm{ppm}$

$0.12 \mathrm{~Hz}$

32

$\frac{1}{n}$

4-(1-Methyl-3-indolyl)-2-butanone (1)

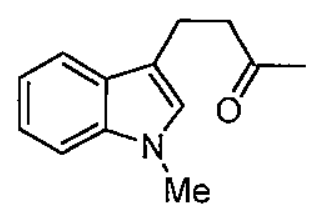


$¥ ¥$ Eca $¥$ data $¥ G u ¥ 333-13 \mathrm{C} .1$

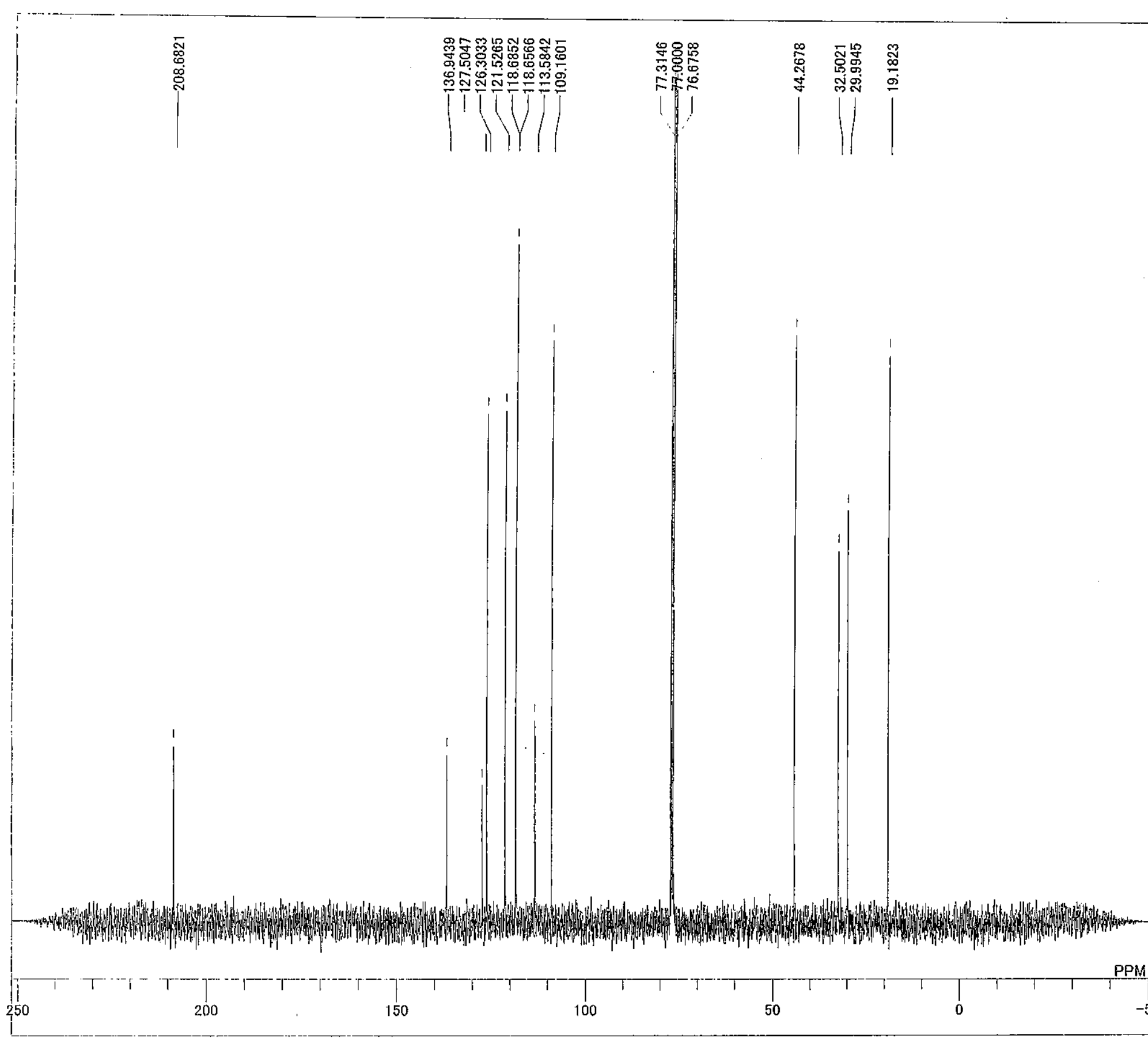

DFILE

DATIM

OBNUO

EXMOD

OBFRQ

OBSET

PBINT

PONT

FREQU

ACQTM

PD

PW1

IRNUC

SLVNT

RGAIN
¥¥Eca $¥$ data $¥ \mathrm{Gu} ¥ 333-13 \mathrm{C} .1$

02-03-2006 15:27:46

ingle_pulse_dec $00.53 \mathrm{MHz}$ $5.35 \mathrm{KHz}$ $500 \mathrm{~Hz}$ $39259.39 \mathrm{~Hz}$

39259.39
$8 \mathrm{~Hz}$ $1.0433 \mathrm{sec}$ $2.0000 \mathrm{sec}$

$1 \mathrm{H}$ 3.33 usec

$\mathrm{CDCL}^{24.6}$

$77.00 \mathrm{ppm}$ $0.12 \mathrm{~Hz}$ $\frac{m}{\dot{m}}$

4-(1-Methyl-3-indolyl)-2-butanone (1)

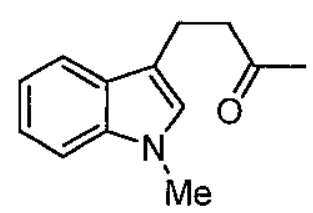




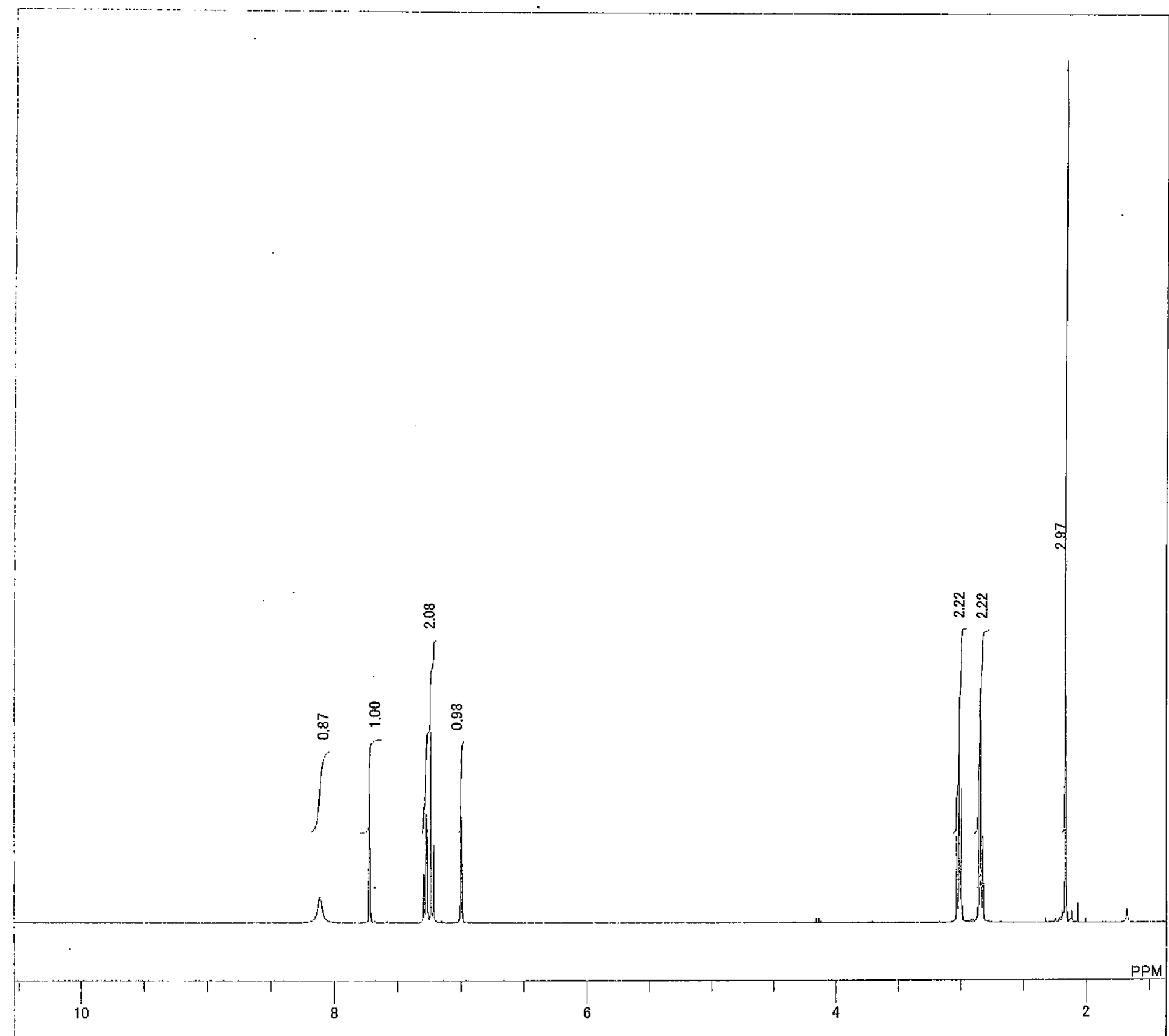

DFILE

DATIM

OBNUC

EXMOD

OBFRQ

OBSET

OBFIN

D

FREQU

SCANS

ACQT

PW1

IRNUC

CTEMP

RGAIN

Eca $¥ d a t a ¥ G u ¥ 335.1$

01-03-2006 15:05:36

gle_pulse.ex2
$399.78 \mathrm{MHz}$ $.19 \mathrm{KHz}$ $.29 \mathrm{~Hz}$

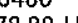

$9378.89 \mathrm{~Hz}$

$2.1837 \mathrm{sec}$ $2.1837 \mathrm{sec}$ 5.50 usec

$1 \mathrm{H}$

$\operatorname{CDCL}^{24.30}$

$7.24 \mathrm{ppm}$

$0.12 \mathrm{~Hz}$

4-(5-Bromo-3-indolyl)-2-butanone (2)

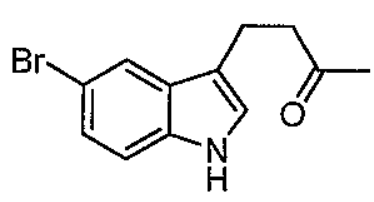


¥¥Eca $¥$ data $¥ G u ¥ 335-13 \mathrm{C} .1$

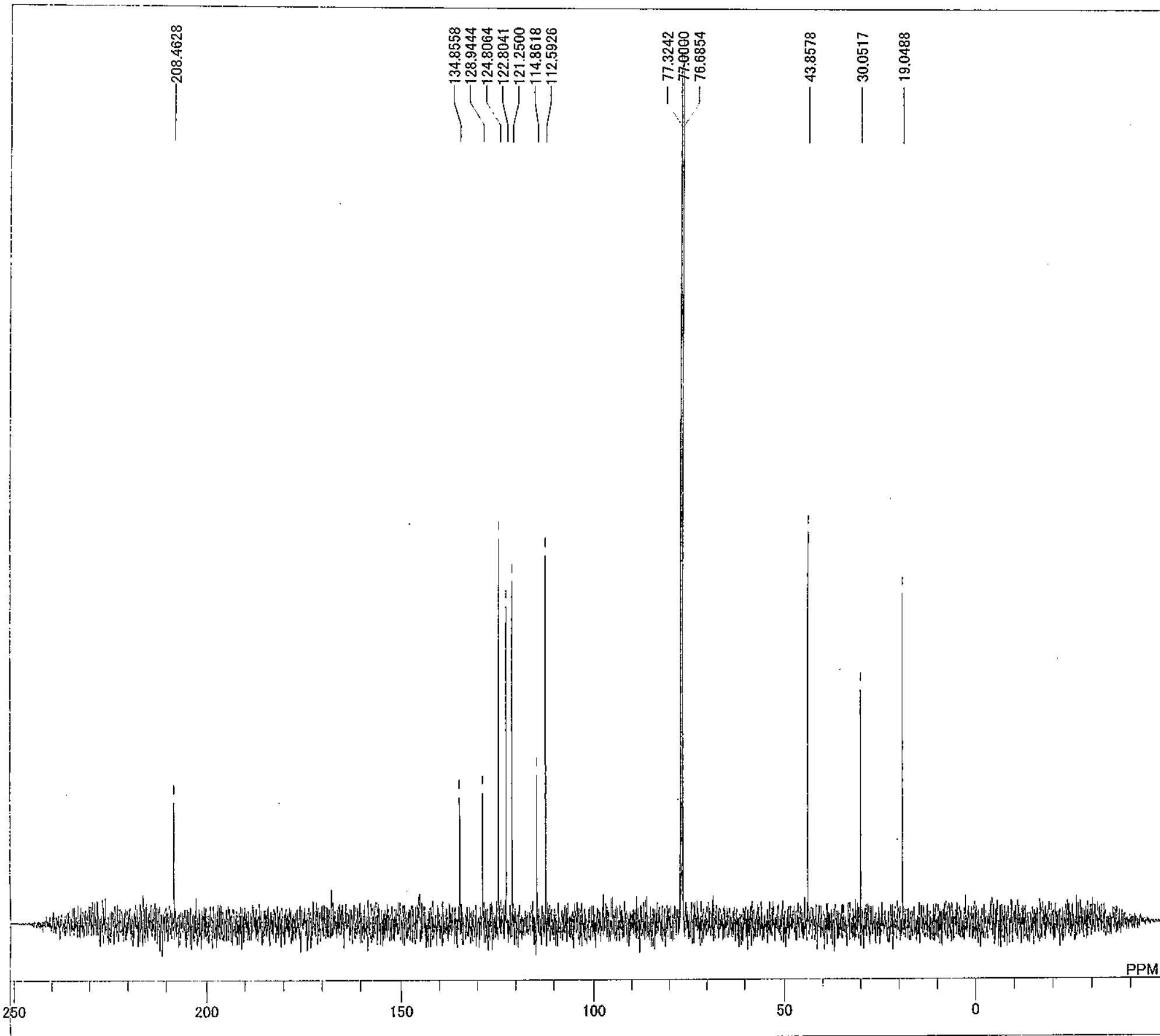

DFILE

COMNT

OBNUC

EXMOD

OBFRQ

OBSET

POINT

FREQU

SCANS

PCQTM

PD

IRNUC

CTEMP

SLVNT

EXREF

RF

$\operatorname{CDCL3}$

$77.00 \mathrm{ppm}$
$0.12 \mathrm{~Hz}$

56

4-(5-Bromo-3-indolyl)-2-butanone (2)

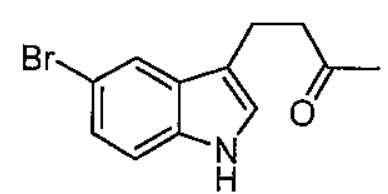




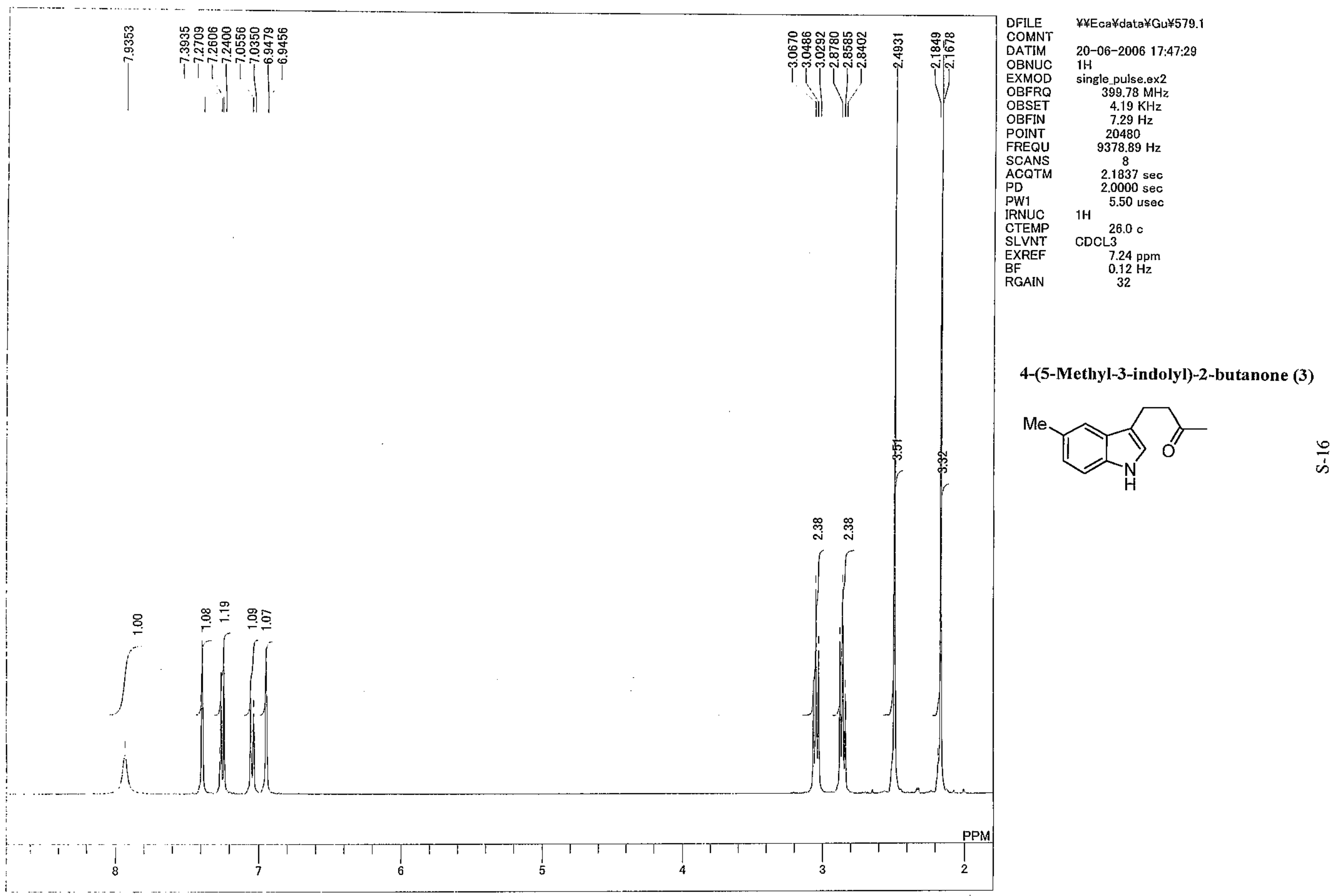




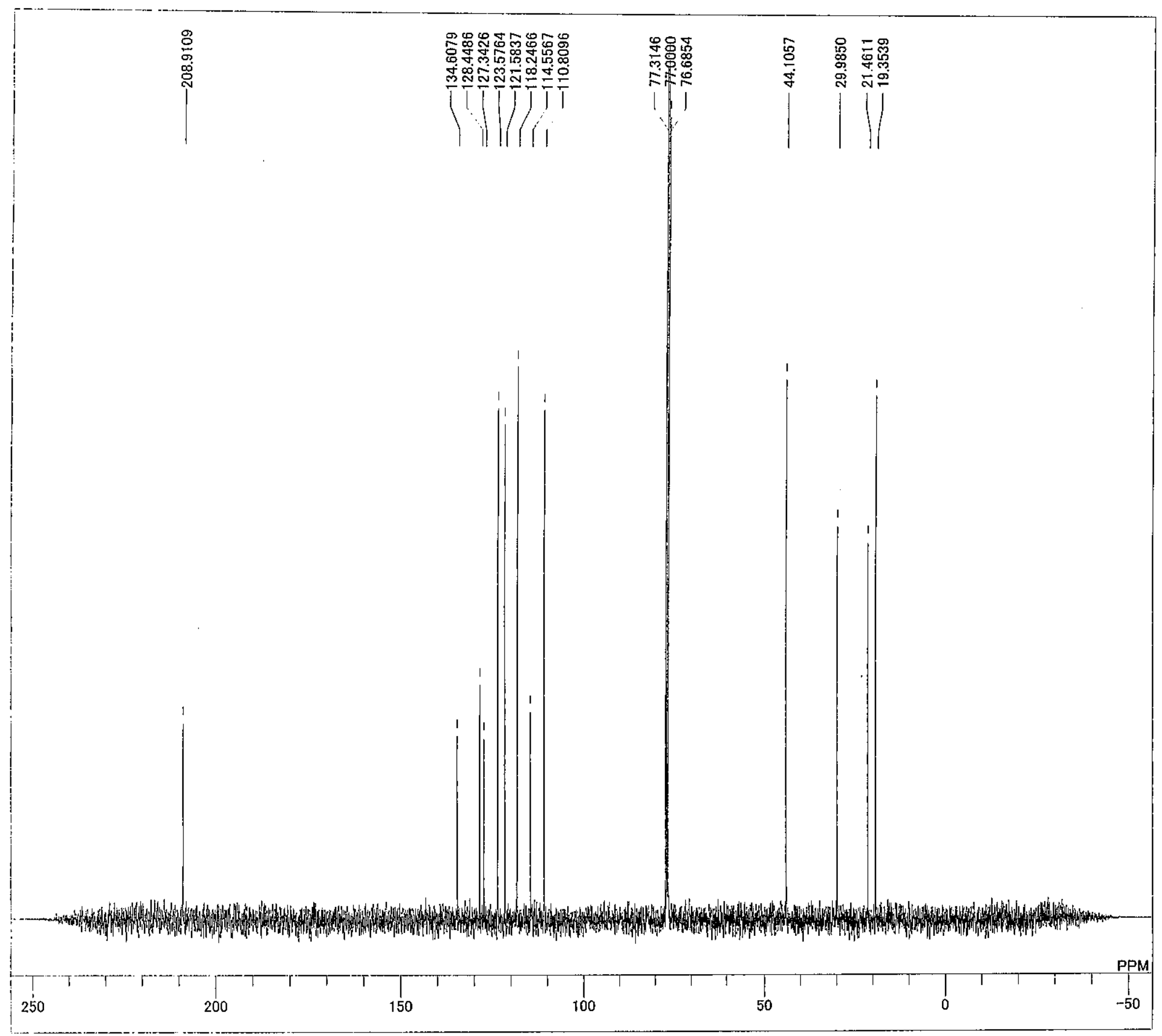

DFILE

DATIM

DATHO

EXMOD

OBSET

OBFIN

POINT

FREQU

SCANS

ACQTM

PD1

IRNUC

SLVNT

EXREF

BF

RGAIN

20-06-2006 17:54:31

ingle_pulse_dec

$5.35 \mathrm{KHz}$ $5.86 \mathrm{~Hz}$

40961

$39259.39 \mathrm{~Hz}$

118

$1.0433 \mathrm{sec}$

$2.0000 \mathrm{sec}$

$1 \mathrm{H}$

CDCL 3

$77.00 \mathrm{ppm}$

56

4-(5-Methyl-3-indolyl)-2-butanone (3)

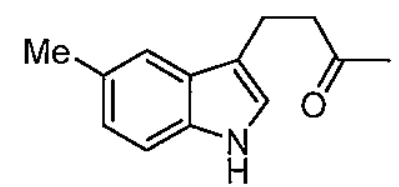

$\frac{1}{1}$ 


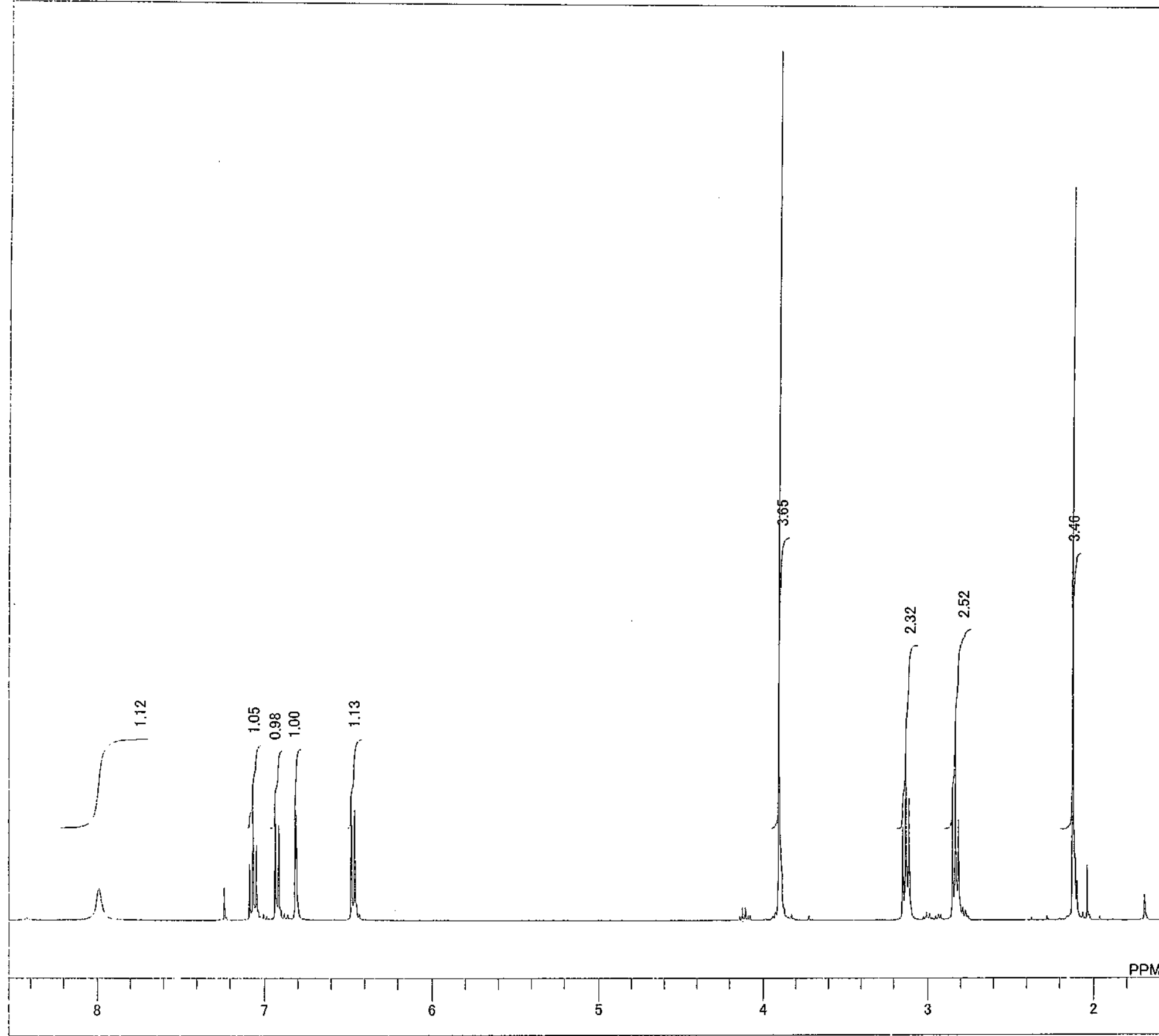

DFILE

DATIM

OBNUC

EXMOD

OBFRQ

OBSET

OBFIN

POINT

FREQU

SCANS

ACQTM

PD

IRNUC

CTEMP

SLVNT

EXREF

RGAIN

4-(4-Methyl-3-indolyl)-2-butanone (4)

$\frac{\infty}{n}$

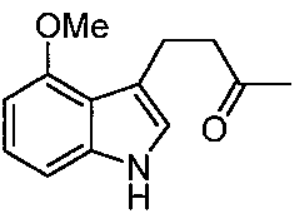

${ }_{32}^{0.12} \mathrm{~Hz}$

7YEca data:Gu¥329.1

2006 20:09:08

epulse.ex2

$4.19 \mathrm{KHz}$

20480

$9378.89 \mathrm{~Hz}$

$2.1837 \mathrm{sec}$

550 sec

2500

(5)

32

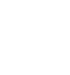


¥¥Eca data $¥ G u ¥ 329-13 \mathrm{C} .1$

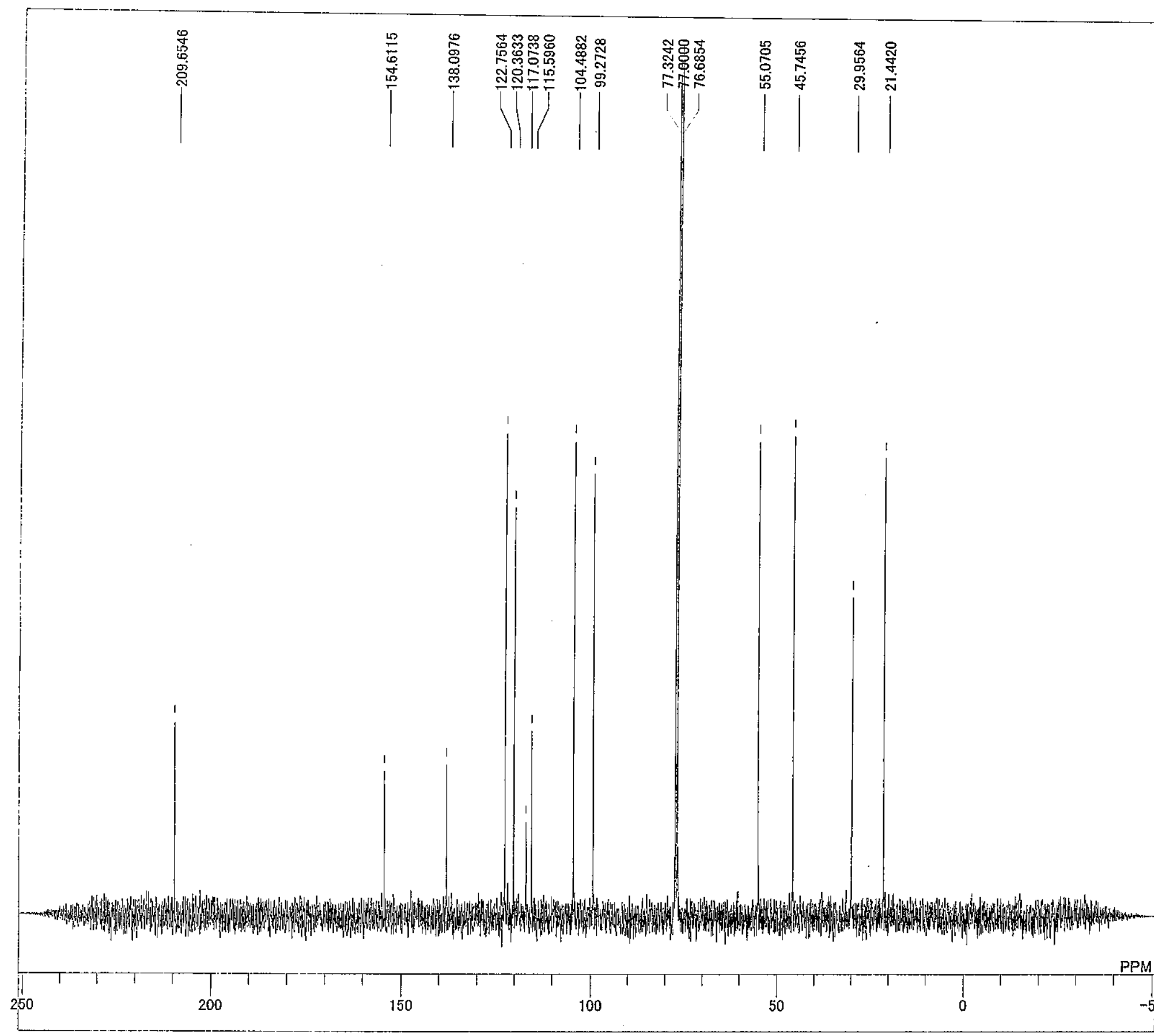

DFILE

OBFRQ

OBSET

OBFIN

POINT

FREQL

SCANS

PD

IRNUC

CTEMP

SLVNT

EXREF

RGAIN \#\#ca $¥$ data $¥ \mathrm{Gu} ¥ 329-13 \mathrm{C} .1$

24-02-2006 20:18:40

$100.53 \mathrm{MHz}$ $5.35 \mathrm{KHz}$ $5.86 \mathrm{~Hz}$ 40961 $39259.39 \mathrm{~Hz}$ 90 $1.0433 \mathrm{sec}$ $2.0000 \mathrm{sec}$ 3.33 usec $\operatorname{CDCL} 3.3$ $77.00 \mathrm{ppm}$ 56

음

4-(4-Methyl-3-indolyl)-2-butanone (4)<smiles>COc1cccc2[nH]cc(CCC(C)=O)c12</smiles> 


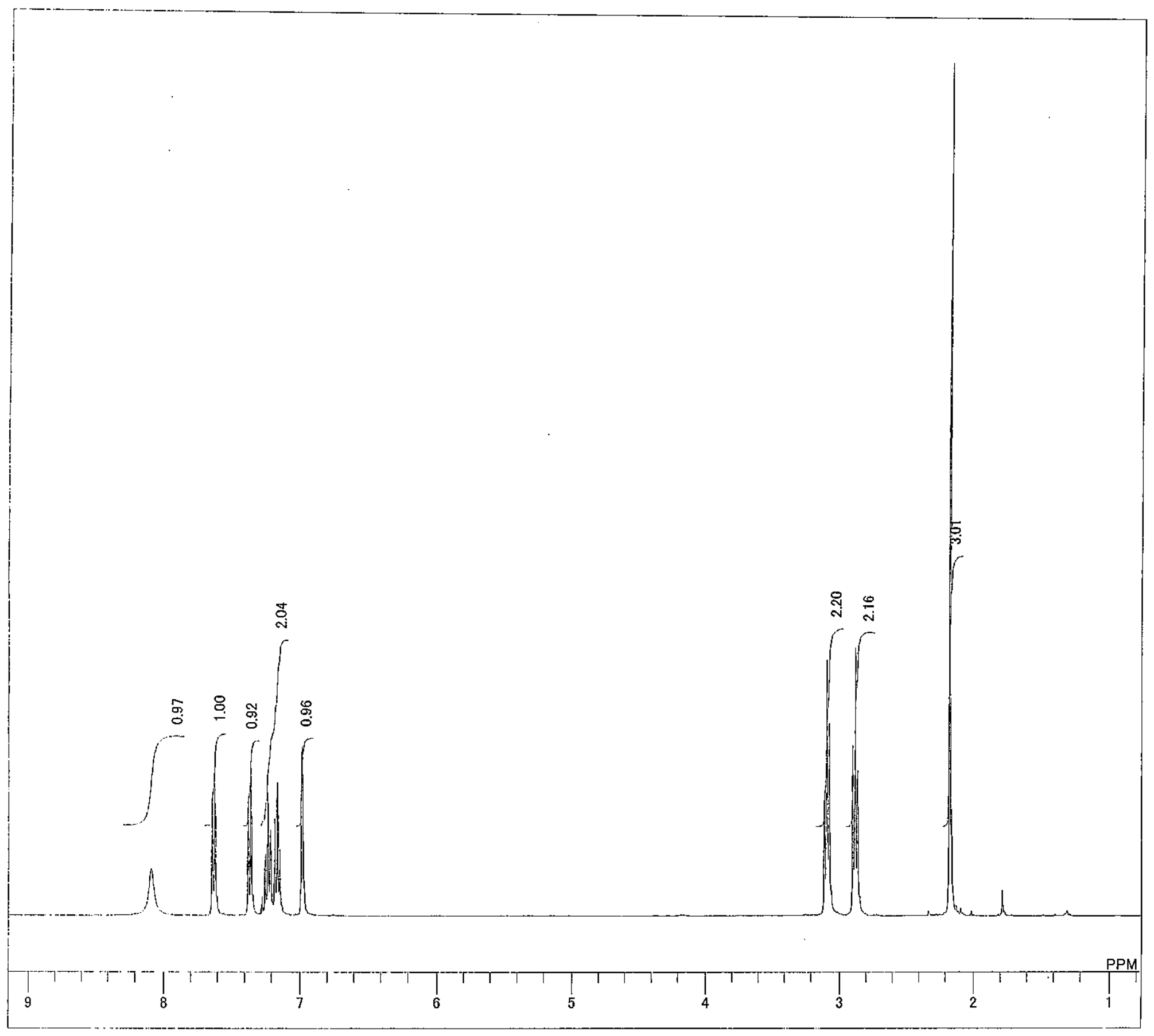

OBNUC

EXMOD

OBFRQ

OBSET

OBFIN

POINT

FREQU

SCANS

PD

IRNUC

CTEMP

SLVNT

EXRE

RGAIN

$\operatorname{CDCL} 3$

$1.20 \mathrm{pz}$

28

4-(1-1 $H$-3-Indolyl)-2-butanone (5)

ซิ

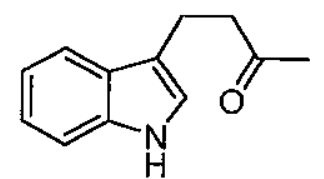


¥¥Eca $¥$ data $¥ G u ¥ 320-13 \mathrm{C}, 1$

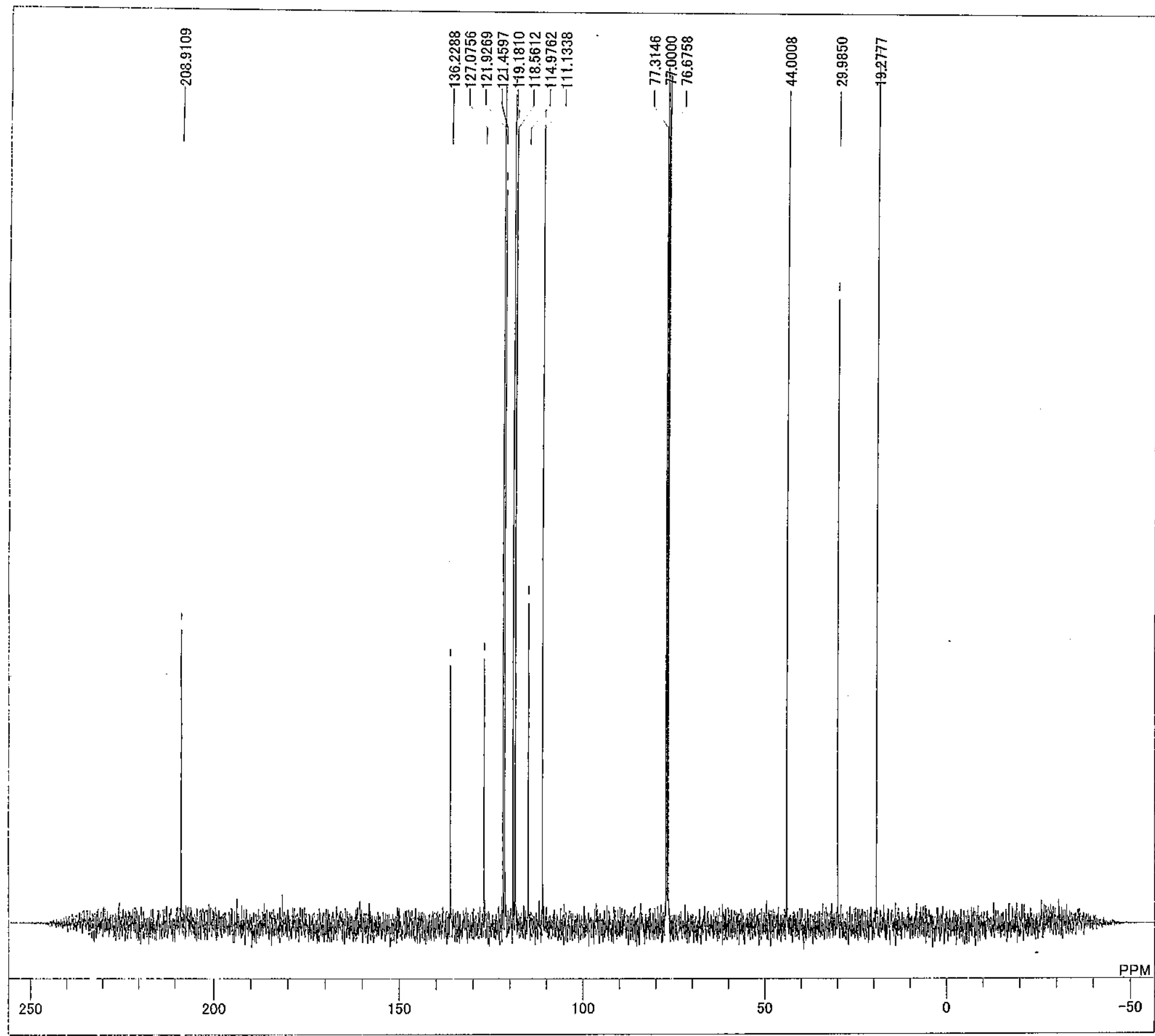

DATIM

EXMOD

EXMOD

OBSET

OBFIN

POINT

FREQU

SCANS

ACQTM

PW1

IRNUC

CTEMP

SLVNT

EXREF

RGAIN

$¥ E c a ¥ d a t a ¥ G u ¥ 320-13 \mathrm{C} .1$

16-02-2006 20:53:53

ingle_pulse_dec $100.53 \mathrm{MHz}$ $5.86 \mathrm{~Hz}$

40961

$39259.39 \mathrm{~Hz}$

113

$1.0433 \mathrm{sec}$
$2.0000 \mathrm{sec}$

3.33 usec

$\operatorname{CDCL}^{24.0 \circ}$

$77.00 \mathrm{ppm}$

$1.20 \mathrm{~Hz}$
56

4-(1-1H-3-Indolyl)-2-butanone (5)

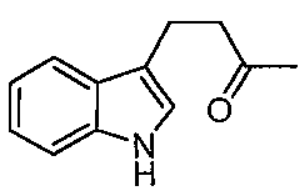


OBNUC

02-03-2006 09:08:04

EXMOD

single pulse.ex2

$399.78 \mathrm{MHz}$

OBSEN

PBFIN

$7.29 \mathrm{~Hz}$

16384

FREQU $\quad 7503.00 \mathrm{~Hz}$

SCANS

ACQTM

PD

8
$2.1837 \mathrm{sec}$

$2.1837 \mathrm{sec}$
$2.0000 \mathrm{sec}$

IRNUC $\quad \begin{aligned} & 2.50 \text { usec } \\ & \text { PH }\end{aligned}$

CTEMP

CTEMP

23.7

EXREF

$7.24 \mathrm{ppm}$

RGAIN

$7.24 \mathrm{ppm}$
$0.12 \mathrm{~Hz}$
32

(2)

4-(5-Methoxy-3-indolyl)-2-butanone (6)

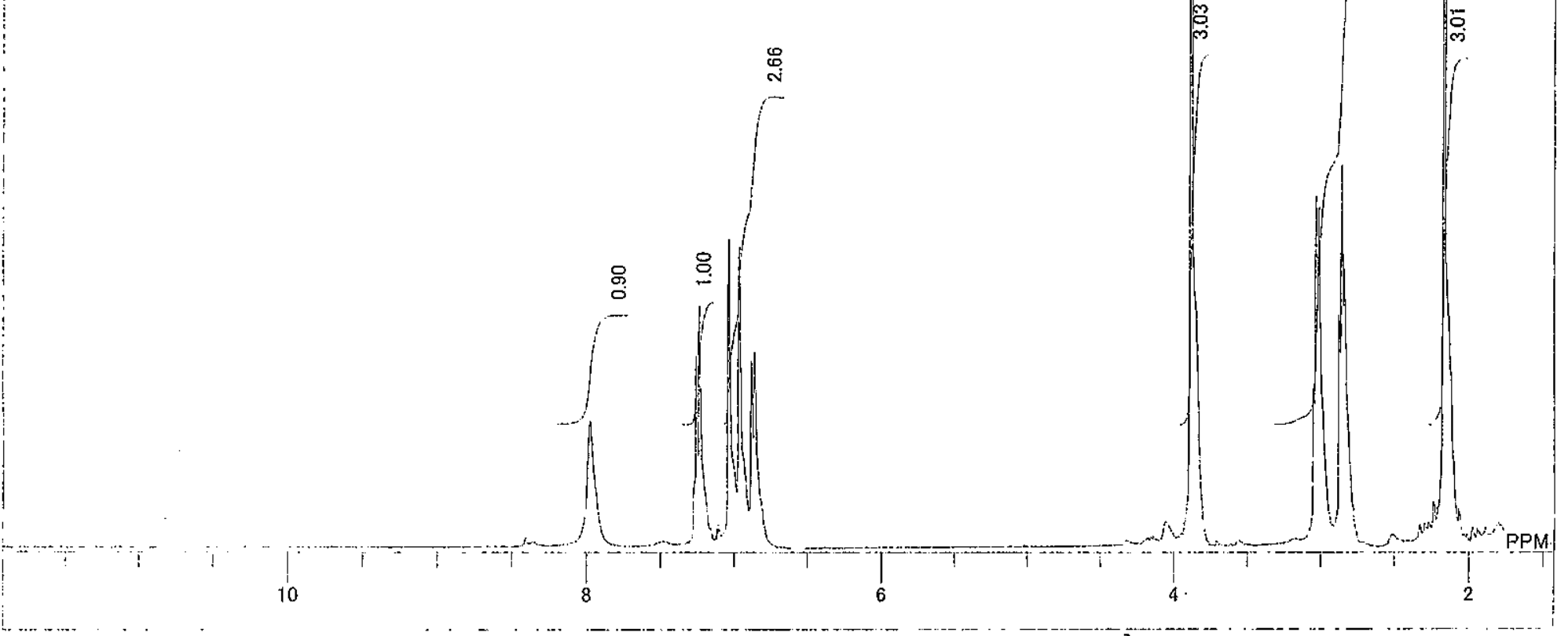


¥¥Eca data $¥ 334-13 \mathrm{C} .1$

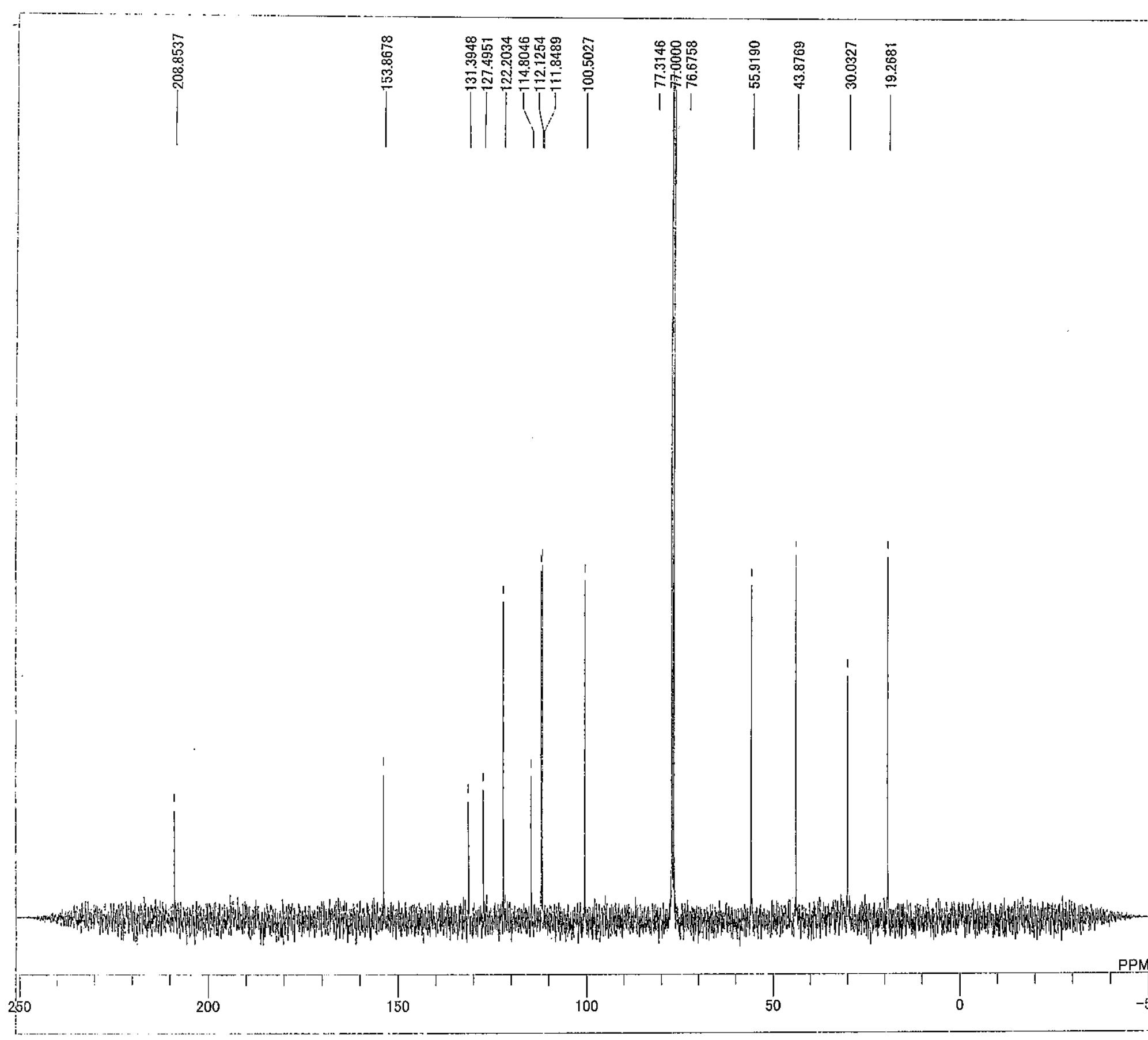

DFIL.E

EXMOD

OBFRQ

OBSET

OBFIN

POINT

FREQU

SCANS

ACQTM

PI

IRNUC

CTEMP

SLVNT

EXREF

RGAIN

$\mathrm{CDCL}$

$77.00 \mathrm{ppm}$

50

4-(5-Methoxy-3-indolyl)-2-butanone (6)$$
\text { MeOr }
$$

$\mathrm{MeO}$

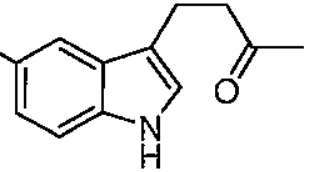




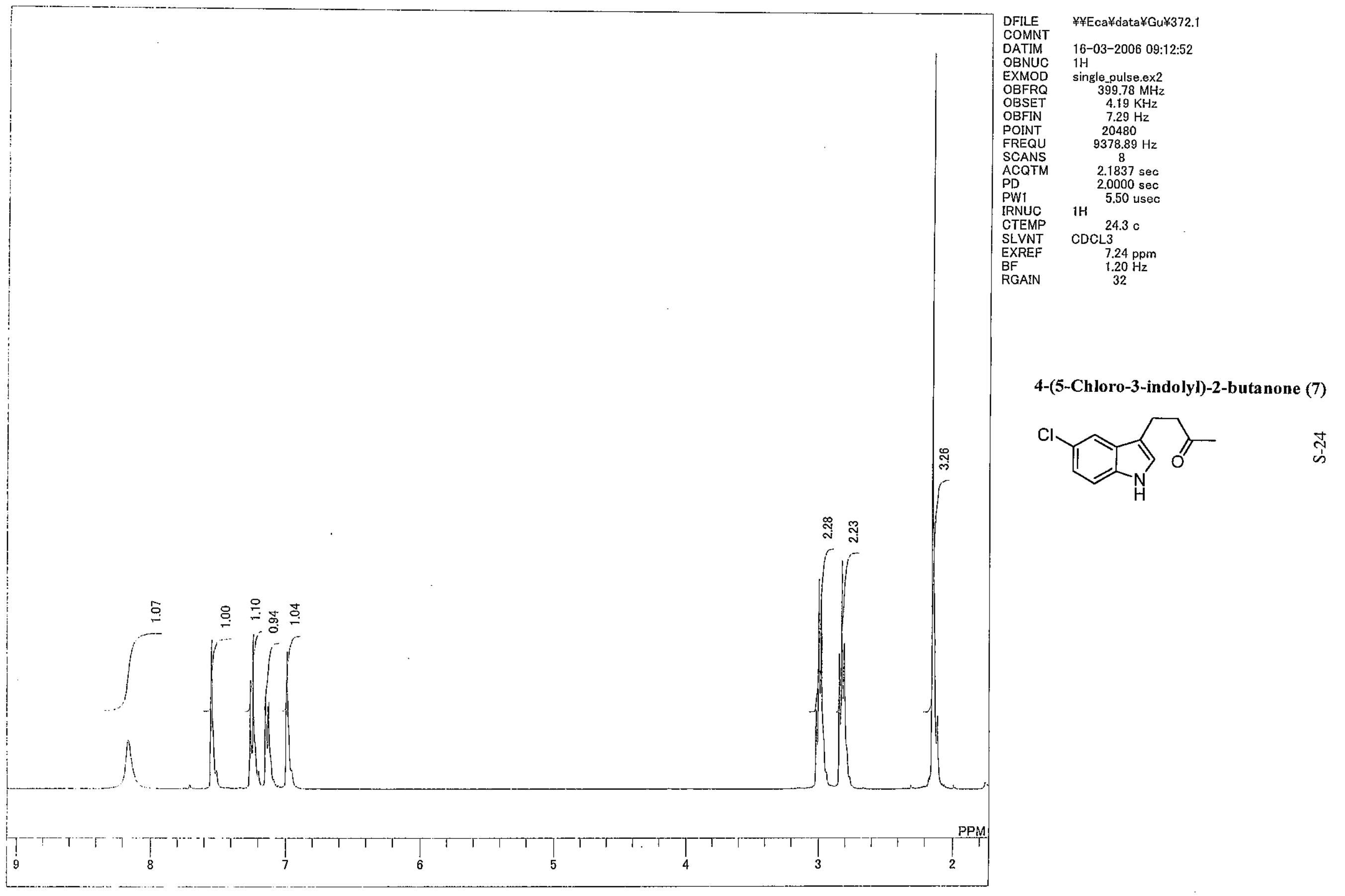




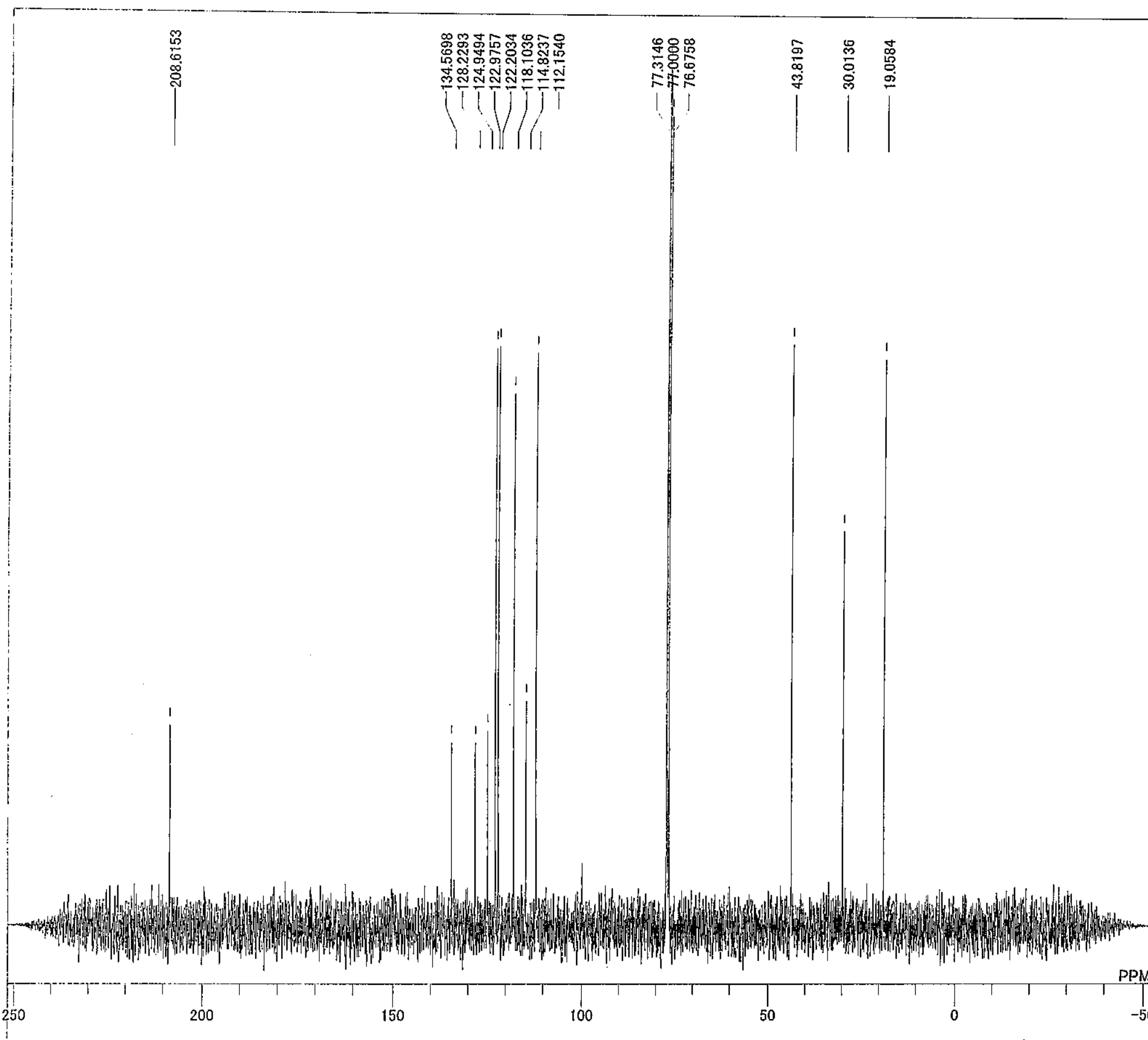

DFILE

DATIM

OBNUC

EXMOD

OBSET

OBFIN

POINT

FREQU

SCANS

ACQTM

PW1

IRNUC

CTEMP

SLVNT

EXREF

BF

RGAIN

$\operatorname{CDCL} 3$

$77.00 \mathrm{ppm}$

56

4-(5-Chloro-3-indolyl)-2-butanone (7)

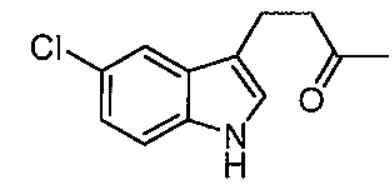




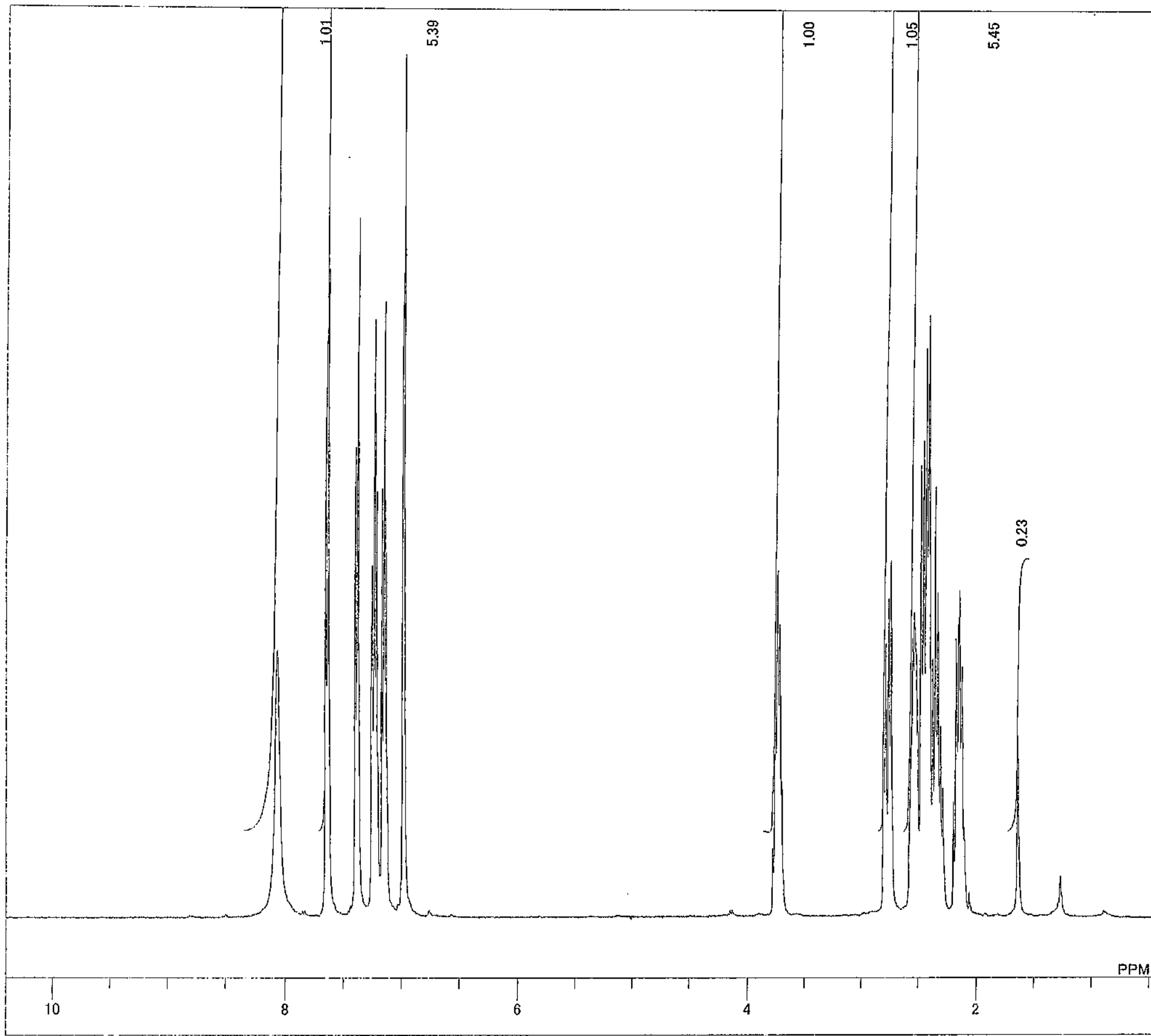

DFILE DOMNT

DATIM

OBNUC

OBFRQ

OBSET

OBFIN

POINT

FREQU

SCANS

ACQTM

PD

PW1

IRNUC

SLVNT

EXREF

RGAIN
$¥ ¥ \mathrm{Eca}$ data $¥ \mathrm{Gu} ¥ 323.1$

1-02-2006 13:43:26

gle_pulse.ex2

$4.78 \mathrm{MHz}$

$7.29 \mathrm{~Hz}$

20480

$9378.89 \mathrm{~Hz}$

8
$2.1837 \mathrm{sec}$

$2.0000 \mathrm{sec}$

5.50 usec
$1 \mathrm{H}$

25.1

$7.24 \mathrm{ppm}$

$7.24 \mathrm{ppm}$
$0.12 \mathrm{~Hz}$

34

3-(1-1H-3-Indolyl)-cyclopen tanone (8)

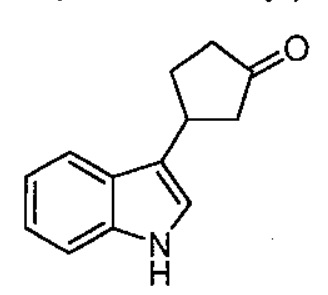


¥¥Eca đdata $¥ \mathrm{G} u ¥ 323-13 \dot{\mathrm{C}} .1$

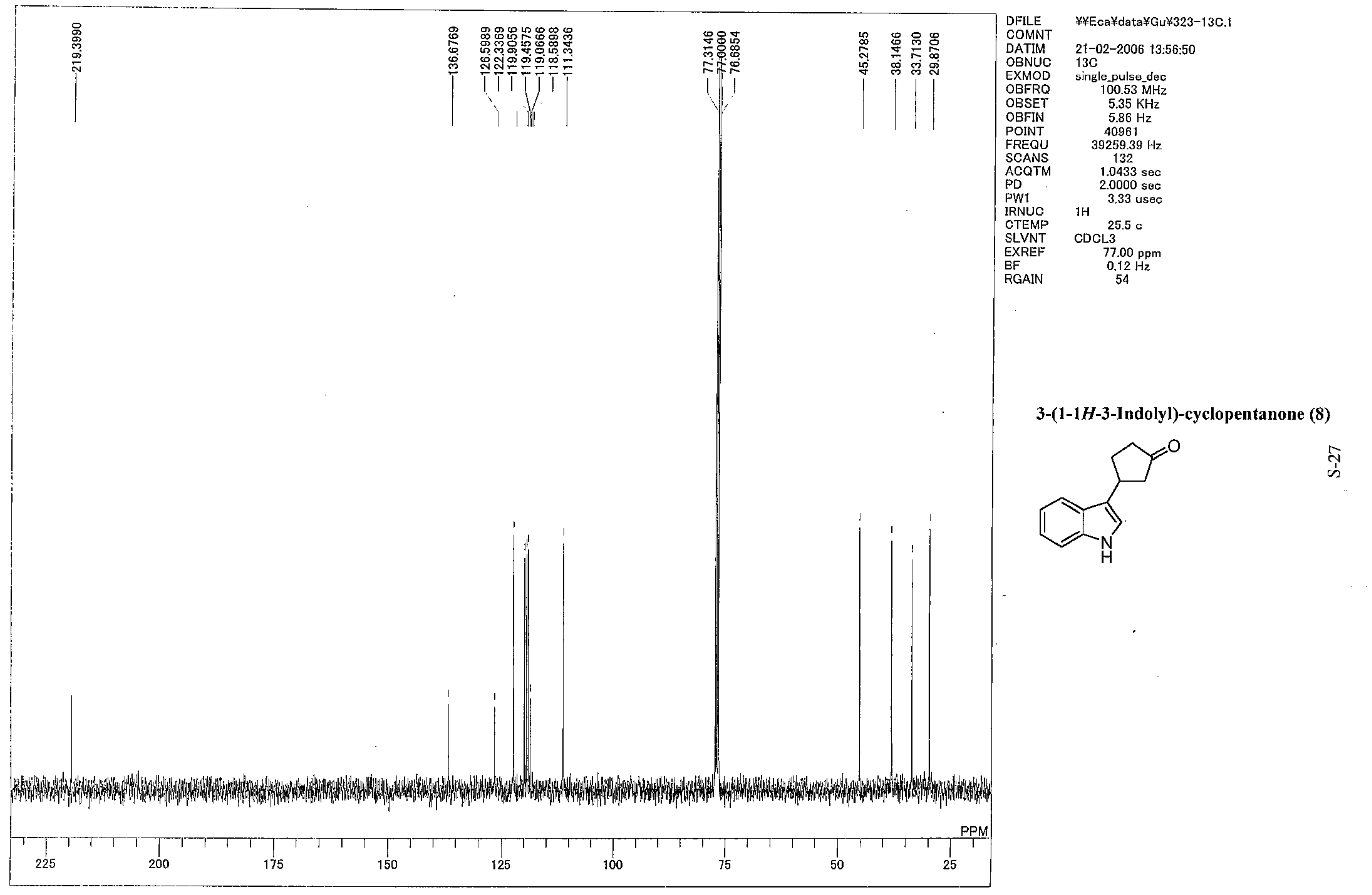




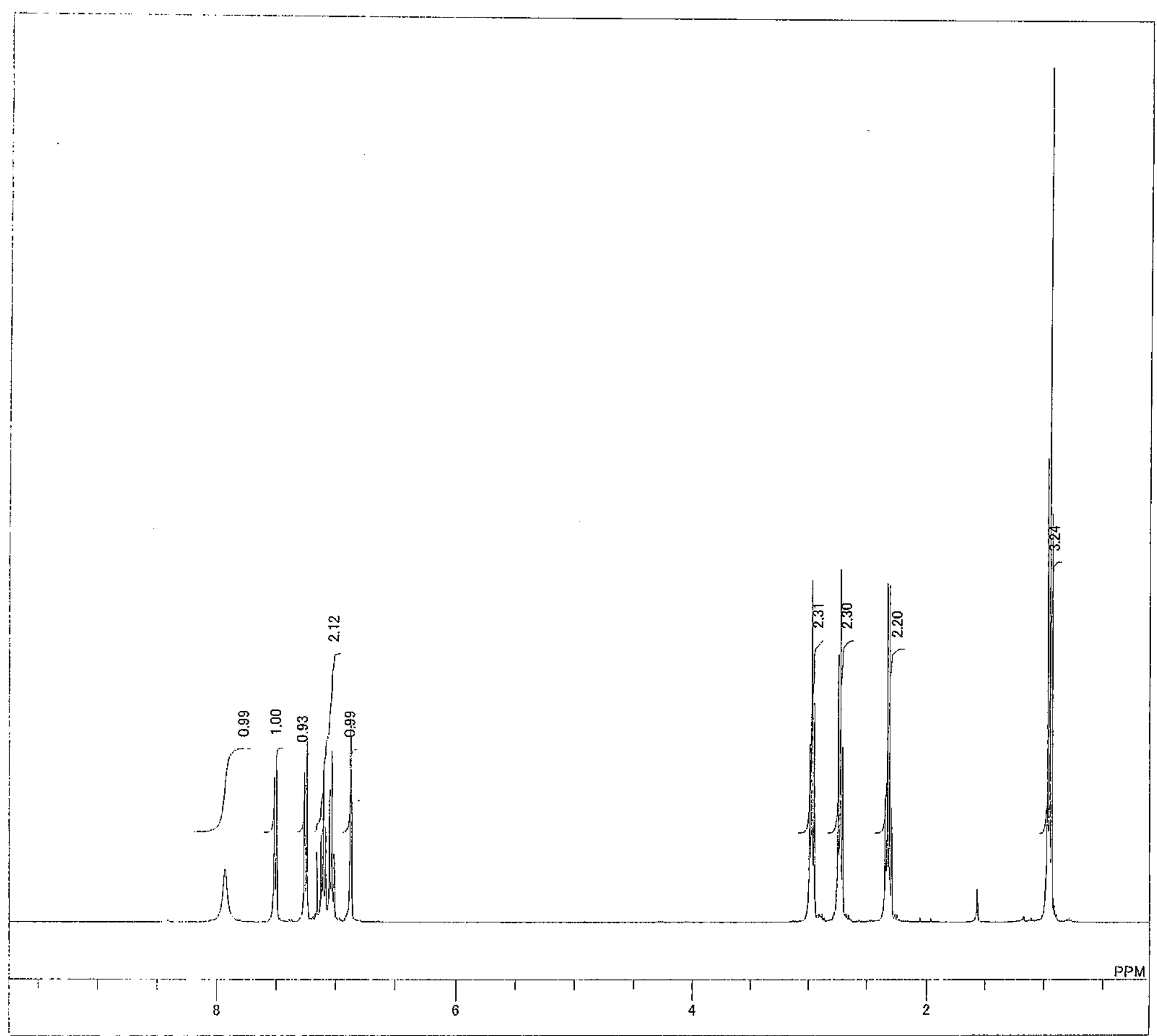

DFILE

COMNT

DATIM

EXMOD

EXFRQ

OBSET

OBFIN

POINT

FREQU

SCANS

ACQTM

PD

IRNUC

CTEMP

SLVNT

EXREF

BF

$¥ ¥ \mathrm{Eca} \approx$ data $¥ \mathrm{G} \mathrm{u} ¥ 340.1$

02-03-2006 18:10:51

single_pulse.ex2 $399.78 \mathrm{MHz}$
$4.19 \mathrm{KHz}$ $7.29 \mathrm{~Hz}$

20480

$9378.89 \mathrm{~Hz}$

8
$2.1837 \mathrm{sec}$

5.50 usec

IH $\quad 5.50$ usec

CDCL3

$0.00 \mathrm{ppm}$
$0.12 \mathrm{~Hz}$

$0.12 \mathrm{~Hz}$
32

1-(1 H-Indol-3-yl)-pentan-3-one (9)

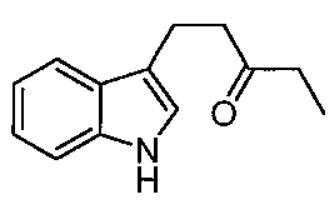




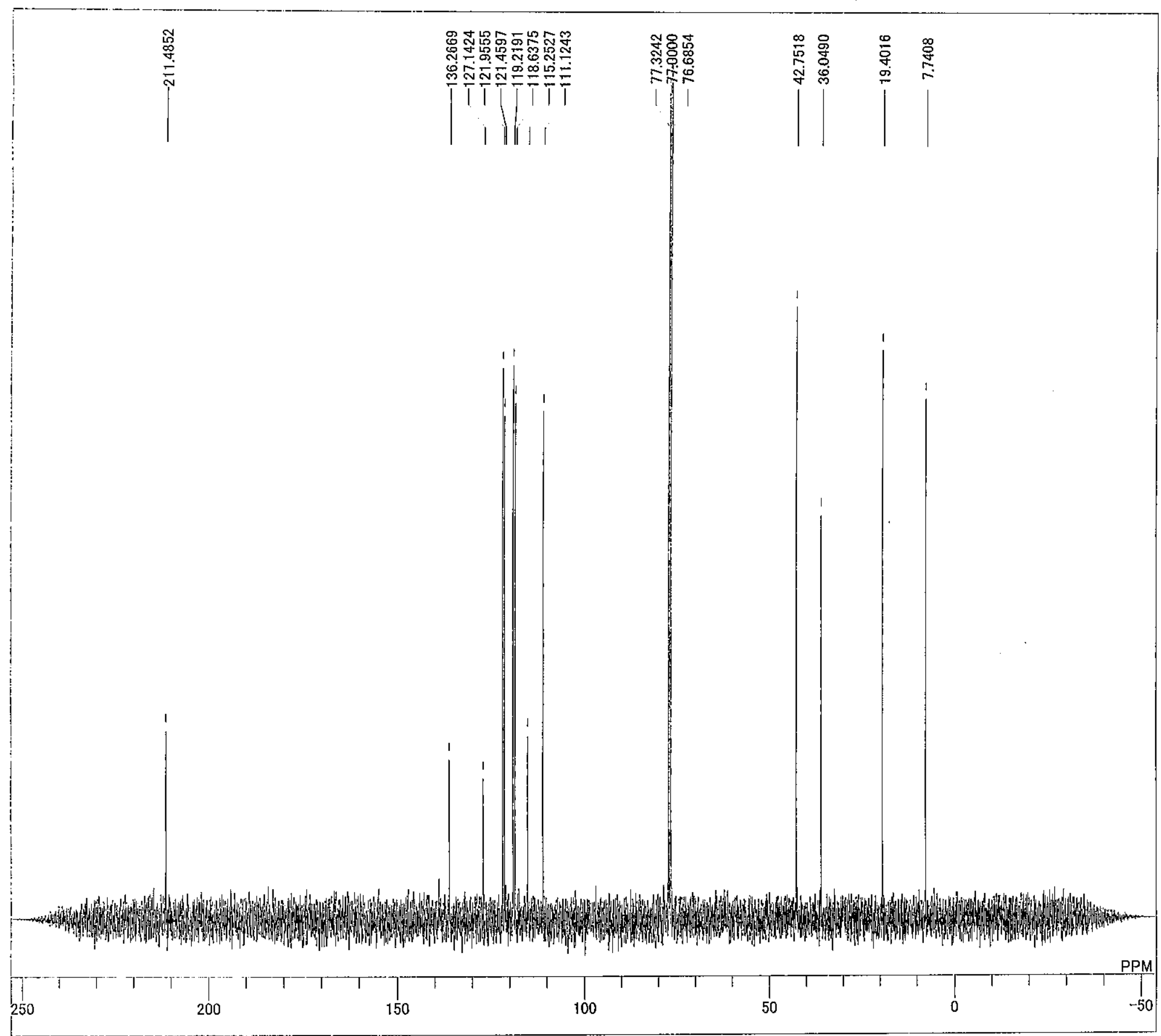

DFILE

DATIM

EXMOD

OBFRQ
OBSET

OBSET
OBFIN

POINT

FREQU

ACQTM

$\mathrm{PD}$

PW1

CTEMP

SLVNT

EXREF

RGAIN

$¥ ¥ E$ ca $¥$ data $¥ G u ¥ 340-13 C .1$

02-03-2006 18:15:03

single pulse dec

$100.53 \mathrm{MHz}$
$5.35 \mathrm{KHz}$

$5.86 \mathrm{~Hz}$ 40961

$39259.39 \mathrm{~Hz}$

59

$1.0433 \mathrm{sec}$

$2.0000 \mathrm{sec}$

1H 3.33 usec

CDCL 3.6

$77.00 \mathrm{ppm}$
$0.12 \mathrm{~Hz}$

1-(1H-Indol-3-yl)-pentan-3-one (9)

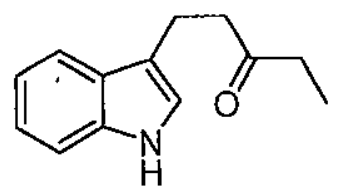


¥¥Eca $¥$ data $¥ G u ¥ 345.1$

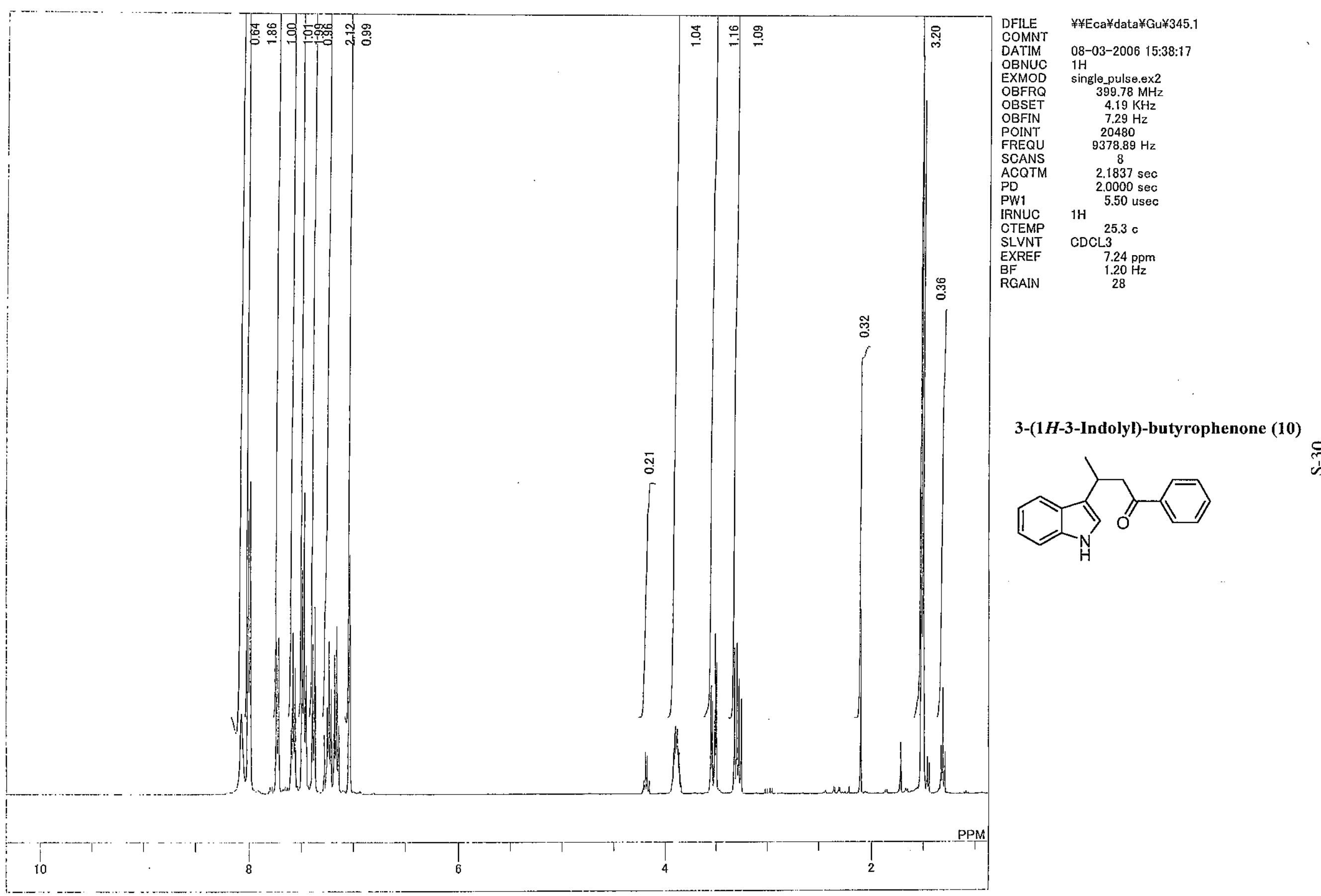


¥\#Eca $¥$ data $¥ G$ G $¥ 345-13 \mathrm{C} .1$

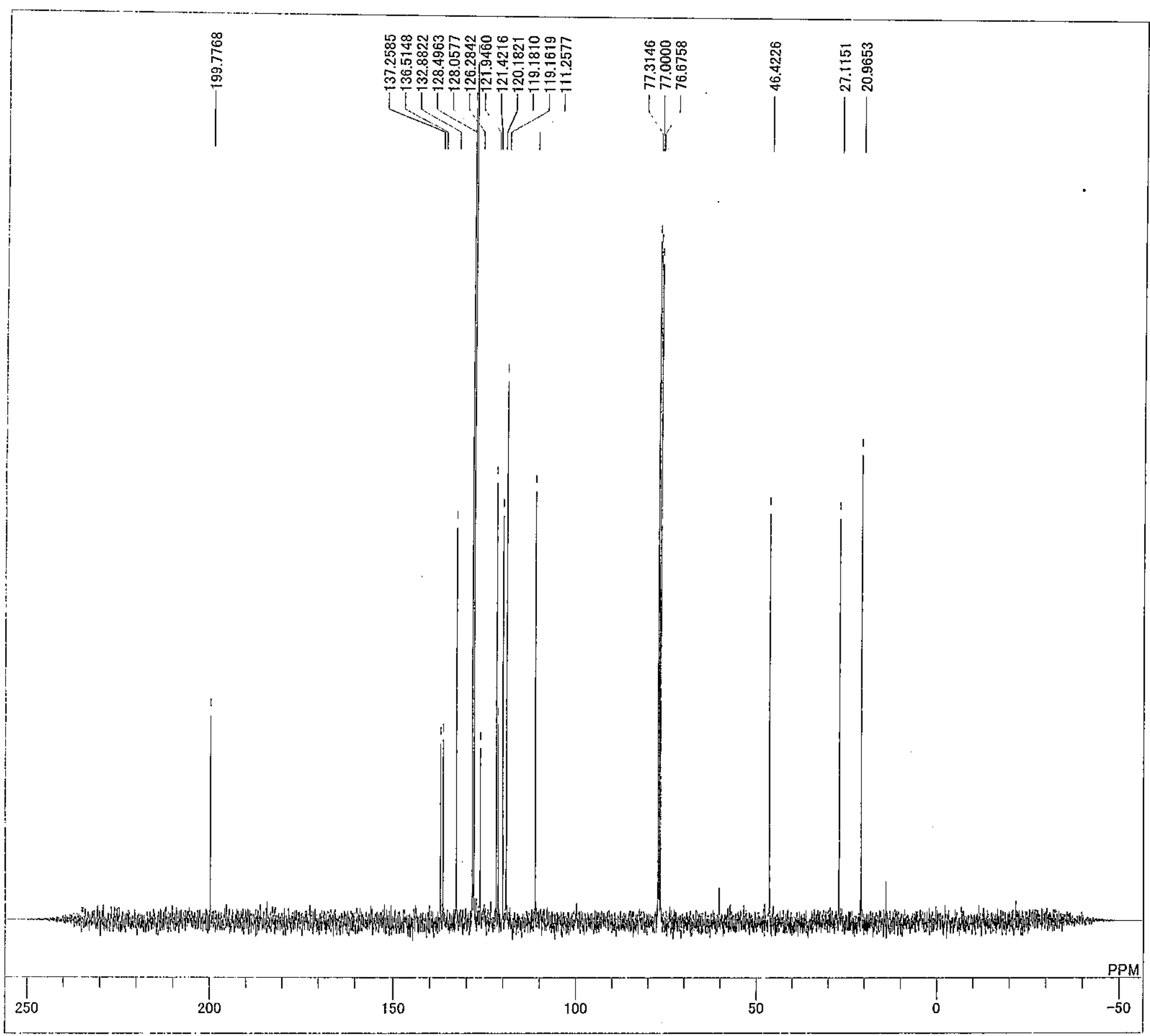

DFILE

COMN

EXMOD

OBFRQ

OBSET

OBFIN

POINT
FREQU

FREQU

ACQTM

PD

IRNUC

CTEMP

SL.VNT

EXREF

RGAIN

77.00

$1.20 \mathrm{H}$

3-(1 H-3-Indolyl)-butyrophenone (10)<smiles>CC(CC(=O)c1ccccc1)c1c[nH]c2ccccc12</smiles> 


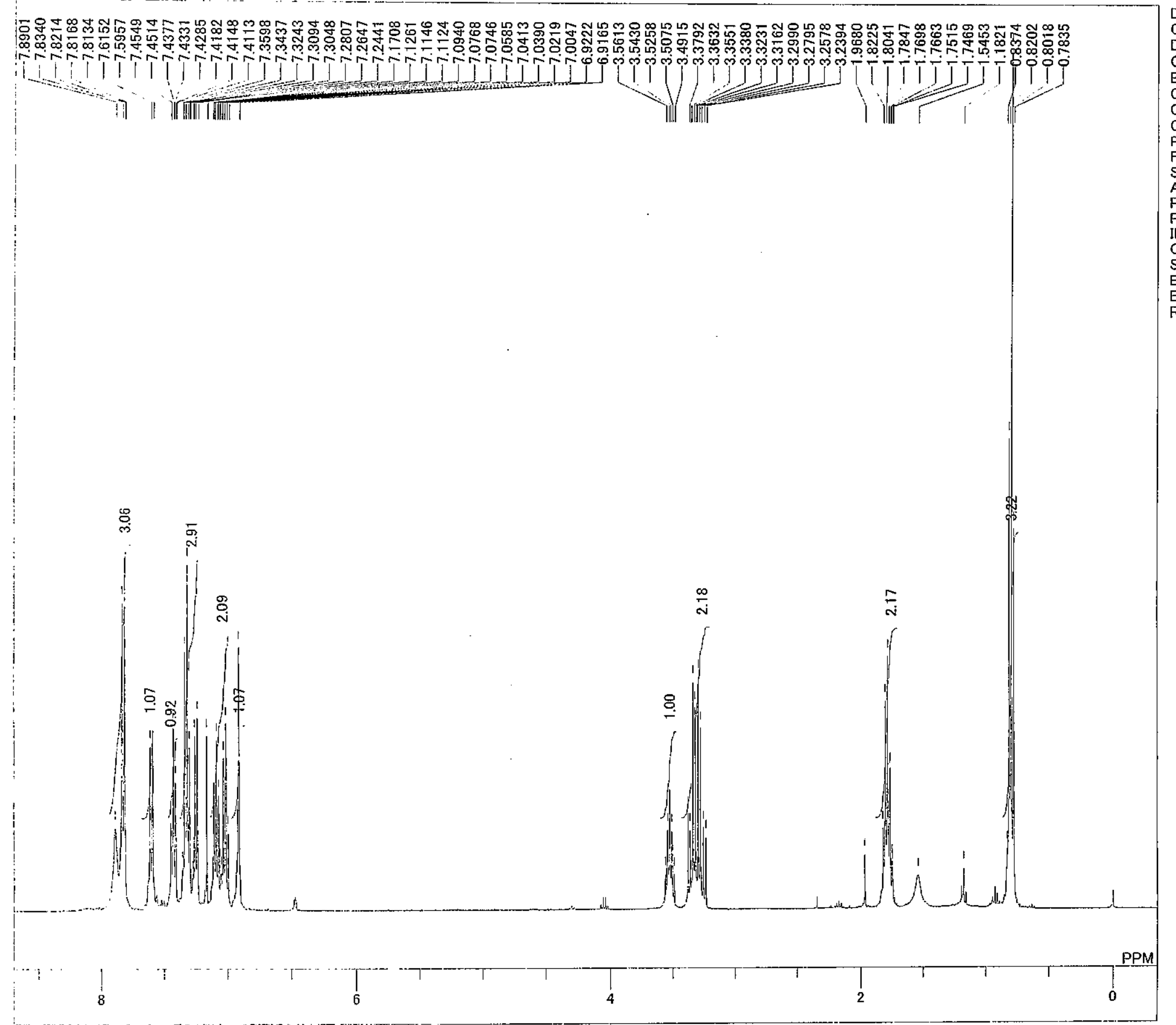

DFILE

COMNT

OBNUC

BFRO

OBSET

BFIN

SCANS

ACQTM

PD

IRNUC

CTEMP

SLVNT

EXREF

RGAIN

$¥ ¥ \mathrm{Eca} \approx$ data $¥ \mathrm{Gu} ¥ 539.1$

06-06-2006 16:33:48

gle_pulse.ex2 $3.78 \mathrm{MHz}$
$4.19 \mathrm{KHz}$ $7.29 \mathrm{~Hz}$ $9378.89 \mathrm{~Hz}$ 8

$2.1837 \mathrm{sec}$

$2.0000 \mathrm{sec}$

$1 \mathrm{H}$.

CDCL3

$0.00 \mathrm{ppm}$
$0.12 \mathrm{~Hz}$

34

3-(1H-3-Indolyl)-valerophenone (11)

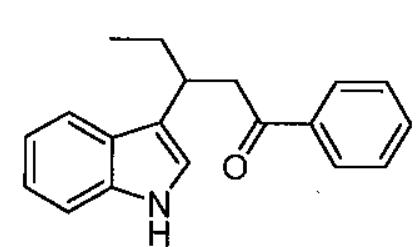


秏 $\mathrm{ca} ¥$ data $¥ \mathrm{Gu} ¥ 539-13 \mathrm{C} .1$

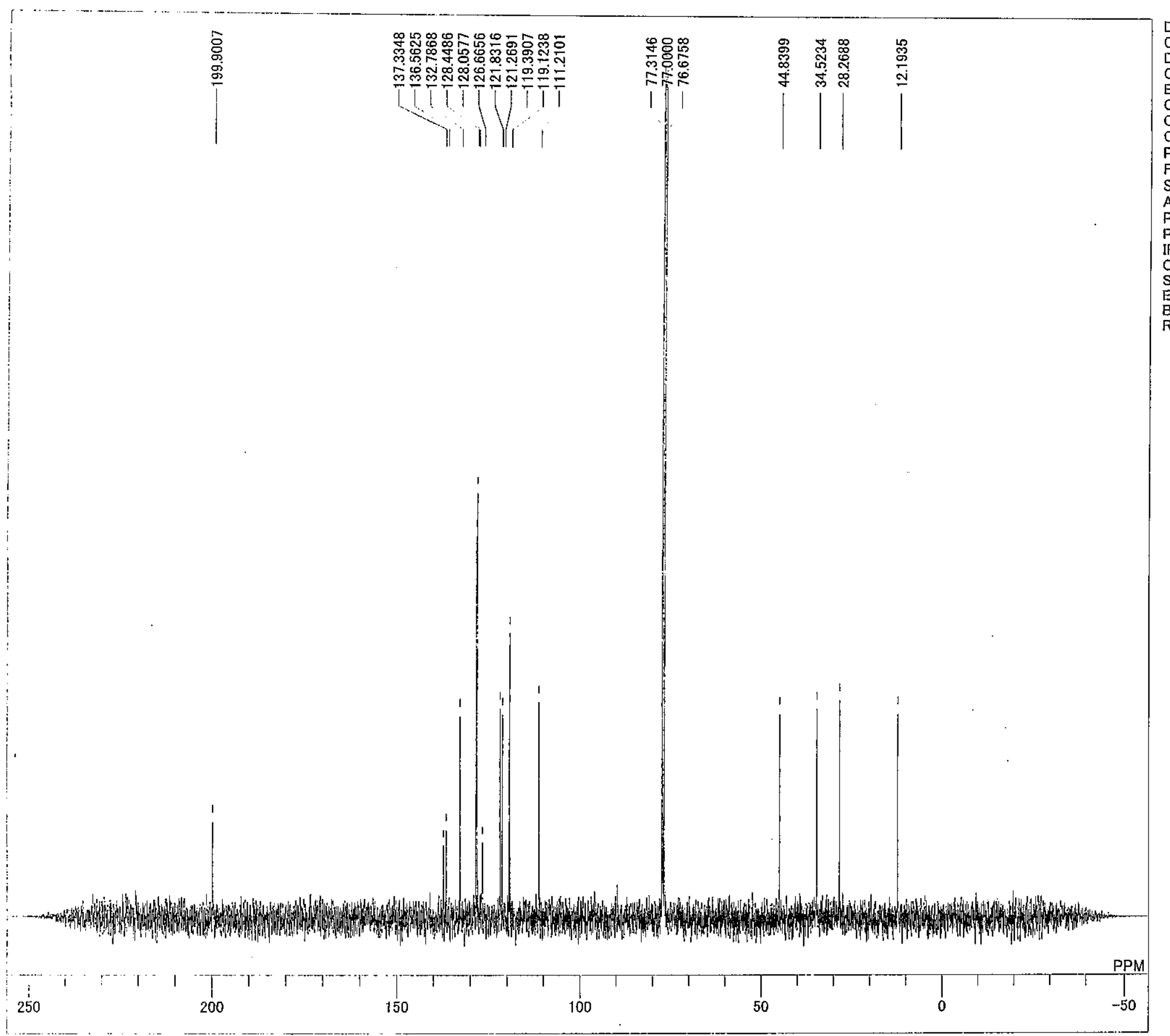

DFILE

DATIM

OBNUC

OBFRQ

OBSET

OBFIN

FREQU

SCANS

ACQTM

PD

IRNUC

CTEMP

XREF

F

RGAIN

$\operatorname{CDCL}_{77}^{25.8 \mathrm{C}}$

$77.00 \mathrm{ppm}$
$0.12 \mathrm{~Hz}$

56

3-(1H-3-Indolyl)-valerophenone (11)<smiles>CCC(CC(=O)c1ccccc1)c1c[nH]c2ccccc12</smiles> 
粍 $\mathrm{ca} ¥$ data $¥ G u ¥ 558.1$

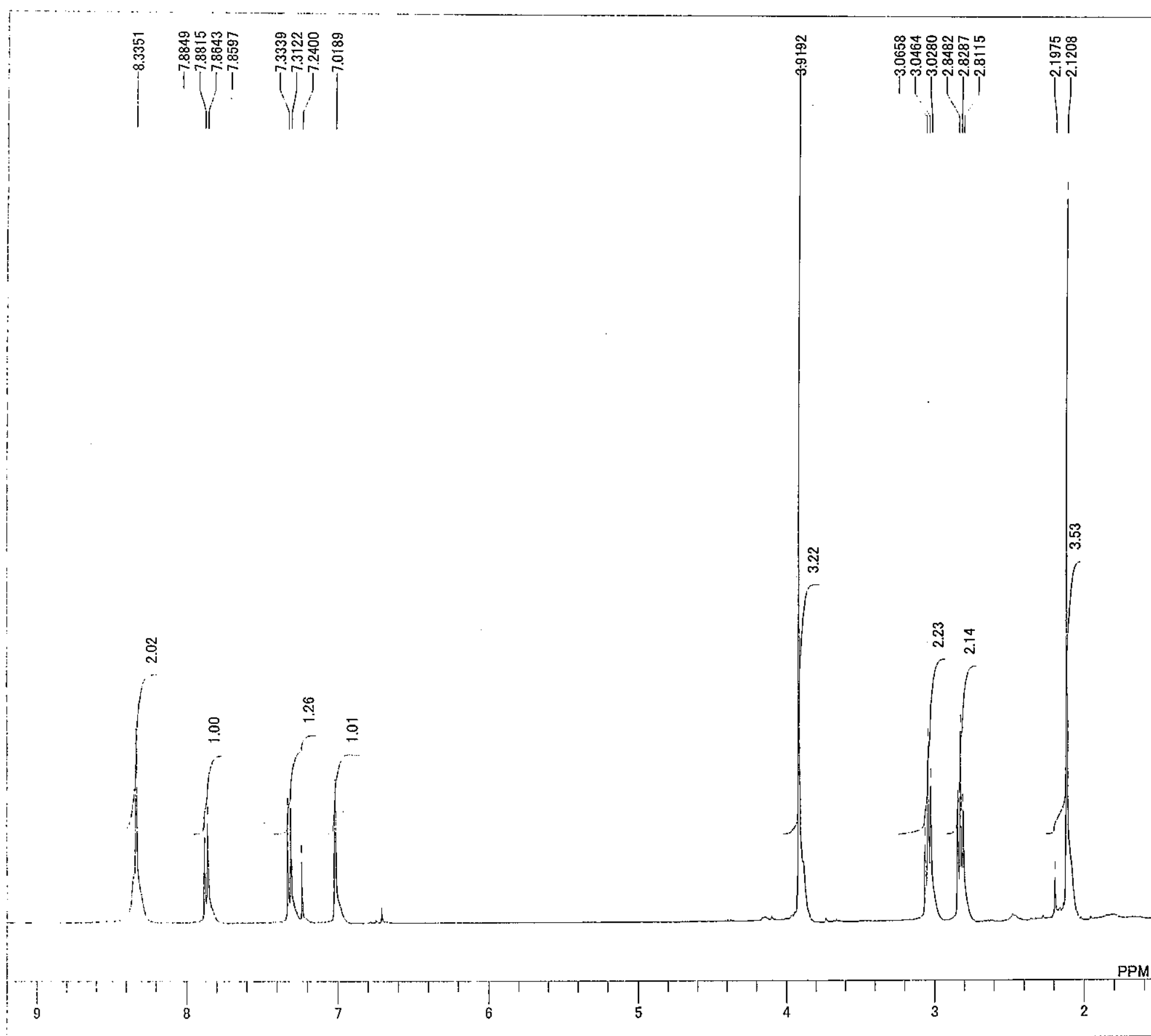

DFILE

DATIM

OBNUC

EXMOD

OBFRQ

OBSET

OBFIN

POINT

FREQU

SCANS

ACQTM

PD
PW1
IRNUC

IRNUC

SLVNT

RF

$\mathrm{CDCL}^{25.9}$

$7.24 \mathrm{ppm}$

34

Methyl 3-(3-oxobutyl)indole-5-carboxylate (12)

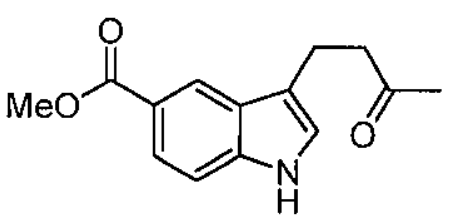




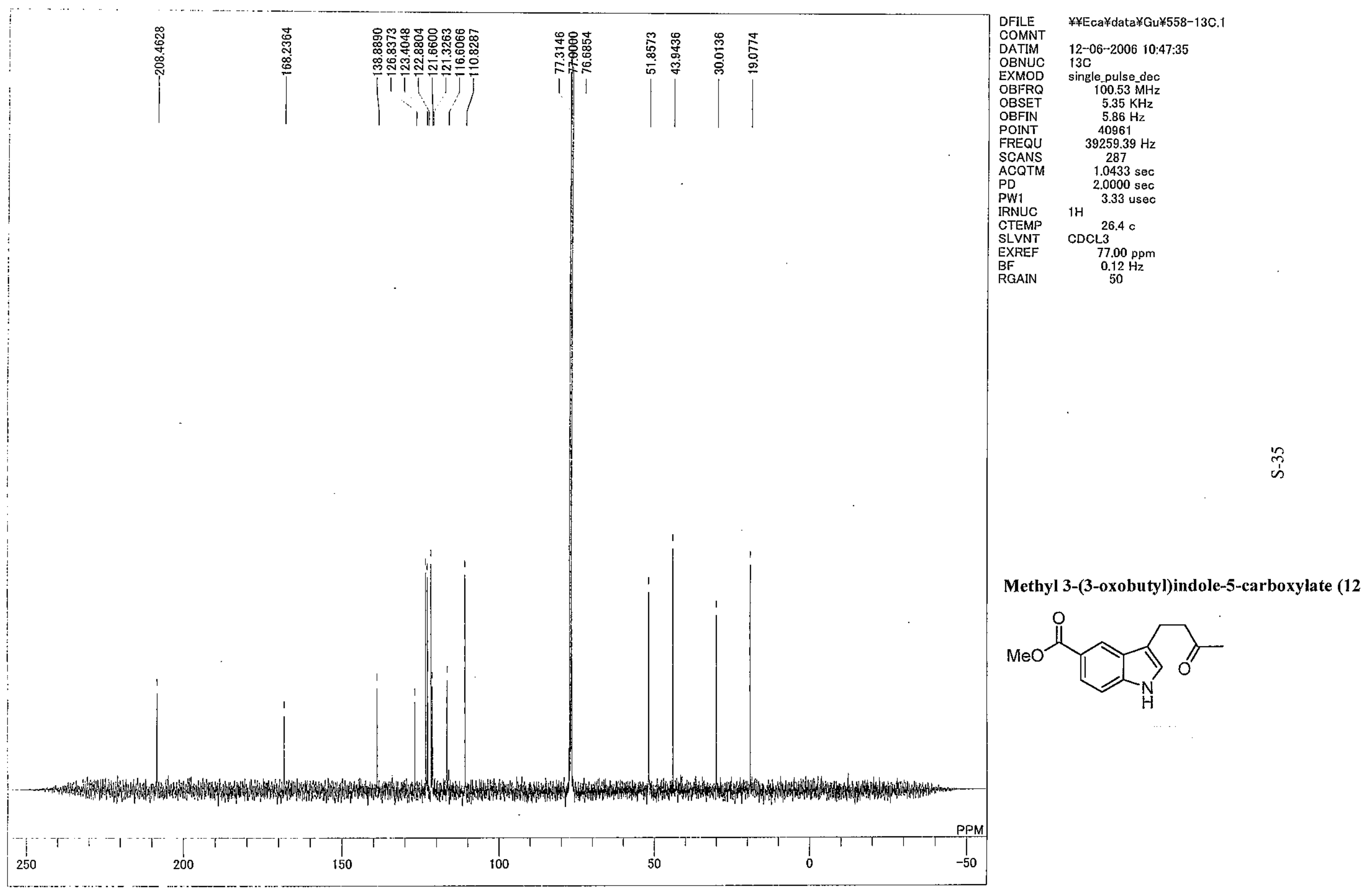




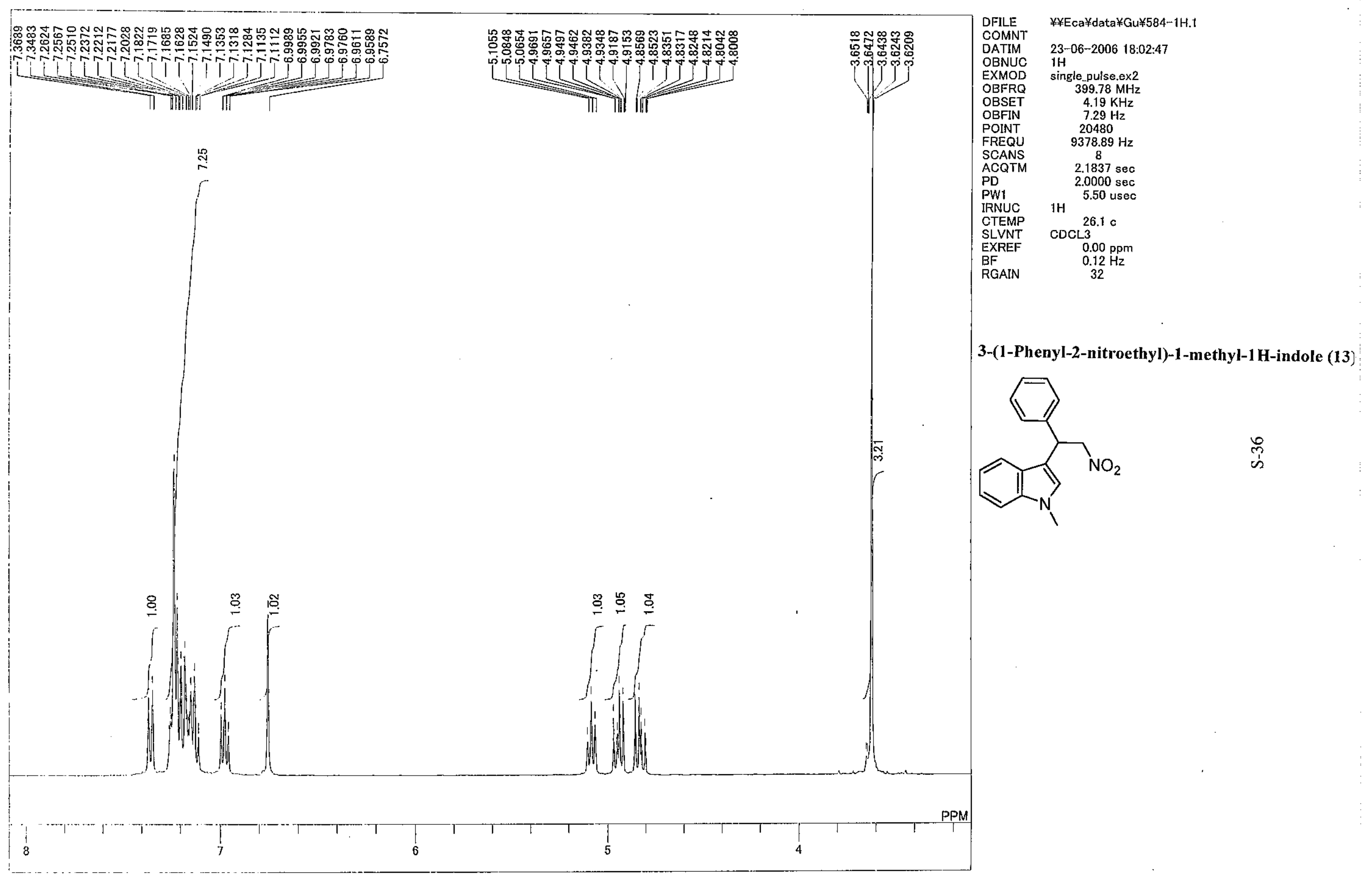




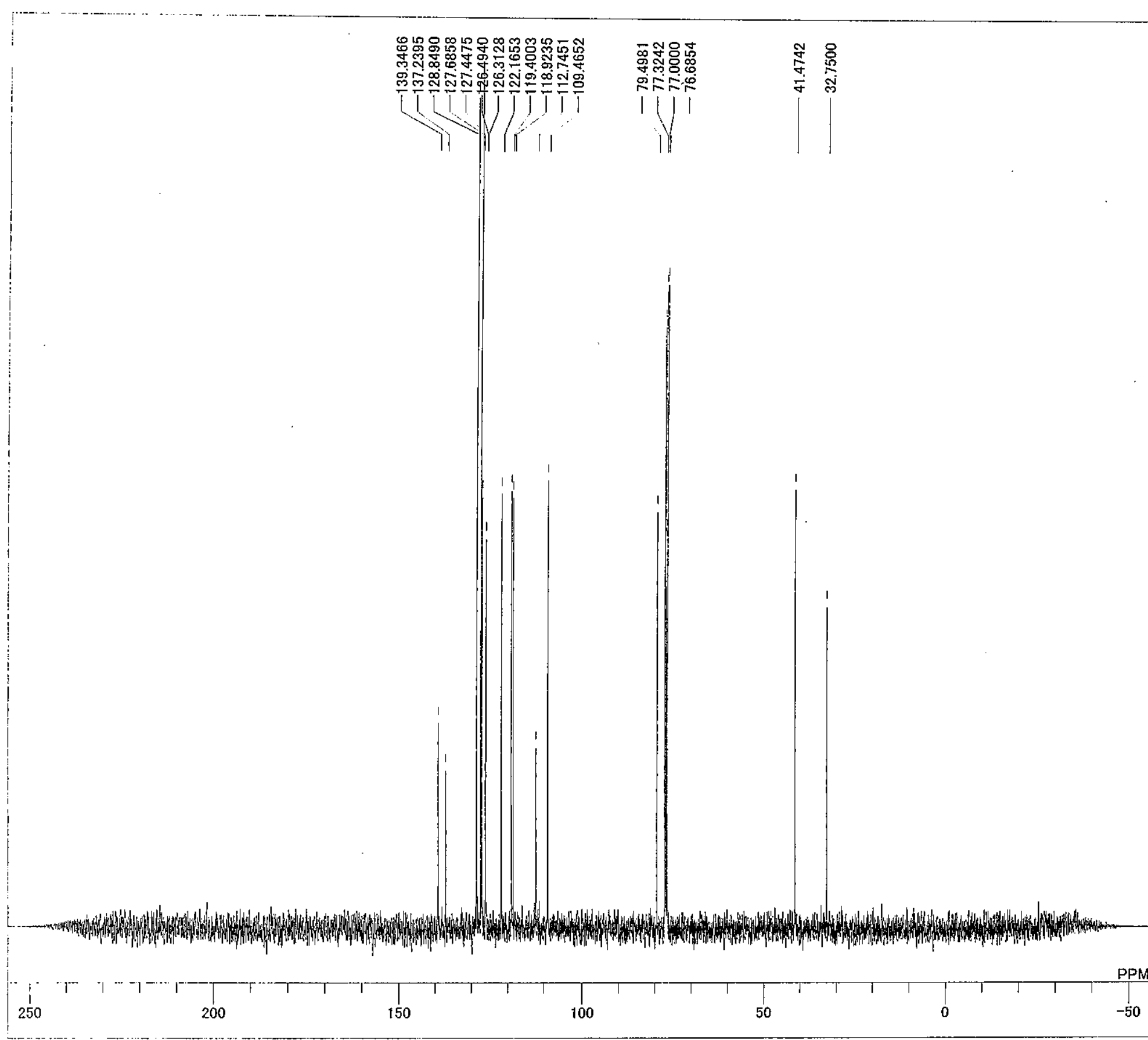

DFILE

DATIM

OBFRQ

OBSET

OBFIN

POINT

FREQU

SCANS

ACQT

PD

IRNUC

CTEMP

SLVNT

EXREF

BF
RGAIN

CDCL 3

$77.00 \mathrm{ppm}$

52

3-(1-Phenyl-2-nitroethyl)-1-methyl-1 H-indole (1:

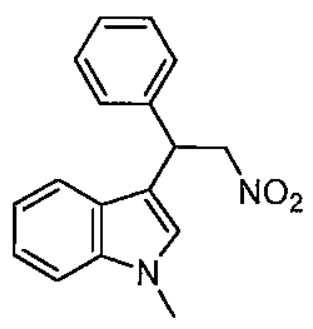


$¥ ¥$ Eca $¥$ data $¥ G u ¥ 563-1 H .1$

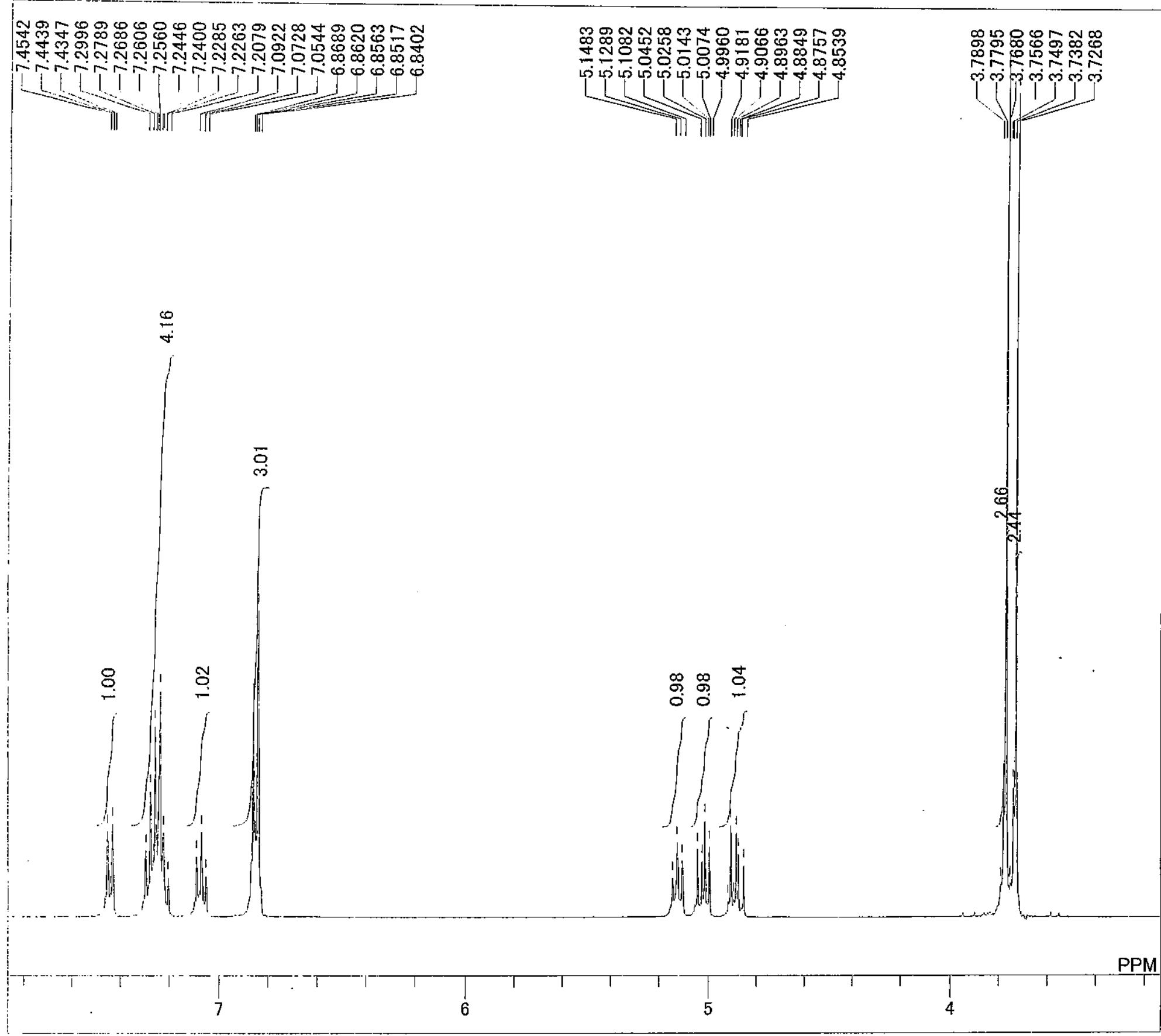

DFILE

COMNT

DATIM

EXMOD

OBFRQ

OBSET

OBFIN

POINT

FREQU

SCANS

ACQTM

ACQTN

PWN 1

CTEMP

SLVNT

EXREF

RGAIN
₹Eca $¥$ data $\because \mathrm{Gu} ¥ 563-1 \mathrm{H} .1$

16-06-2006 14:30:04

$1 \mathrm{H}$

single_pulse.ex2 $399.78 \mathrm{MHz}$ $4.19 \mathrm{KHz}$

$7.29 \mathrm{~Hz}$

$9378.89 \mathrm{~Hz}$

$2.1837 \mathrm{sec}$ $2.0000 \mathrm{sec}$

5.50 usec

(

$\mathrm{CDCL}^{2} 3$

$7.24 \mathrm{ppm}$

$7.24 \mathrm{ppm}$

$0.12 \mathrm{~Hz}$

32

3-[1-(4-Methoyphenyl)-2-nitroethyl]1-methyl- $\boldsymbol{H}$-indole (14)

$\mathrm{MeO}$

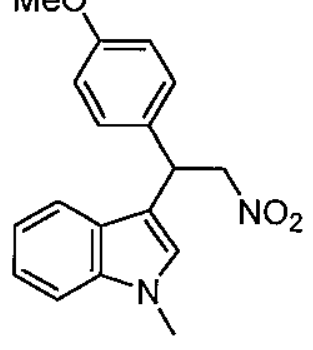




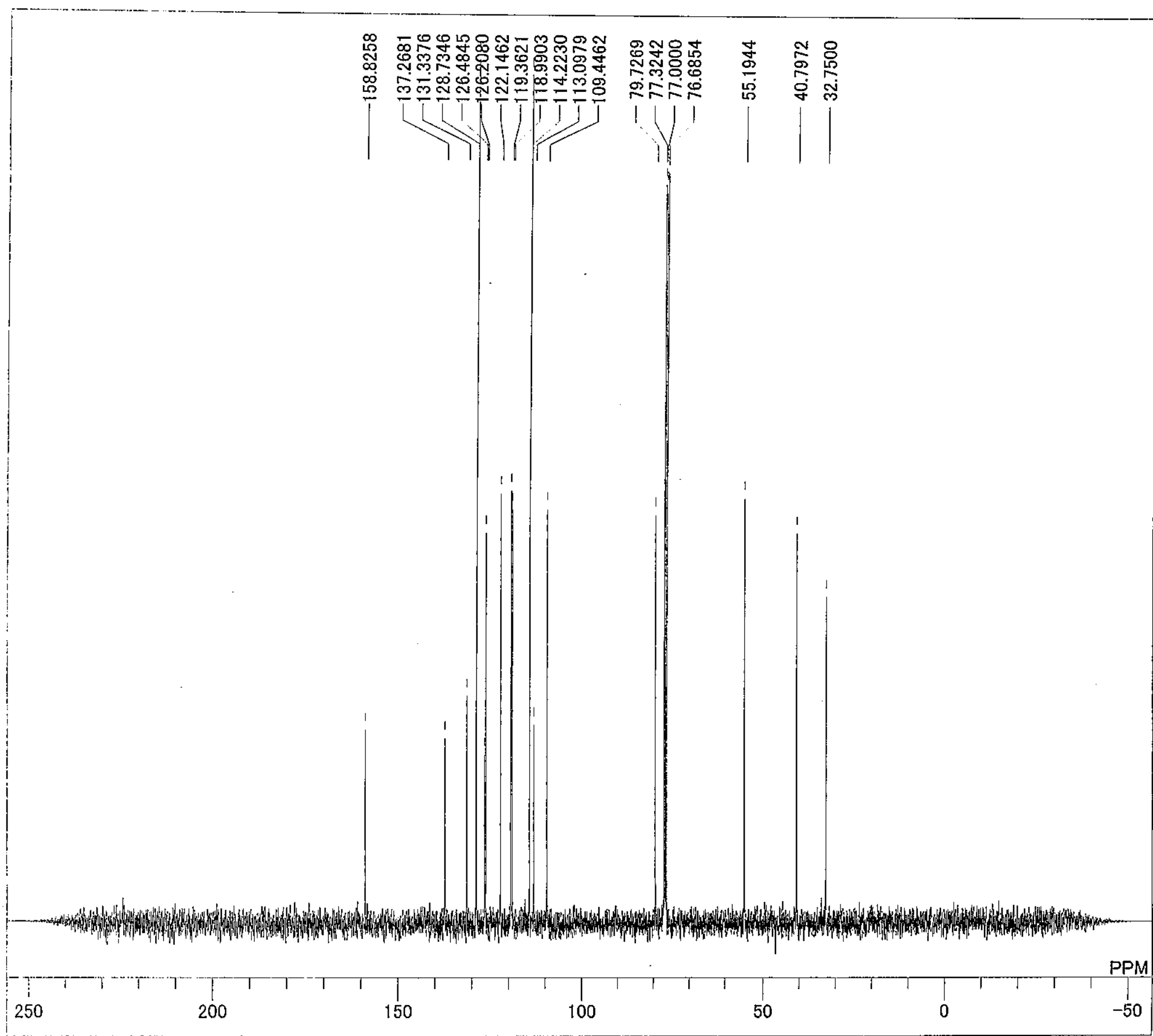

DFILE

COMNT

DATIM

EXMOD

OBSET

OBFIN

PRE

SCANS

ACQTM

PD

IRNUC

CTEMP

SLVNT

EXREF

EXREF

RGAIN

$1 \mathrm{H}$

$\operatorname{CDCL} 3$

$77.00 \mathrm{ppm}$

$0.12 \mathrm{~Hz}$

$\stackrel{m}{\dot{m}}$

3-[1-(4-Methoyphenyl)-2-nitroethyl]1-methyl- $1 H$-indole (14)

$\mathrm{MeO}$

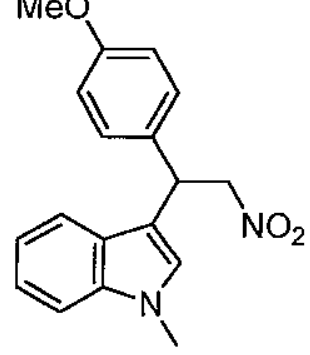




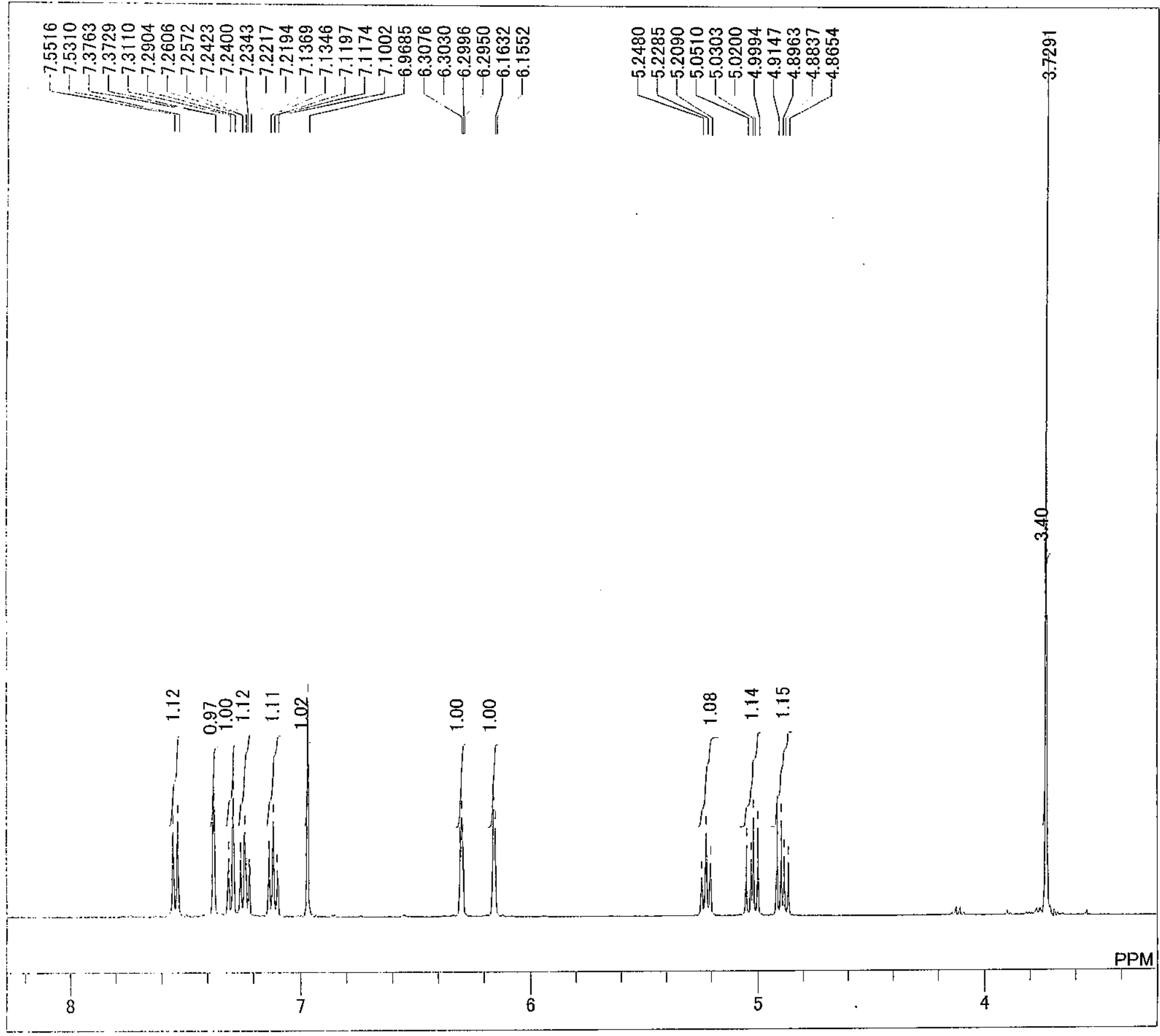

DFILE

COMNT

DATIM

OBNUC

EXMOD

OBFRQ

OBSET

OBFIN

PREQU

SCANS

ACQTM

PD

PW1

IRNUC

CTEMP

SLVNT

EXREF

BF

RGAIN

$¥ ¥ E \mathrm{ca} ¥$ data $¥ \mathrm{Gu} ¥ 559-1 \mathrm{H} .1$

14-06-2006 09:27:30

iH

single_pulse.ex2 399.78 $\mathrm{MHz}$ $4.19 \mathrm{KHz}$ $7.29 \mathrm{~Hz}$ 20480 $9378.89 \mathrm{~Hz}$ $2.1837 \mathrm{sec}$ $2.0000 \mathrm{sec}$

$1 \mathrm{H}$ 5.50 usec

25.8 CDCL.3 $7.24 \mathrm{ppm}$ $0.12 \mathrm{~Hz}$
32

3-(1-Furan-2-yl-2-nitroethyl)1-methyl-1 $H$-indole (15)

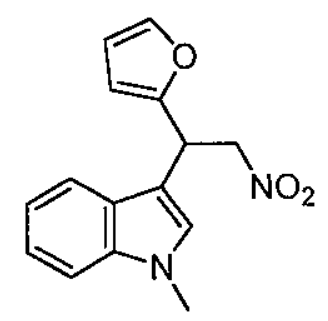


$¥ ¥ \mathbb{E}$ ca $¥$ data $¥ G u ¥ 559-13 \mathrm{C} .1$

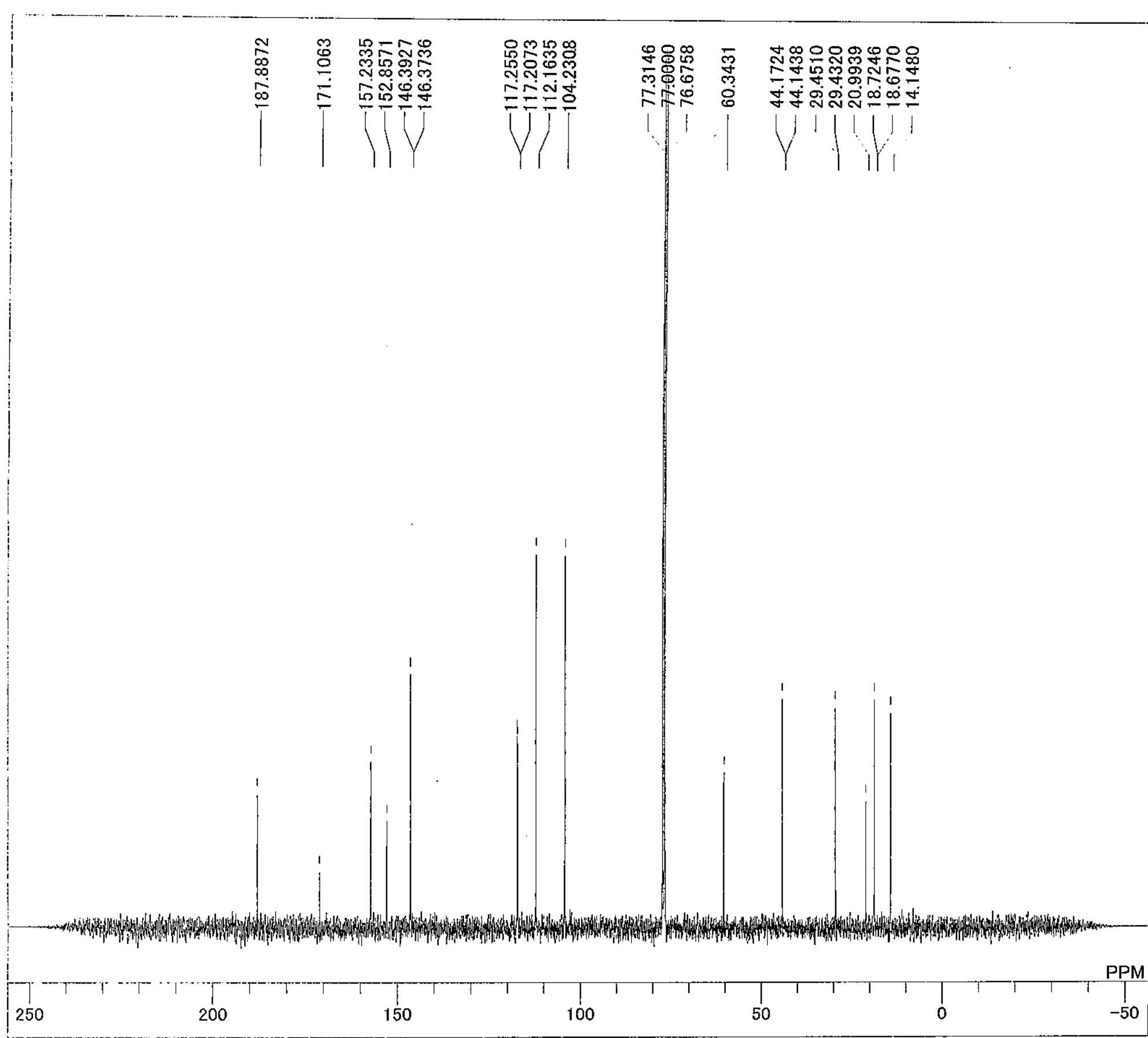

DFILE

DATIM

OBNUC

OBFRQ

OBSET

OBFIN

FREQU

SCANS

ACQTM

PD

PW1

IRNUC

CTEMP

SLVNT

EXREF

BF

RGAIN

3-06-2006 14:08:21

$13 \mathrm{C}$

single_pulse_dec

$00.53 \mathrm{MHz}$

$5.35 \mathrm{KHz}$

$5.86 \mathrm{~Hz}$

40961

$39259.39 \mathrm{~Hz}$

$1.0433 \mathrm{sec}$

$2.0000 \mathrm{sec}$

$1 \mathrm{H}$

usec

CDCL3

$77.00 \mathrm{ppm}$

$0.12 \mathrm{~Hz}$

56

3-(1-Furan-2-yl-2-nitroethyl)1-methyl- $1 H$-indole (15)

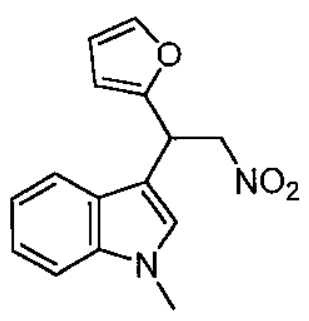




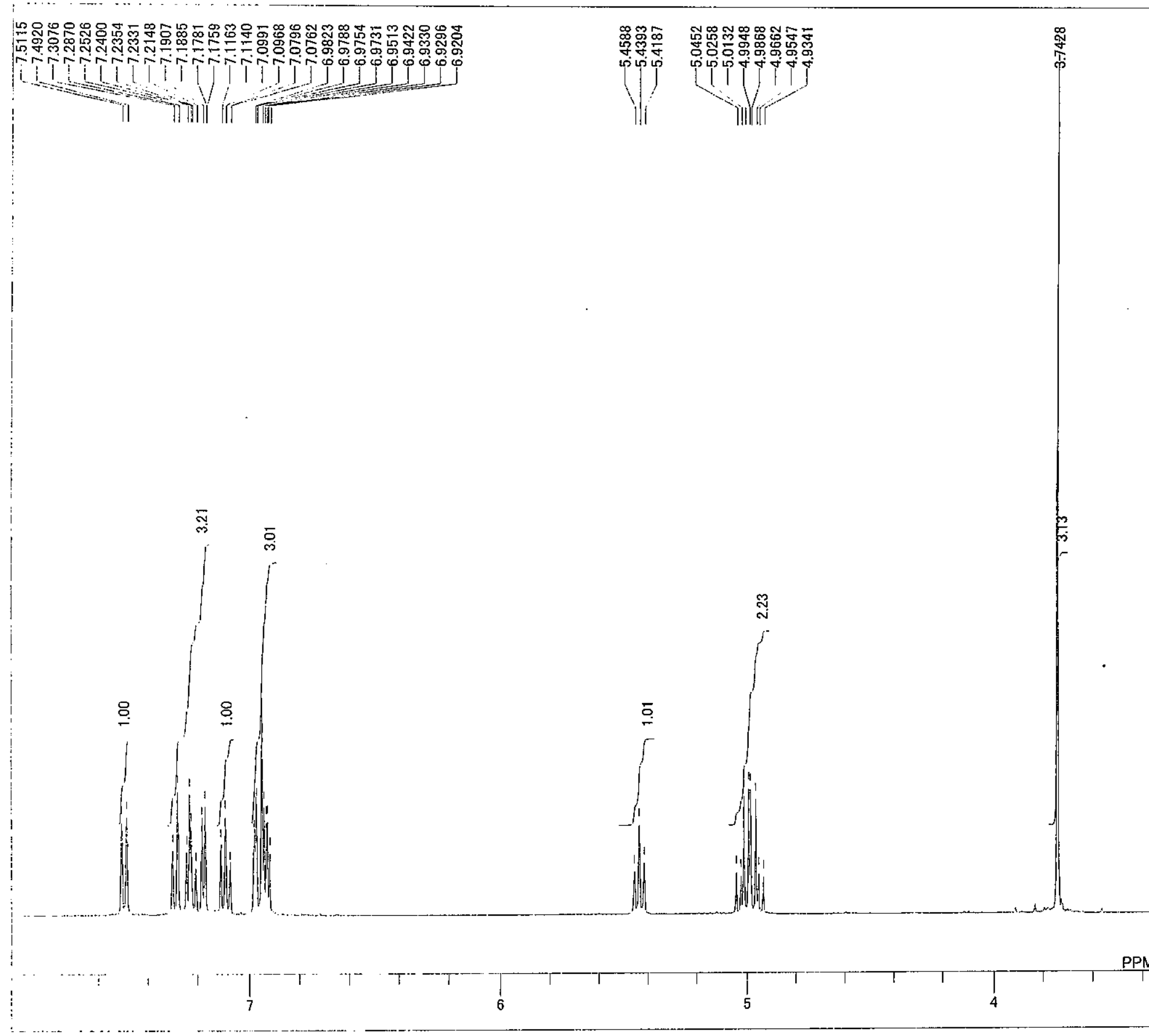

DFILE

DATIM

EXMOD

OBFRQ
OBSET

OBSET

POINT

FREQU

SCANS

ACQTM

PD

IRNUC

CTEMP

SLVNT

EXRE

RGAIN

14-06-2006 09:37:02

se.ex2

(3.78 $\mathrm{MHz}$

$4.19 \mathrm{KHz}$

$7.29 \mathrm{~Hz}$

$9378.89 \mathrm{~Hz}$

8

$2.1837 \mathrm{sec}$

$5.50 \mathrm{sec}$

$1 \mathrm{H}$

$\mathrm{CDCL3}$

$7.24 \mathrm{ppm}$
$0.12 \mathrm{~Hz}$

$0.12 \mathrm{~Hz}$

1-Methyl-3-(2-nitro-1-thiophen-2ylethyl)-1 $\mathrm{H}$-indole (16)

$\underset{y}{y}$

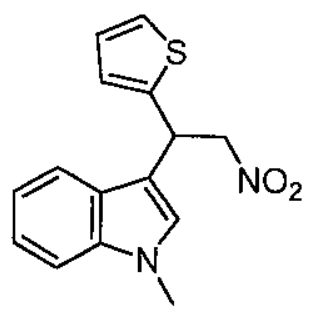




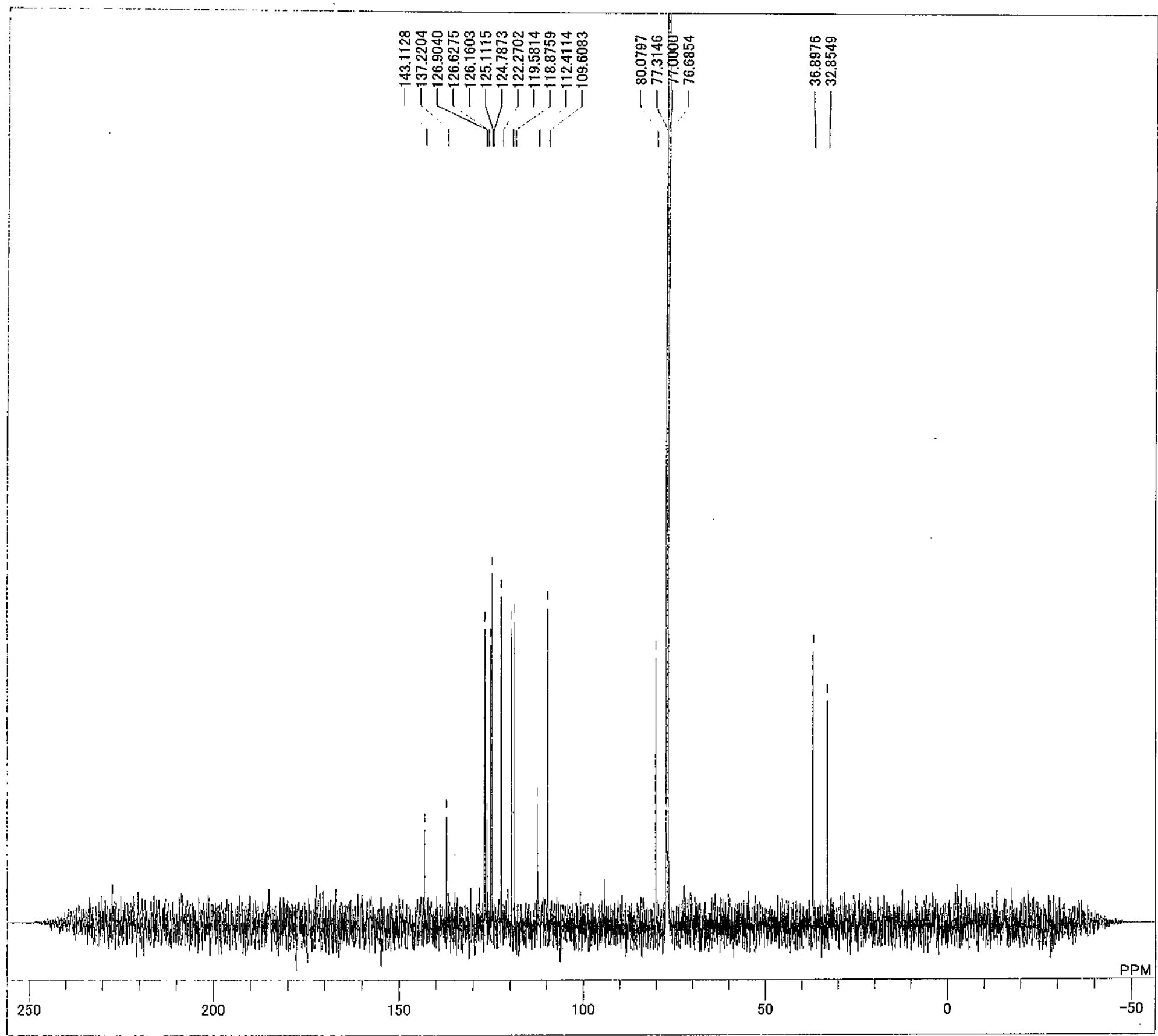

DFILE

COMNT

EXMOD

OBFRQ

OBSET

OBFIN

POINT

FREQU

SCANS

ACQTM

ACQTM

PNUC

CTEMP

SLVNT

EXREF

RGAIN

$\mathrm{CDCL}$

77.00

$0.12 \mathrm{~Hz}$
50

1-Methyl-3-(2-nitro-1-thiophen-2ylethyl)-1H-indole (16)

$\stackrel{m}{i}$

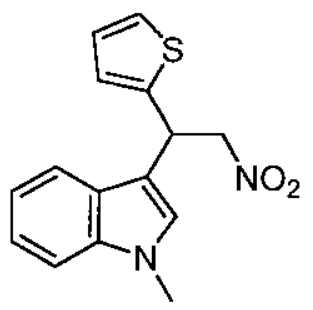




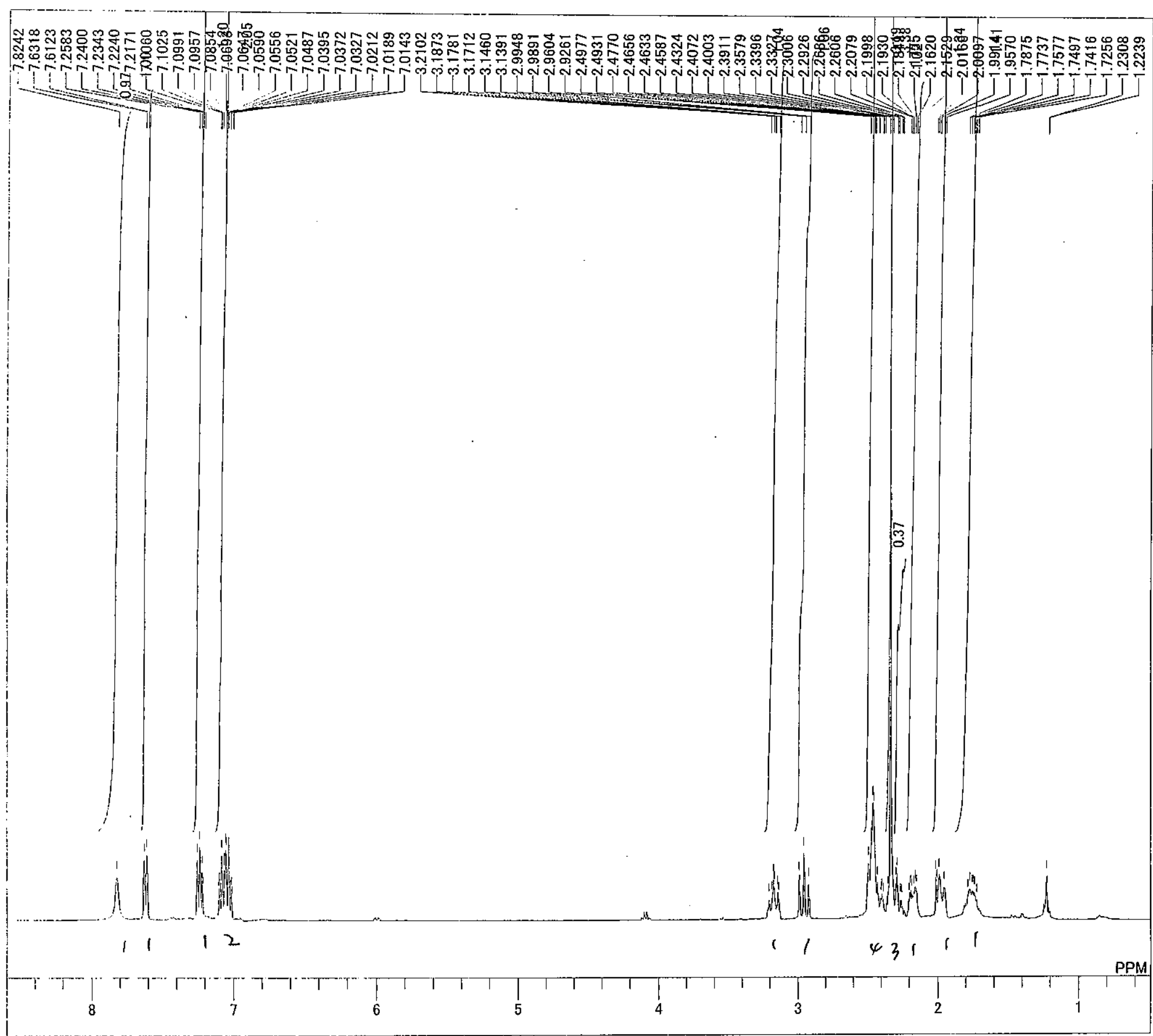

DFILE

DATIM

OBNUC

EXMOD

OBFRQ

OBSE

OBFIN

POINT

FCANS

ACATM

PW1

IRNUC

SLVNT

EXREF

BF

CDCL

$\operatorname{CDCL}^{25.8}$

$7.24 \mathrm{ppm}$

32

3-(2-Methyindol-3-yl)cyclohexanone (17)

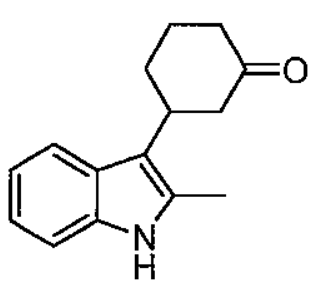

过 
$¥ ¥ \mathrm{Eca} ¥ \mathrm{data} ¥ \mathrm{Gu} ¥ 580-\mathrm{m}-13 \mathrm{C} .1$

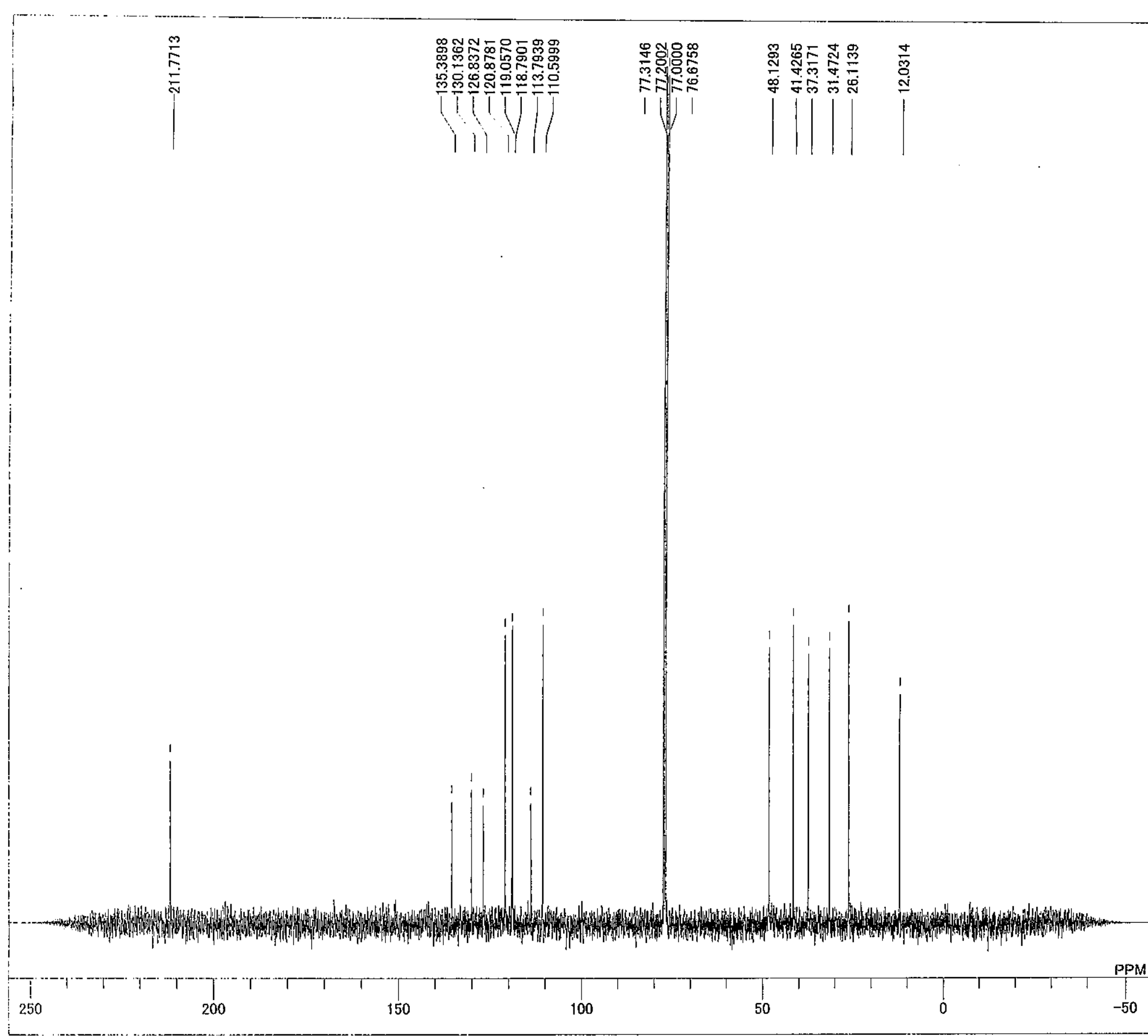

DFILE

COMNT

EXMOD

OBFRQ

OBSET

OBFIN

POINT

FREQU

SCANS

ACQT

PD

IRNUC

CTEMP

SLVNT

EXREF

BF
RGAIN

CDCL 3

$77.00 \mathrm{ppm}$

56

3-(2-Methyindol-3-yI)cyclohexanone (17)

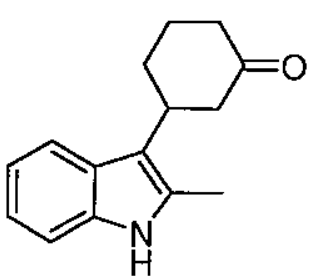




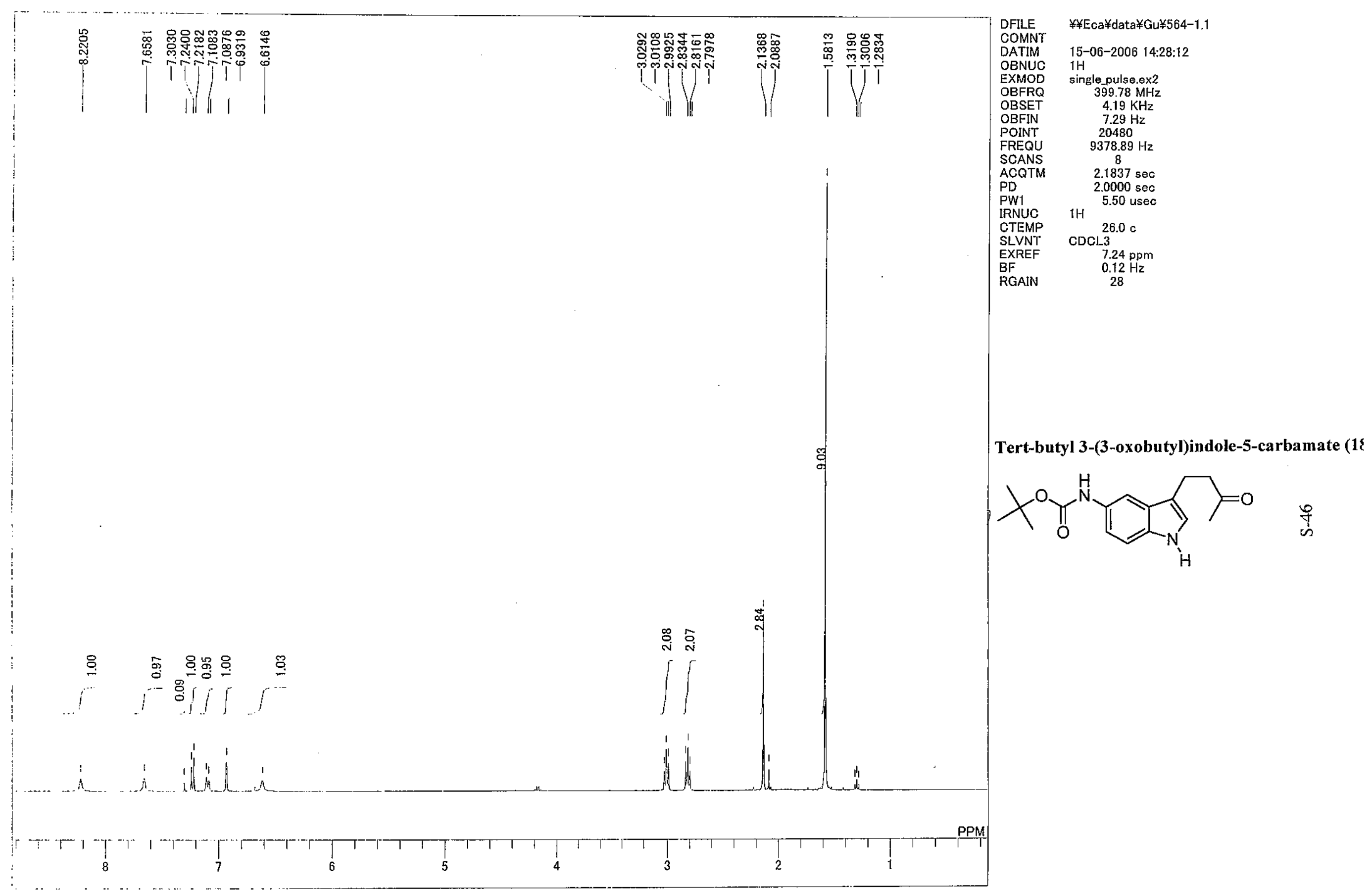


¥¥Eca $¥$ data $¥ G u ¥ 564-1-13 C .1$

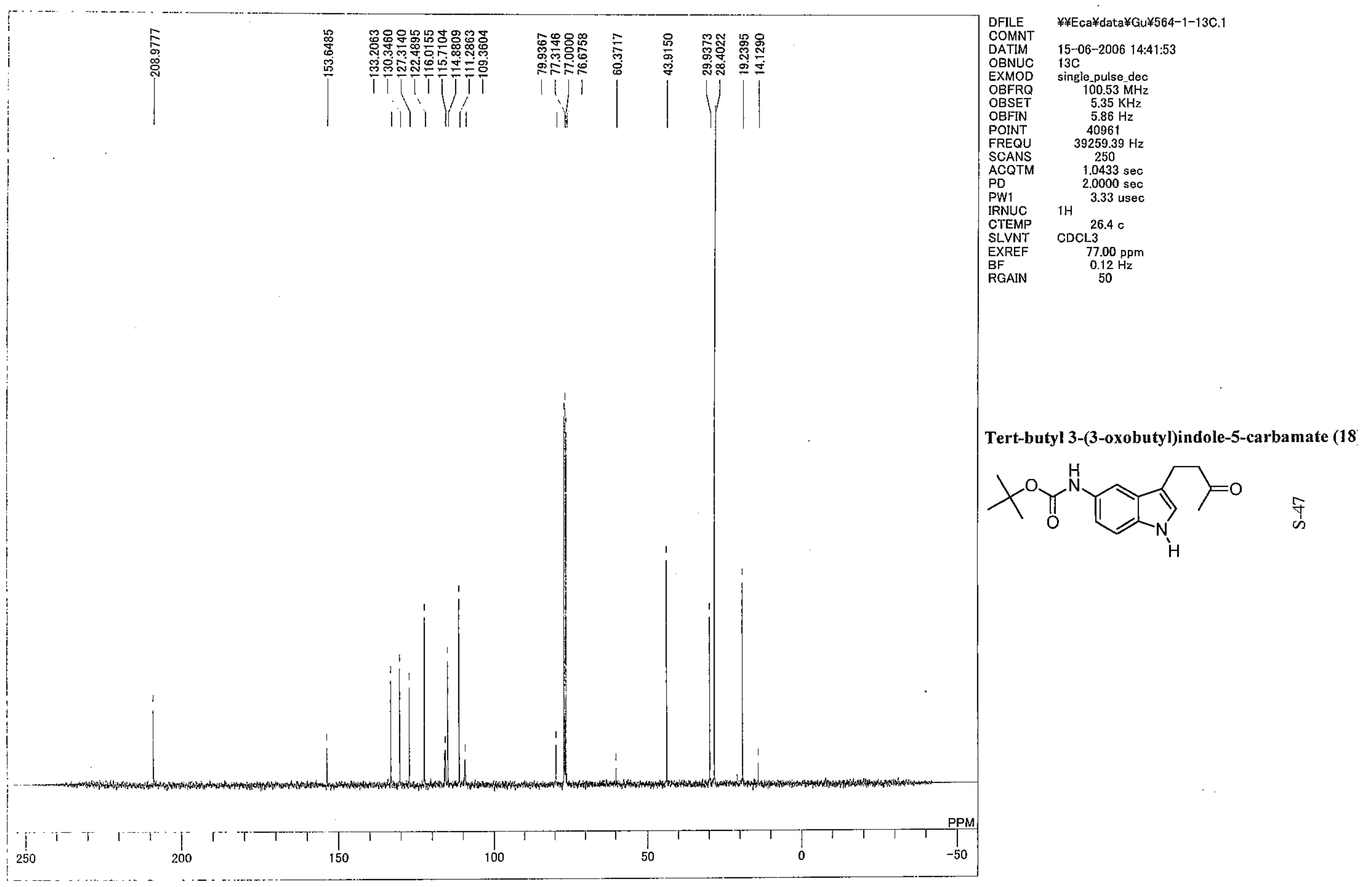


$¥ ¥$ Eca $¥$ data $¥ G u ¥ 574.1$

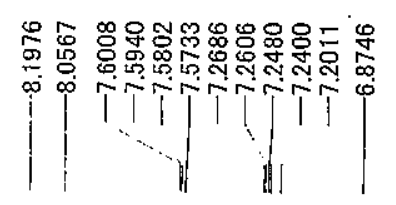

EXMOD

single_pulse.ex2

$399.78 \mathrm{MHz}$ $4.19 \mathrm{KHz}$ $7.29 \mathrm{~Hz}$ 20480

POINT

FREQU

ACOTM

AD

PW1

$2.1837 \mathrm{sec}$

IRNUC

CTEMP

SLVNT

BF

$2.0000 \mathrm{sec}$
$5.50 \mathrm{usec}$

25.7 c

$7.24 \mathrm{ppm}$

$0.12 \mathrm{~Hz}$

4-(5-(4,4,5,5-Tetramethyl-1,3,2-

dioxaborolan-2-yl)-3-indolyl)-2-butanone (19)

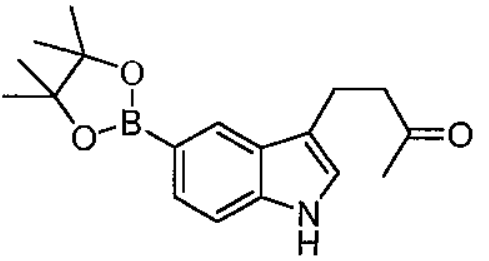

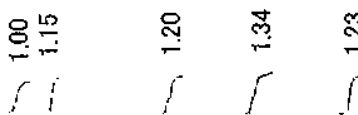

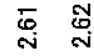

$\Gamma$

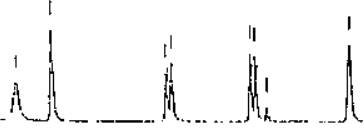

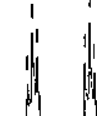

㟧

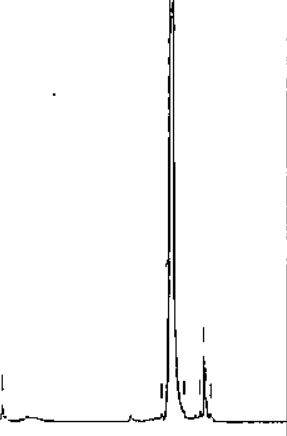

PPM

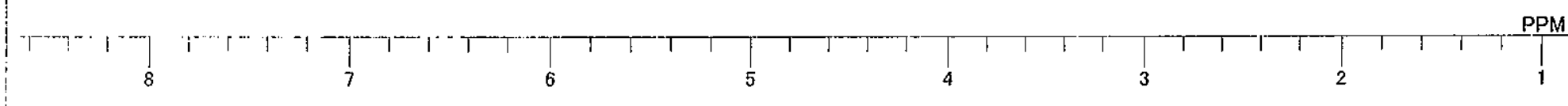




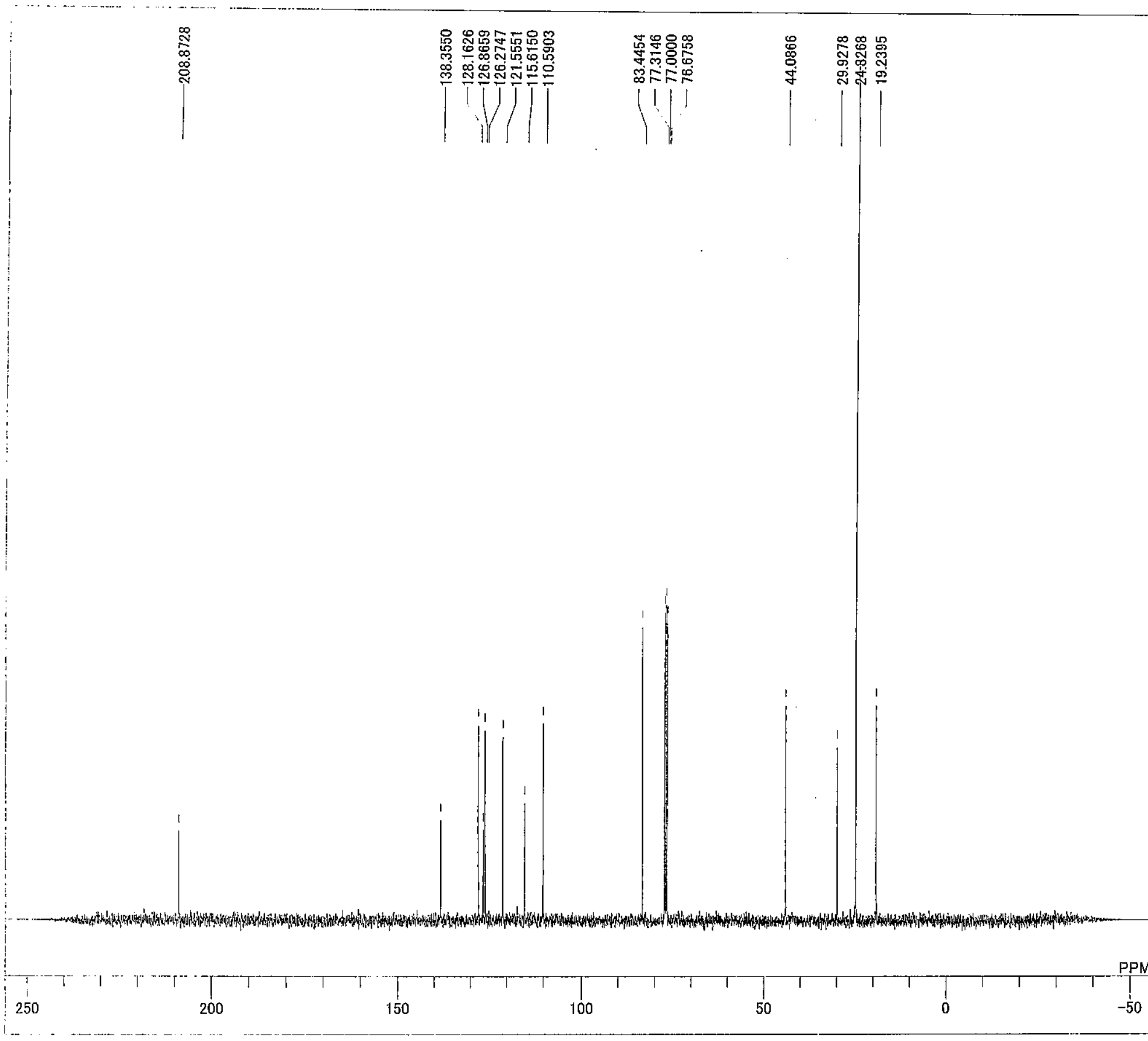

DFILE

DATIM

OBNUC

EXMOD

OBFRQ

OBSET

POINT

POINT

FREQU

SCANS

PD

PW1

IRNUC

CTEMP

SLVNT

RGAIN

CDCL3

$.00 \mathrm{ppm}$

$0.12 \mathrm{~Hz}$

$13 \mathrm{C}$

gle_pulse_dec

$5.35 \mathrm{KHz}$

$5.86 \mathrm{~Hz}$

4051

73
1.0433

$2.0000 \mathrm{sec}$

3.33 usec

54

4-(5-(4,4,5,5-Tetramethyl-1,3,2-

dioxaborolan-2-yl)-3-indolyl)-2-butanone (19)

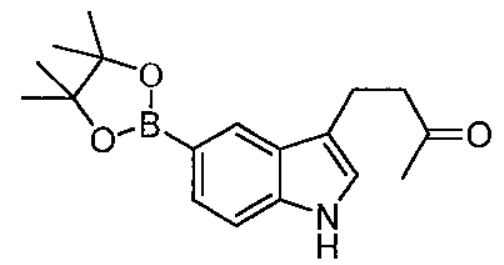




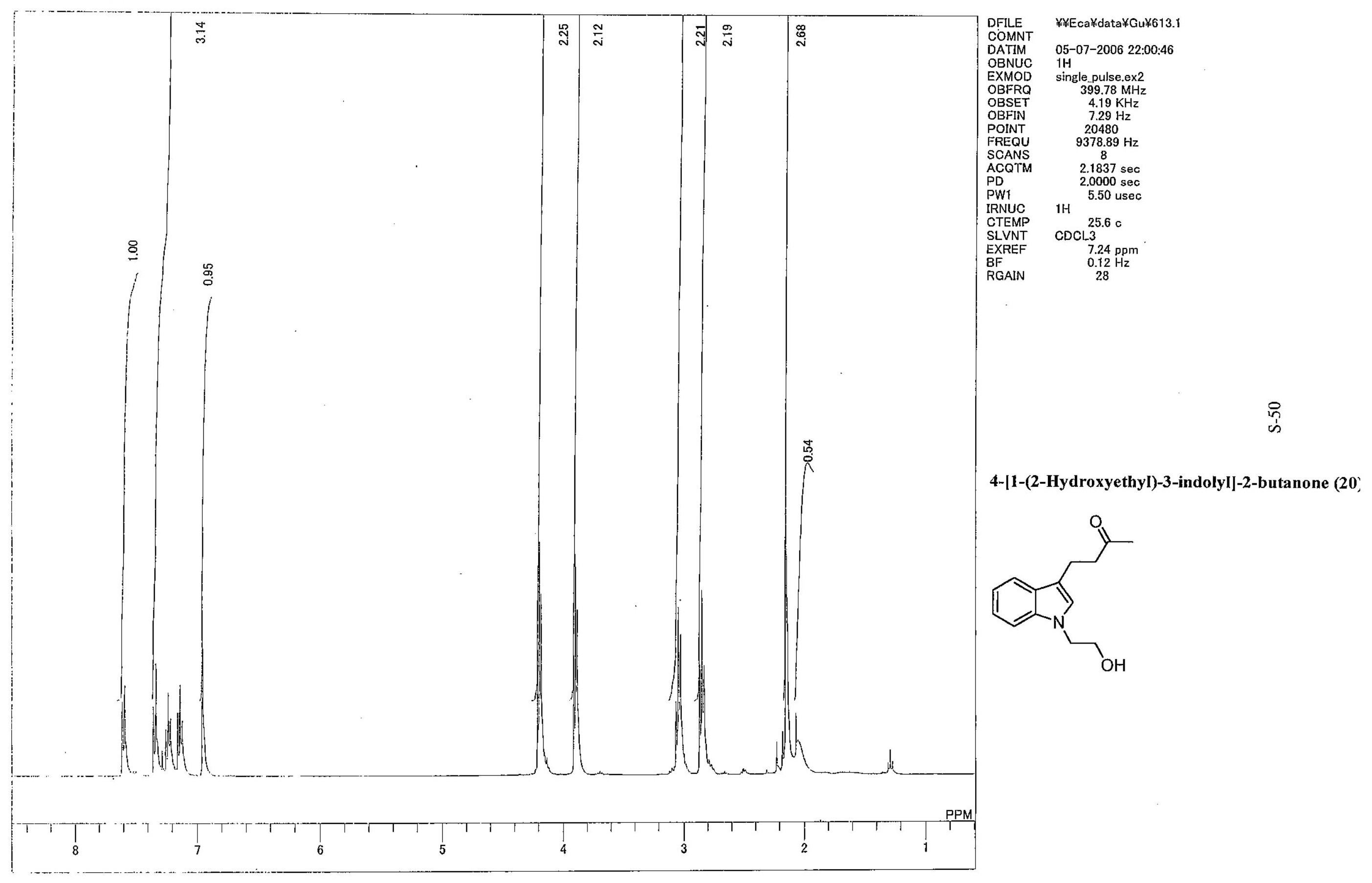




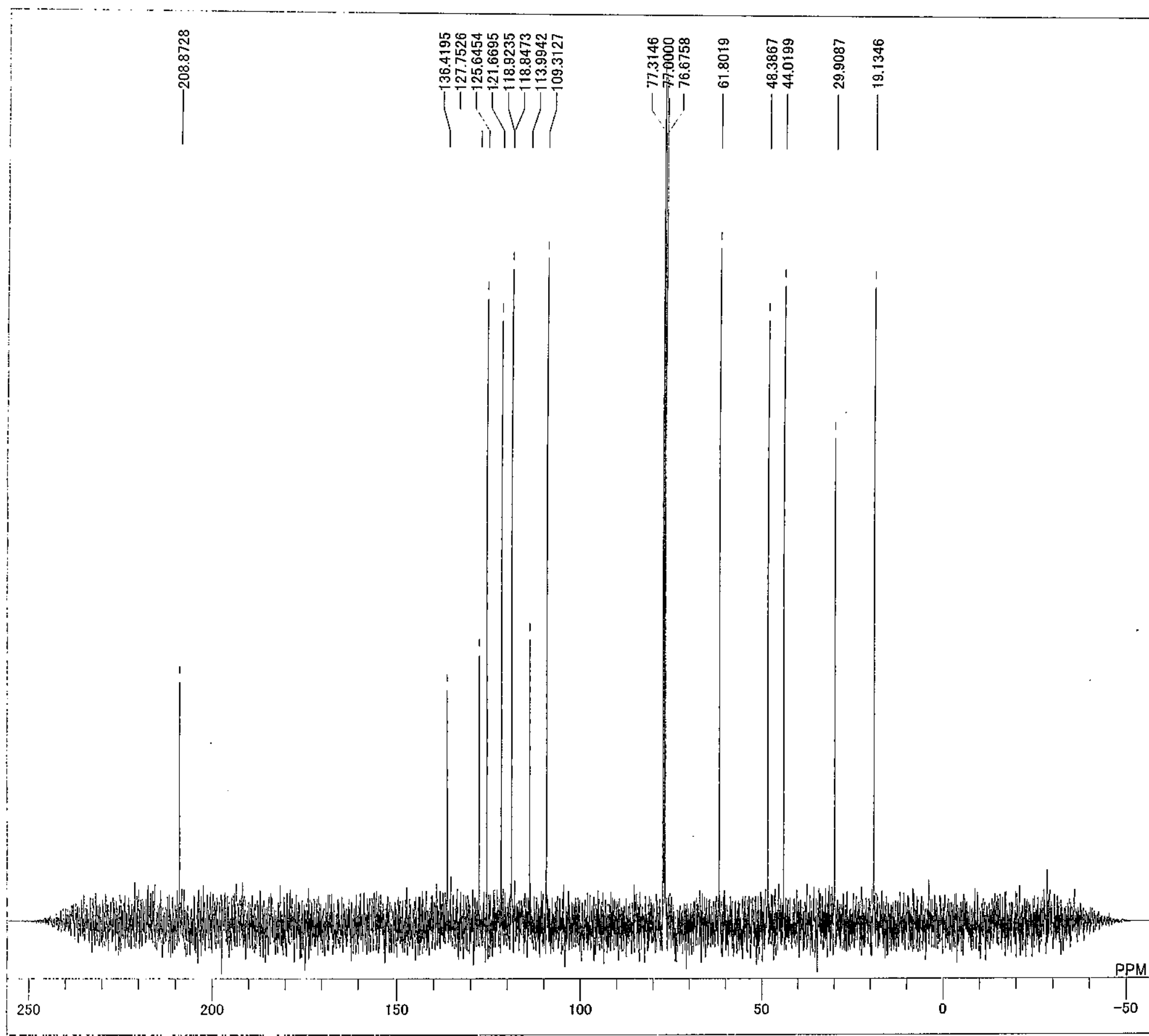

DFILE

DATIM

EXMOD

OBFRQ

OBSE

OBFIN

POINT

SCANS

ACQTM

ACQ

CTEMP

SLVNT

EXREF

RGAIN
¥¥Eca $¥$ data $¥ G u ¥ 613-13 \mathrm{C} .1$

05-07-2006 22:07:06

single_pulse_dec

$100.53 \mathrm{MHz}$
$5.35 \mathrm{KHz}$

$5.86 \mathrm{~Hz}$

40961

$39259.39 \mathrm{~Hz}$

$1.0433 \mathrm{sec}$

$2.0000 \mathrm{sec}$

$2.000 \mathrm{sec}$
usec

26.10

$\mathrm{COCL} 3$

$77.00 \mathrm{ppm}$
$0.12 \mathrm{~Hz}$
54

4-[1-(2-Hydroxyethyl)-3-indolyl]-2-butanone (21

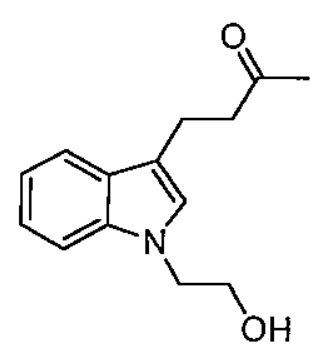


¥¥Eca¥data $¥ G u ¥ 615.1$

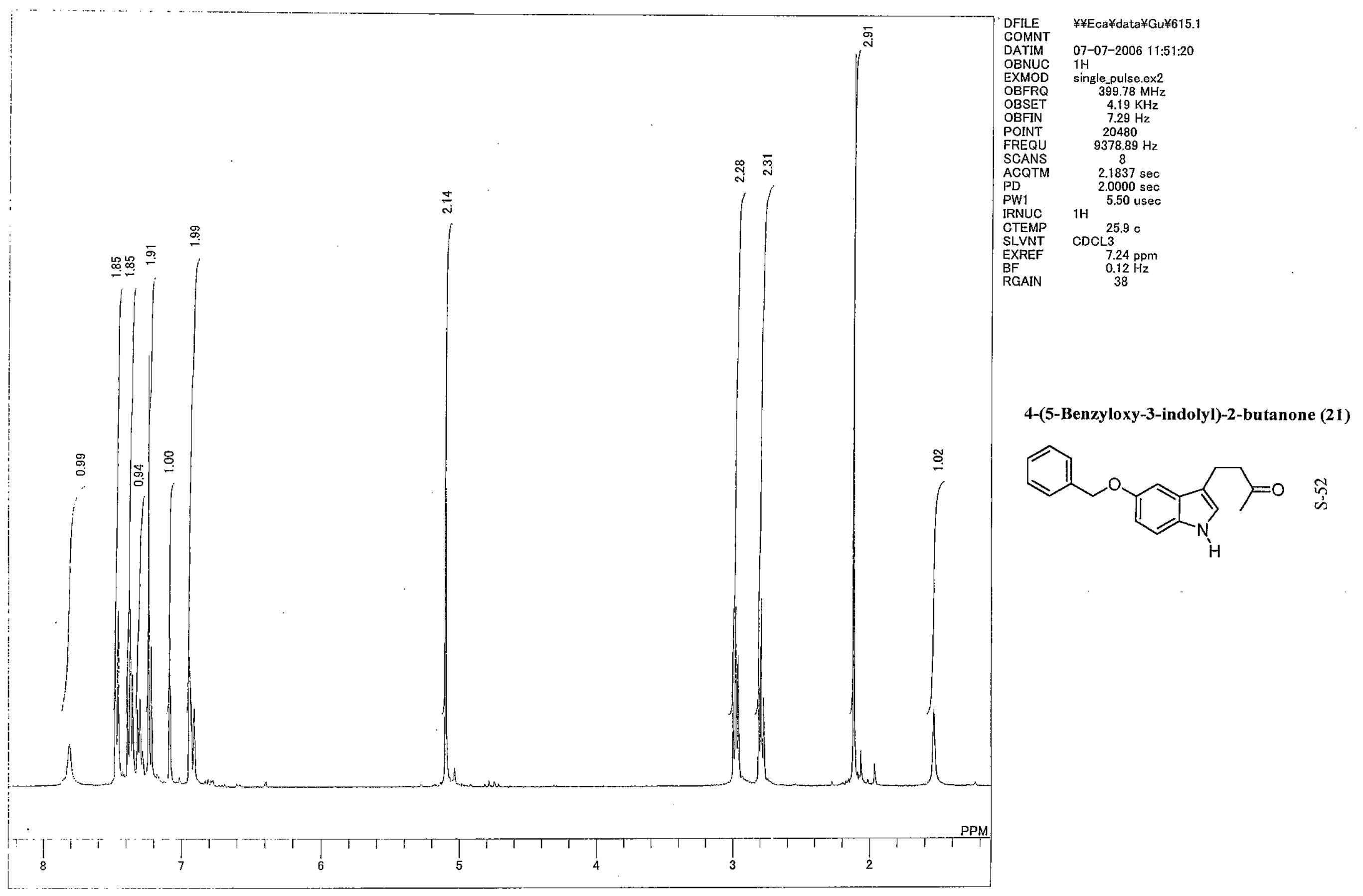




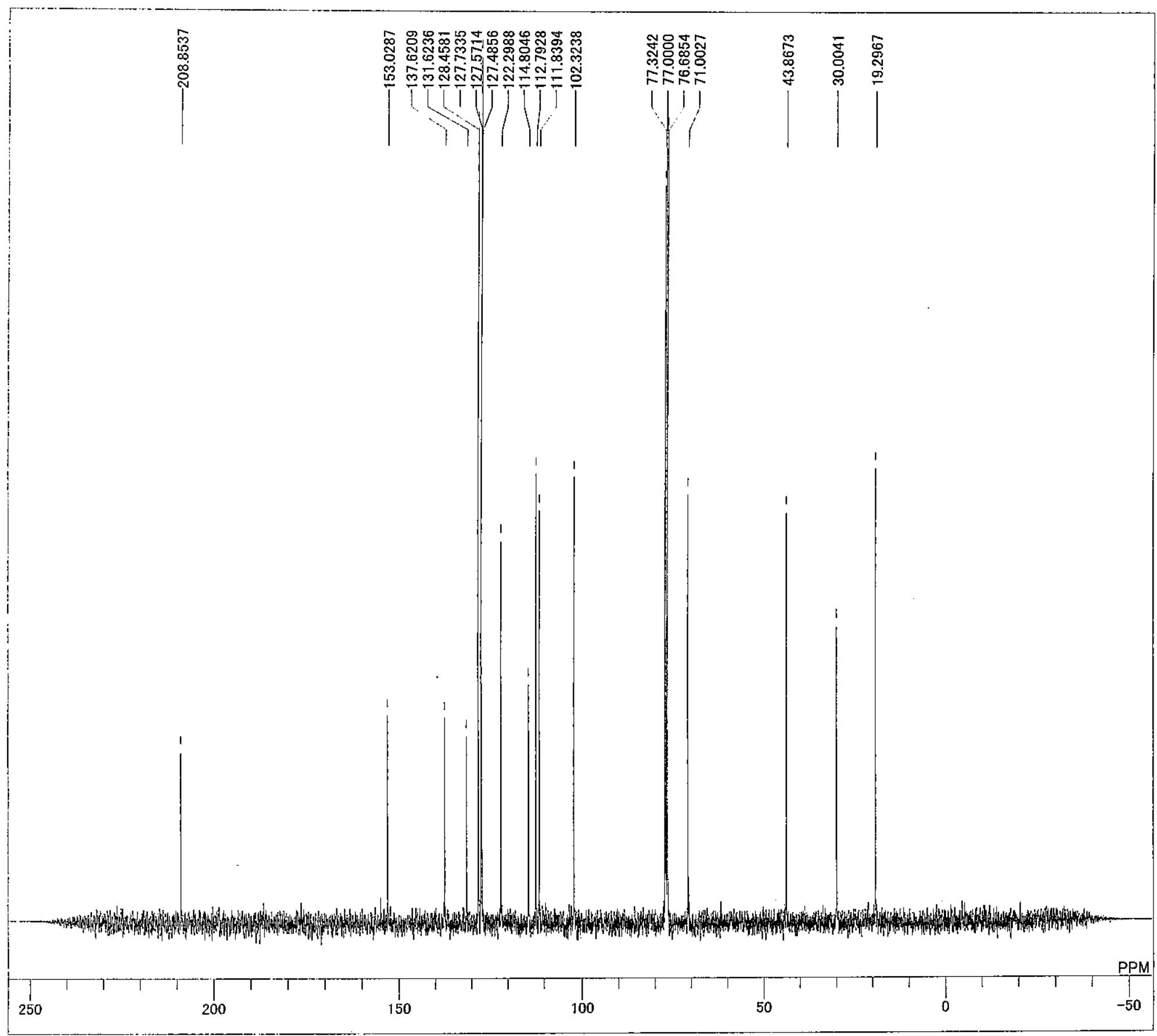

DFILE

COMNT

DATIM

OBNUC

EXMOD

OBFRQ

OBSET

PBINT

FREQU

SREQU

ACQTM

PD

PW1

IRNUC

CTEMP

SLVNT

BF

RGAIN

FEca $\approx$ data $¥ G u * 616-13 C .1$

07-07-2006 14:11:07

ingle.pulse dec
$100.53 \mathrm{MHz}$ $5.35 \mathrm{KHz}$ $5.86 \mathrm{~Hz}$

$39259.39 \mathrm{~Hz}$

3925.39
187

$1.0433 \mathrm{sec}$

$2.0000 \mathrm{sec}$

26.0

$77.00 \mathrm{ppm}$

$0.12 \mathrm{~Hz}$

4-(5-Benzyloxy-3-indolyl)-2-butanone (21)

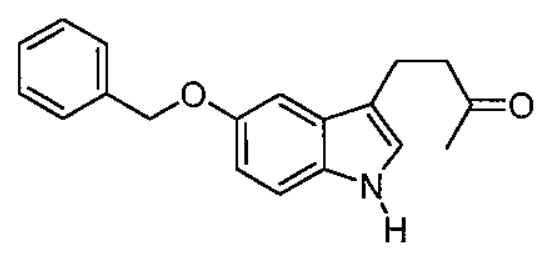

$\stackrel{m}{n}$ 
¥¥Eca data $¥ \mathrm{G} u \neq 678.1$

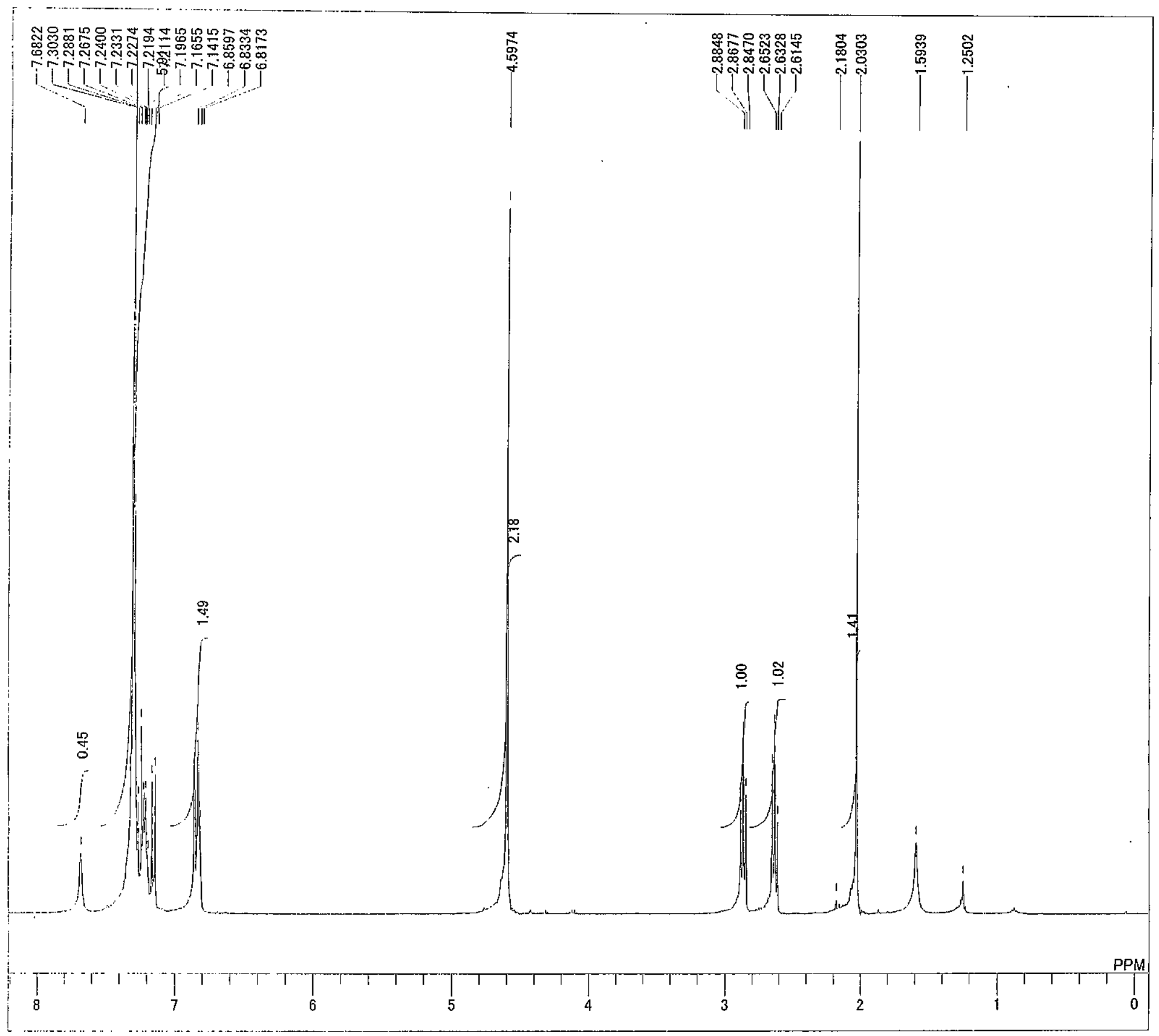

DFILE

DATIM

OBNUC

EXMOD

OBFRQ

OBFIN

POINT

PREOI

FREQL

ACQTM

$\mathrm{PD}$

PW1 1

IRNUC

SLVNT

EXREF

BF

4-(5-Dibenzylamino-1 $H$-indol-3-yl) butan-2-one (22)

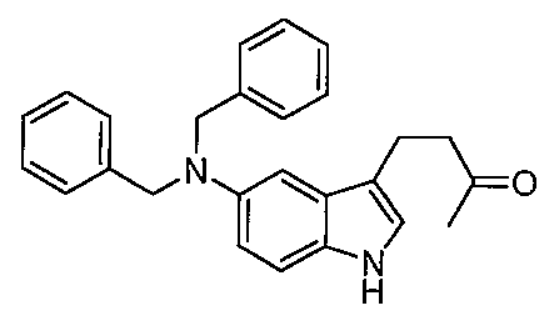




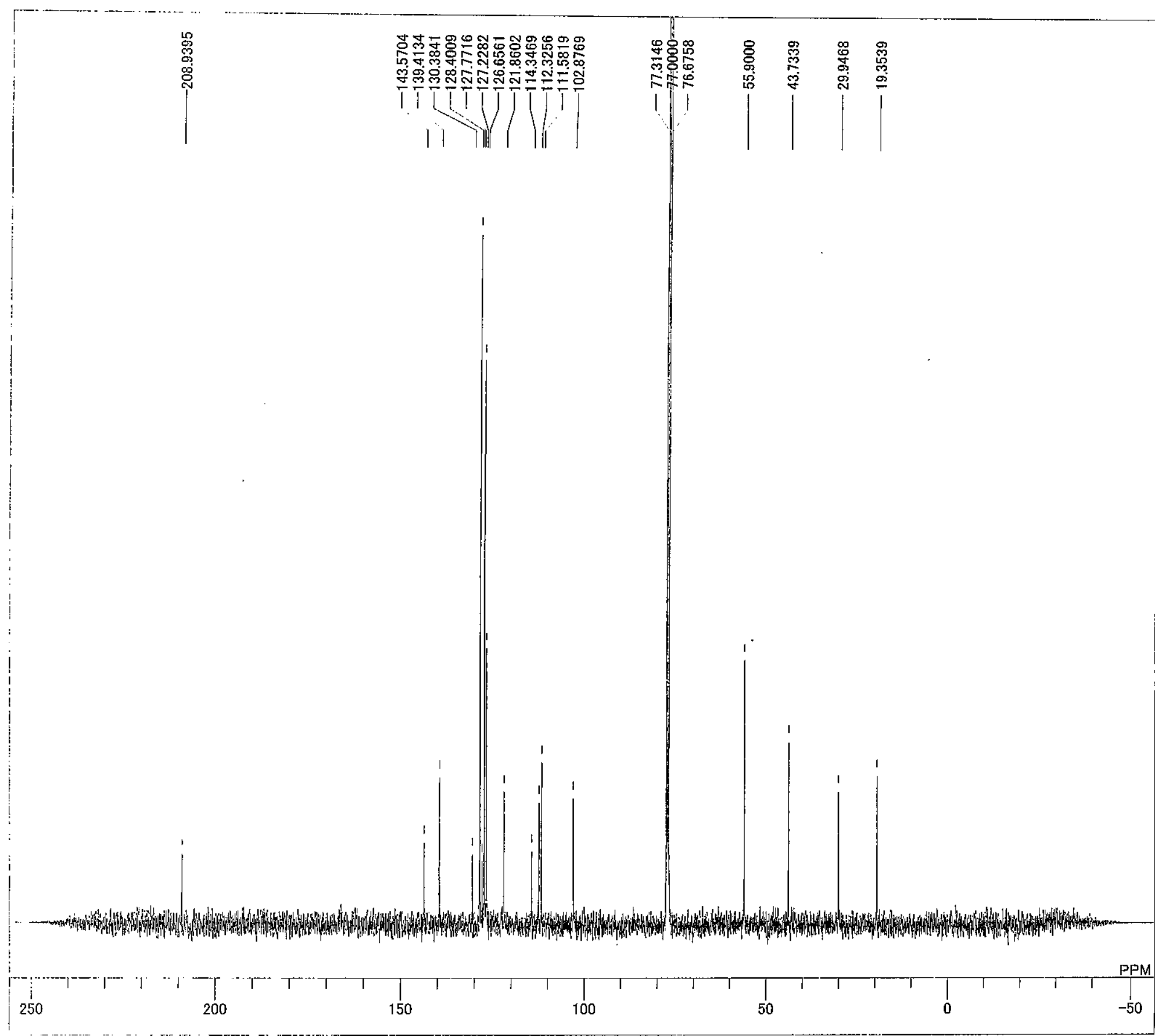

DFILE

COMNT

DATIM

EXMOD

EXMOD

OBFRO

OBFIN

POINT

FREQL

SCANS

ACQTM

PD

IRN1

CTEMP

SLVNT

EXREF

BF

RGAIN

¥¥Eca $¥$ data $¥ G u \neq 678-13 \mathrm{C} .1$

2-08-2006 17:13:25

ingle_pulse_dec

$100.53 \mathrm{MHz}$

$5.86 \mathrm{~Hz}$

40961

$39259.39 \mathrm{~Hz}$

327

$2.0000 \mathrm{sec}$

3.33 usec

$24.2 \mathrm{c}$

$77.00 \mathrm{ppm}$

$0.12 \mathrm{~Hz}$

4-(5-Dibenzylamino- $1 H$-indol-3-yl)-

butan-2-one (22)

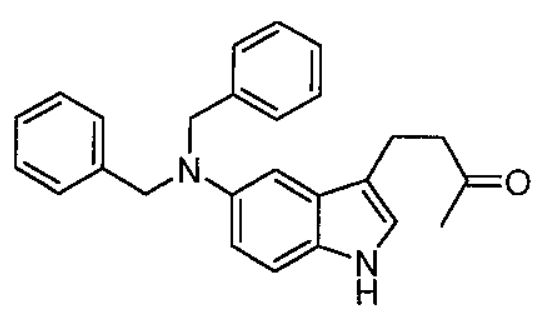




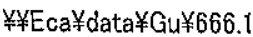

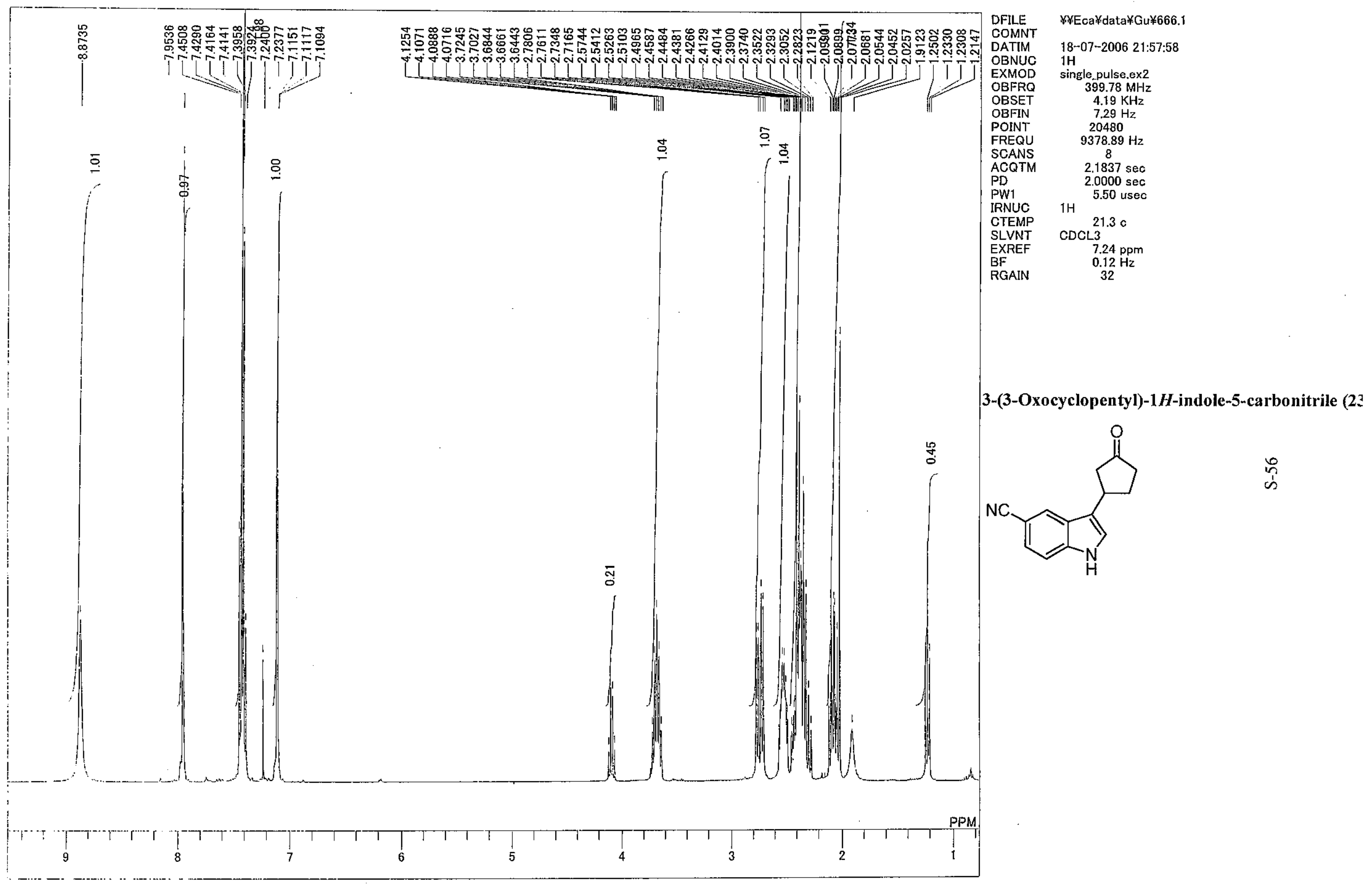


\#\#Eca¥data $¥ G u ¥ 666-13 C .1$

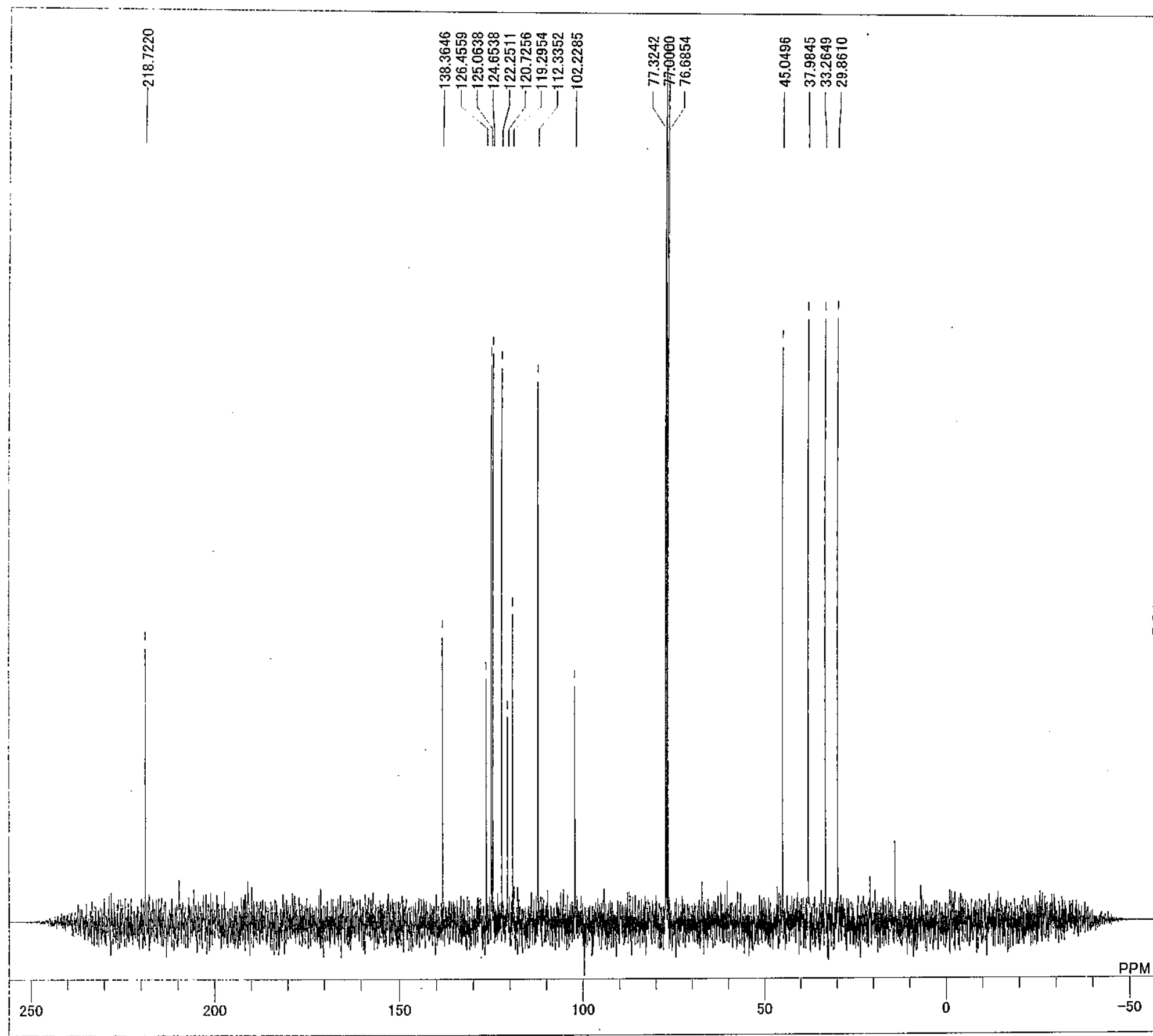

DFILE

OBSET

OBFIN

POINT

FREQU

SCANS

ACQTM

PD 1

PNUC

CTEMP

SLVNT

BF

$\operatorname{CDCL}^{21.8}$

$77.00 \mathrm{ppm}$

54

3-(3-Oxocyclopentyl)-1 $H$-indole-5-carbonitrile ( 23<smiles>N#Cc1ccc2[nH]cc(C3CCC(=O)C3)c2c1</smiles> 